Supporting Information

\title{
Total Synthesis of Sialyl Inositol Phosphosphingolipids CJP-2, CJP-3, and CJP-4 Isolated from Feather Star Comanthus japonica
}

Kenta Goto, Hideki Tamai, Yoh Takeda, Hide-Nori Tanaka, ${ }^{*}$ Takashi Mizuno, Akihiro Imamura, Hideharu Ishida, Makoto Kiso, Hiromune Ando*

E-mail: htanaka@gifu-u.ac.jp, hando@gifu-u.ac.jp

Table of Contents

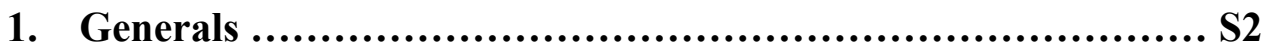

2. Experimental details ....................................... S3-S23

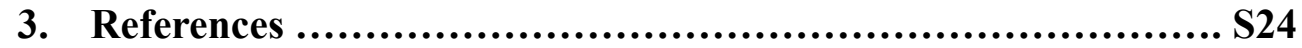

${ }^{1} \mathrm{H},{ }^{13} \mathrm{C}$, and ${ }^{31} \mathrm{P}$ NMR spectra of new compounds ............... S25-S90 


\section{Generals}

All reactions were carried out under a positive pressure of argon, unless otherwise noted. All chemicals were purchased from commercial suppliers and used without further purification, unless otherwise noted. Molecular sieves were purchased from Wako Pure Chemicals Industries, Ltd. and dried at $300{ }^{\circ} \mathrm{C}$ for $2 \mathrm{~h}$ in a muffle furnace prior to use. Solvents as reaction media were dried over molecular sieves and used without purification. TLC analysis was performed on Merck TLC plates (silica gel $60 \mathrm{~F}_{254}$ on glass plate) or Merck HPTLC plates (silica gel $60 \mathrm{~F}_{254}$ on glass plate). Compound detection was either by exposure to UV light ( $2536 \AA$ ) or by soak in a solution of $10 \% \mathrm{H}_{2} \mathrm{SO}_{4}$ solution in ethanol followed by heating. Silica gel ( 80 mesh, 300 mesh, and PSQ-60B) manufactured by Fuji Silysia Chemical, Ltd. was used for flash column chromatography. Quantity of silica gel was usually estimated as 100 to 200 -fold weight of sample to be charged. Solvent systems in chromatography were specified in $v / v$. Evaporation and concentration were carried out in vacuo. ${ }^{1} \mathrm{H},{ }^{13} \mathrm{C}$, and ${ }^{31} \mathrm{P} \mathrm{NMR}$ spectra were recorded with Bruker AVANCE III 500 spectrometer. Chemical shifts are expressed in ppm ( $\delta$ ) relative to the signal of $\mathrm{Me}_{4} \mathrm{Si}$ as an internal standard (for ${ }^{1} \mathrm{H}$ and ${ }^{13} \mathrm{C}$ NMR spectra) or $85 \%$ $\mathrm{H}_{3} \mathrm{PO}_{4}$ in $\mathrm{D}_{2} \mathrm{O}$ as an external standard (for ${ }^{31} \mathrm{P}$ NMR spectra), adjusted to $\delta 0.00 \mathrm{ppm}$, unless otherwise noted data are presented as follow: chemical shift, multiplicity $(\mathrm{s}=$ singlet, $\mathrm{d}=$ doublet, $\mathrm{t}=$ triplet, $\mathrm{dd}$ $=$ double of doublet, $\mathrm{td}=$ triple doublet, $\mathrm{m}=$ multiplet and/or multiple resonances), integration, coupling constant in hertz $(\mathrm{Hz})$, position of the corresponding proton. COSY methods were used to confirm the NMR signal assignments. High-resolution mass (ESI-TOF MS) spectra were run in the Bruker micrOTOF. Optical rotations were measured with a "Horiba SEPA-300" high-sensitive polarimeter. 


\section{Experimental details}

Methyl [phenyl 4,7,8-tri- $O$-acetyl-9-O-chloroacetyl-5-(2,2,2-trichloroethoxycarbamoyl)-3,5dideoxy-2-thio-D-glycero- $\beta$-D-galacto-2-nonulopyranosid]onate (13)

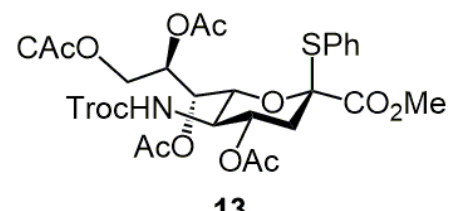

13

To a solution of methyl [phenyl-5-(2,2,2-trichloroethoxycarbamoyl)-3,5-dideoxy-2-thio-D-glycero- $\beta$ D-galacto-2-nonulopyranosid]onate $\mathbf{1}^{1}(10.0 \mathrm{~g}, 18.2 \mathrm{mmol})$ in THF $(364 \mathrm{~mL})$ were added chloroacetyl chloride (4.34 mL, $54.6 \mathrm{mmol})$ and 2,6-lutidine $(12.7 \mathrm{~mL}, 109 \mathrm{mmol})$ at $-80^{\circ} \mathrm{C}$. After stirring for $2 \mathrm{~h}$ at the same temperature, the completion of the reaction was confirmed by TLC $\left(10: 1 \mathrm{CHCl}_{3}-\mathrm{MeOH}\right)$. The reaction mixture was quenched with $\mathrm{MeOH}$ and then diluted with EtOAc. The resulting solution was washed with $2 \mathrm{M}$ aq. $\mathrm{HCl}, \mathrm{H}_{2} \mathrm{O}$, satd. aq. $\mathrm{NaHCO}_{3}$, and brine. The organic layer was successively dried over $\mathrm{Na}_{2} \mathrm{SO}_{4}$, concentrated, and exposed to high vacuum for $6 \mathrm{~h}$. The residue was then dissolved in THF $(91.0 \mathrm{~mL})$. Acetic anhydride $(10.2 \mathrm{~mL}, 109 \mathrm{mmol})$ and DMAP $(222 \mathrm{mg}, 1.82 \mathrm{mmol})$ were added to the mixture at $\mathrm{rt}$. After stirring for $1 \mathrm{~h}$ at the same temperature, the completion of the reaction was confirmed by TLC (1:1 n-hexane-EtOAc). The reaction mixture was quenched with $\mathrm{MeOH}$ and then diluted with EtOAc. The resulting solution was washed with $2 \mathrm{M}$ aq. $\mathrm{HCl}, \mathrm{H}_{2} \mathrm{O}$, satd. aq. $\mathrm{NaHCO}_{3}$, and brine. The organic layer was dried over $\mathrm{Na}_{2} \mathrm{SO}_{4}$ and concentrated. The resulting residue was purified by silica gel column chromatography (7:3 n-hexane-EtOAc) and then solidified from $(n$ hexane-EtOAc) to give $13(12.3 \mathrm{~g}, 90 \%)$ as white powder; $[\alpha]_{\mathrm{D}}-110.3{ }^{\circ}\left(\mathrm{c} 1.0, \mathrm{CDCl}_{3}\right) ;{ }^{1} \mathrm{H} \mathrm{NMR}$ $\left(500 \mathrm{MHz}, \mathrm{CDCl}_{3}\right) \delta 7.46-7.33(\mathrm{~m}, 5 \mathrm{H}, \mathrm{Ph}), 5.52\left(\mathrm{t}, 1 \mathrm{H}, J_{6,7}=J_{7,8}=2.0 \mathrm{~Hz}, \mathrm{H}-7\right), 5.46\left(\mathrm{td}, 1 \mathrm{H}, J_{3 \mathrm{eq}, 4}\right.$ $\left.=4.5 \mathrm{~Hz}, J_{3 \mathrm{ax}, 4}=J_{4,5}=11.0 \mathrm{~Hz}, \mathrm{H}-4\right), 5.23\left(\mathrm{~d}, 1 \mathrm{H}, J_{5, \mathrm{NH}}=10.0 \mathrm{~Hz}, \mathrm{NH}\right) 5.01\left(\mathrm{dt}, 1 \mathrm{H}, J_{8,9 \mathrm{a}}=2.5 \mathrm{~Hz}\right.$, $\left.J_{8,9 \mathrm{~b}}=8.5 \mathrm{~Hz}, \mathrm{H}-8\right), 4.91\left(\mathrm{~d}, 1 \mathrm{H}, J_{\mathrm{gem}}=12.0 \mathrm{~Hz}, \mathrm{CCl}_{3} \mathrm{CH}_{2} \mathrm{O}\right), 4.70\left(\mathrm{dd}, 1 \mathrm{H}, J_{5,6}=10.5 \mathrm{~Hz}, \mathrm{H}-6\right), 4.58$ $\left(\mathrm{dd}, 1 \mathrm{H}, J_{\mathrm{gem}}=12.0 \mathrm{~Hz}, \mathrm{H}-9 \mathrm{a}\right), 4.49\left(\mathrm{~d}, 1 \mathrm{H}, \mathrm{CCl}_{3} \mathrm{CH}_{2} \mathrm{O}\right), 4.14(\mathrm{dd}, 1 \mathrm{H}, \mathrm{H}-9 \mathrm{~b}), 3.98\left(\mathrm{~d}, 1 \mathrm{H}, J_{\mathrm{gem}}=\right.$ $14.5 \mathrm{~Hz}, \mathrm{ClCH}_{2} \mathrm{CO}$ ), 3.93 (d, $1 \mathrm{H}, \mathrm{ClCH}_{2} \mathrm{CO}$ ), 3.80 (q, $1 \mathrm{H}, \mathrm{H}-5$ ), 3.63 (s, $3 \mathrm{H}, \mathrm{OMe}$ ), 2.74 (dd, $1 \mathrm{H}$, $\left.J_{\text {gem }}=15.0 \mathrm{~Hz}, \mathrm{H}-3 \mathrm{eq}\right), 2.15-2.01(\mathrm{~m}, 10 \mathrm{H}, \mathrm{H}-3 \mathrm{ax}, 3 \mathrm{Ac}) ;{ }^{13} \mathrm{C} \mathrm{NMR}\left(125 \mathrm{MHz}, \mathrm{CDCl}_{3}\right) \delta 170.9$, 170.4, 170.0, 168.2, 166.6, 154.3, 136.2, 129.9, 129.2, 128.7, 95.4, 88.7, 74.6, 72.9, 72.5, 68.9, 68.6, 64.0, 52.7, 51.7, 40.6, 37.5, 31.6, 22.6, 20.9, 20.8, 20.7; HRMS (ESI) $\mathrm{m} / z$ : found $[\mathrm{M}+\mathrm{Na}]^{+} 772.0161$, $\mathrm{C}_{27} \mathrm{H}_{31} \mathrm{Cl}_{4} \mathrm{NO}_{13} \mathrm{~S}$ calcd for $[\mathrm{M}+\mathrm{Na}]^{+} 772.0162$.

Methyl [phenyl 4,7,8-tri- $O$-acetyl-5-(2,2,2-trichloroehoxycarbamoyl)-3,5-dideoxy-9-O-methyl-2thio-D-glycero- $\beta$-D-galacto-2-nonulopyranosid]onate (15) 


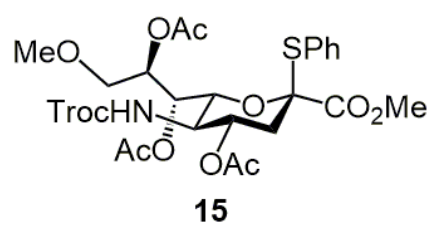

To a solution of $\mathbf{1 3}(303 \mathrm{mg}, 403 \mu \mathrm{mol})$ in DMF $(8.00 \mathrm{~mL})$ were added 1-selenocarbamoylpiperidine $\mathbf{1 4}^{2}(116 \mathrm{mg}, 607 \mu \mathrm{mol})$ and 2,6-lutidine $(94 \mu \mathrm{L}, 816 \mu \mathrm{mol})$ at rt. After stirring for $1 \mathrm{~h}$ at $65^{\circ} \mathrm{C}$, the completion of the reaction was confirmed by TLC (2:3n-hexane-EtOAc). The reaction mixture was cooled to rt and then diluted with EtOAc. The resulting solution was washed with $2 \mathrm{M}$ aq. $\mathrm{HCl}, \mathrm{H}_{2} \mathrm{O}$, satd. aq. $\mathrm{NaHCO}_{3}$, and brine. The organic layer was successively dried over $\mathrm{Na}_{2} \mathrm{SO}_{4}$, concentrated, and exposed to high vacuum for $3 \mathrm{~h}$. The residue was then dissolved in $\mathrm{CH}_{2} \mathrm{Cl}_{2}(40.0 \mathrm{~mL})$. MeOTf $(310 \mu \mathrm{L}, 2.74 \mathrm{mmol})$ and DTBMP $(1.17 \mathrm{~g}, 5.64 \mathrm{mmol})$ were added to the mixture at rt. After stirring for 5 days at $30^{\circ} \mathrm{C}$, the completion of the reaction was confirmed by TLC ( $2: 3 n$-hexane-EtOAc). The reaction mixture was quenched with satd. aq. $\mathrm{NaHCO}_{3}$ and then extracted with $\mathrm{CHCl}_{3}$. The organic layer was successively washed with satd. aq. $\mathrm{NaHCO}_{3}, \mathrm{H}_{2} \mathrm{O}$, and brine, dried over $\mathrm{Na}_{2} \mathrm{SO}_{4}$, and concentrated. The resulting residue was purified by silica gel column chromatography (5:2 $n$-hexaneEtOAc) to give 15 (214 mg, 77\%) as white foamy material; $[\alpha]_{\mathrm{D}}-81.0^{\circ}\left(\mathrm{c} 1.2, \mathrm{CDCl}_{3}\right) ;{ }^{1} \mathrm{H} \mathrm{NMR}(500$ $\left.\mathrm{MHz}, \mathrm{CDCl}_{3}\right) \delta 7.48-7.31(\mathrm{~m}, 5 \mathrm{H}, \mathrm{Ph}), 5.52\left(\mathrm{t}, 1 \mathrm{H}, J_{6,7}=J_{7,8}=2.0 \mathrm{~Hz}, \mathrm{H}-7\right), 5.44\left(\mathrm{td}, 1 \mathrm{H}, J_{3 \mathrm{eq}, 4}=\right.$ $\left.5.0 \mathrm{~Hz}, J_{3 \mathrm{ax}, 4}=J_{4,5}=11.0 \mathrm{~Hz}, \mathrm{H}-4\right), 5.24\left(\mathrm{~d}, 1 \mathrm{H}, J_{5, \mathrm{NH}}=10.0 \mathrm{~Hz}, \mathrm{NH}\right), 5.01\left(\mathrm{td}, 1 \mathrm{H}, J_{8,9 \mathrm{a}}=2.5 \mathrm{~Hz}\right.$, $\left.J_{8,9 \mathrm{~b}}=8.0 \mathrm{~Hz}, \mathrm{H}-8\right), 4.90\left(\mathrm{~d}, 1 \mathrm{H}, J_{\mathrm{gem}}=12.0 \mathrm{~Hz}, \mathrm{CCl}_{3} \mathrm{CH}_{2} \mathrm{O}\right.$ ), $4.71\left(\mathrm{dd}, 1 \mathrm{H}, J_{5,6}=10.5 \mathrm{~Hz}, \mathrm{H}-6\right), 4.50$ (d, $1 \mathrm{H}, \mathrm{CCl}_{3} \mathrm{CH}_{2} \mathrm{O}$ ), 3.84-3.76 (m, $\left.2 \mathrm{H}, \mathrm{H}-5, \mathrm{H}-9 \mathrm{a}\right), 3.62$ (s, $\left.3 \mathrm{H}, \mathrm{OMe}\right), 3.43$ (dd, $1 \mathrm{H}, J_{\mathrm{gem}}=11.0$ Hz, H-9b), 3.11 (s, 3 H, OMe), 2.76 (dd, 1 H, $J_{\text {gem }}=13.5$ Hz, H-3eq), 2.13-2.01 (m, 10 H, H-3ax, 3 Ac); ${ }^{13} \mathrm{C}$ NMR $\left(125 \mathrm{MHz}, \mathrm{CDCl}_{3}\right) \delta 171.3,170.4,170.1,167.9,154.3,136.0,129.8,129.2,128.9$, 95.4, 89.0, 77.6, 74.5, 73.6, 73.1, 70.1, 69.4, 68.8, 58.7, 52.5, 51.8, 37.7, 21.2, 20.8, 20.7; HRMS (ESI) $m / z$ : found $[\mathrm{M}+\mathrm{Na}]^{+} 710.0604, \mathrm{C}_{26} \mathrm{H}_{32} \mathrm{Cl}_{3} \mathrm{NO}_{12} \mathrm{~S}$ calcd for $[\mathrm{M}+\mathrm{Na}]^{+} 710.0603$.

Benzyl [methyl 4,7,8-tri- $O$-acetyl-5-(2,2,2-trichloroethoxycarbamoyl)-3,5-dideoxy-9-O-methyl-Dglycero-D-galacto-2-nonulopyranosylonat]oxyacetate (16)

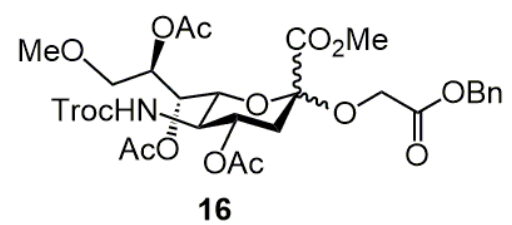

A solution of 15 (1.00 g, $1.45 \mathrm{mmol})$ and benzyl glycolate $9(309 \mu \mathrm{L}, 2.18 \mathrm{mmol})$ in EtCN (14.5 mL) was transferred into a two-neck round bottom flask, in which $3 \AA$ molecular sieves (725 mg) were placed, using a cannula, followed by addition of $N$-iodosuccinimide (490 mg, $2.18 \mathrm{mmol}$ ). The suspension was stirred for $30 \mathrm{~min}$ at $\mathrm{rt}$ and then cooled to $-80{ }^{\circ} \mathrm{C}$. Subsequently, triethyl trifluoromethanesulfonate ( $38 \mu \mathrm{L}, 435 \mu \mathrm{mol}$ ) was added to the mixture. After stirring for $5 \mathrm{~h}$ at the 
same temperature, the completion of the reaction was confirmed by TLC ( $7: 1 \mathrm{CHCl}_{3}$-acetone). The reaction mixture was quenched with satd. aq. $\mathrm{NaHCO}_{3}$ and then filtered through a pad of Celite, washed with $\mathrm{CHCl}_{3}$. The combined filtrate and washings were successively washed with satd. aq. $\mathrm{Na}_{2} \mathrm{~S}_{2} \mathrm{O}_{3}$ and brine, dried over $\mathrm{Na}_{2} \mathrm{SO}_{4}$, and concentrated. The resulting residue was purified by silica gel column chromatography (10:3:1 $n$-hexane- $\left.\mathrm{CHCl}_{3}-\mathrm{MeOH}\right)$ to give $\mathbf{1 6} \boldsymbol{\alpha}(860 \mathrm{mg}, 79 \%)$ as white foamy material along with $\mathbf{1 6 \beta}(7 \%)$.

16 $:[\alpha]_{\mathrm{D}}+2.5^{\circ}\left(\mathrm{c} 1.0, \mathrm{CHCl}_{3}\right) ;{ }^{1} \mathrm{H}$ NMR $\left(500 \mathrm{MHz}, \mathrm{CDCl}_{3}\right) \delta 7.40-7.30(\mathrm{~m}, 5 \mathrm{H}, \mathrm{Ph}), 5.38(\mathrm{dd}, 1 \mathrm{H}$, $\left.J_{6,7}=2.0 \mathrm{~Hz}, J_{7,8}=7.5 \mathrm{~Hz}, \mathrm{H}-7\right), 5.33(\mathrm{~m}, 1 \mathrm{H}, \mathrm{H}-8), 5.20\left(\mathrm{~d}, 1 \mathrm{H}, J_{\mathrm{gem}}=12.0 \mathrm{~Hz}, \mathrm{OCH}_{2} \mathrm{CO}\right), 5.16(\mathrm{~d}$, $\left.1 \mathrm{H}, \mathrm{OCH}_{2} \mathrm{CO}\right), 5.01\left(\mathrm{td}, 1 \mathrm{H}, J_{3 \mathrm{eq}, 4}=5.0 \mathrm{~Hz}, J_{3 \mathrm{ax}, 4}=J_{4,5}=10.5 \mathrm{~Hz}, \mathrm{H}-4\right), 4.38\left(\mathrm{~m}, 2 \mathrm{H}, \mathrm{NH}, \mathrm{PhCH}_{2}\right)$, $4.49\left(\mathrm{~d}, 1 \mathrm{H}, J_{\text {gem }}=12.0 \mathrm{~Hz}, \mathrm{PhCH}_{2}\right), 4.39\left(\mathrm{~d}, 1 \mathrm{H}, J_{\text {gem }}=16.5 \mathrm{~Hz}, \mathrm{CCl}_{3} \mathrm{CH}_{2} \mathrm{O}\right), 4.28(\mathrm{~d}, 1 \mathrm{H}$, $\mathrm{CCl}_{3} \mathrm{CH}_{2} \mathrm{O}$ ), 4.03 (dd, $\left.1 \mathrm{H}, J_{5,6}=10.5 \mathrm{~Hz}, \mathrm{H}-6\right), 3.74$ (s, $3 \mathrm{H}, \mathrm{OMe}$ ), 3.50 (q, $1 \mathrm{H}, J_{5, \mathrm{NH}}=10.5 \mathrm{~Hz}, \mathrm{H}-$ 5), 3.54 (dd, $\left.1 \mathrm{H}, J_{8,9 \mathrm{a}}=3.5 \mathrm{~Hz}, J_{\text {gem }}=11.0 \mathrm{~Hz}, \mathrm{H}-9 \mathrm{a}\right), 3.38$ (dd, $\left.1 \mathrm{H}, J_{8,9 \mathrm{~b}}=5.0 \mathrm{~Hz}, \mathrm{H}-9 \mathrm{~b}\right), 3.29$ (s, 3 $\mathrm{H}, \mathrm{OMe}$ ), 2.78 (dd, $\left.1 \mathrm{H}, J_{\text {gem }}=13.0 \mathrm{~Hz}, \mathrm{H}-3 \mathrm{eq}\right), 2.13-2.01$ (3 s, $\left.9 \mathrm{H}, 3 \mathrm{Ac}\right), 1.96$ (t, $\left.1 \mathrm{H}, \mathrm{H}-3 \mathrm{ax}\right) ;{ }^{13} \mathrm{C}$ NMR (125 MHz, $\left.\mathrm{CDCl}_{3}\right) \delta 170.4,170.0,169.1,167.4,154.0,135.4,128.5,128.3,98.0,95.3,74.5$, $72.3,71.1,68.5,68.467 .8,66.6,61.9,59.1,52.9,51.5,37.7,21.2,20.8$; Gated $\left\{{ }^{1} \mathrm{H}\right\}{ }^{13} \mathrm{C}$ NMR $(125$ $\left.\mathrm{MHz}, \mathrm{CDCl}_{3}\right) \delta 167.8\left(J_{\mathrm{Cl} 1 \mathrm{H} 3 \mathrm{ax}}=6.3 \mathrm{~Hz}, \mathrm{C}-1\right)$; HRMS (ESI) $m / z$ : found $[\mathrm{M}+\mathrm{Na}]^{+} 766.1045$, $\mathrm{C}_{29} \mathrm{H}_{36} \mathrm{Cl}_{3} \mathrm{NO}_{15}$ calcd for $[\mathrm{M}+\mathrm{Na}]^{+} 766.1043$.

16及: $[\alpha]_{\mathrm{D}}-1.3^{\circ}\left(\mathrm{c} 1.6, \mathrm{CHCl}_{3}\right) ;{ }^{1} \mathrm{H}$ NMR (500 MHz, $\left.\mathrm{CDCl}_{3}\right) \delta 7.41-7.37(\mathrm{~m}, 5 \mathrm{H}, \mathrm{Ph}), 5.30-5.20(\mathrm{~m}$, $\left.4 \mathrm{H}, \mathrm{H}-4, \mathrm{H}-7, \mathrm{H}-8, \mathrm{OCH}_{2} \mathrm{CO}\right), 5.15\left(\mathrm{~d}, 1 \mathrm{H}, J_{\mathrm{gem}}=12.0 \mathrm{~Hz}, \mathrm{OCH}_{2} \mathrm{CO}\right), 4.90\left(\mathrm{~d}, 1 \mathrm{H}, J_{\mathrm{gem}}=12.1 \mathrm{~Hz}\right.$, $\left.\mathrm{PhCH}_{2}\right), 4.68\left(\mathrm{~d}, 1 \mathrm{H}, J_{5, \mathrm{NH}}=10.3 \mathrm{~Hz}, \mathrm{NH}\right), 4.48\left(\mathrm{~d}, 1 \mathrm{H}, \mathrm{PhCH} H_{2}\right), 4.29\left(\mathrm{~d}, 1 \mathrm{H}, J_{\mathrm{gem}}=15.7 \mathrm{~Hz}\right.$, $\left.\mathrm{CCl}_{3} \mathrm{CH}_{2} \mathrm{O}\right), 4.21\left(\mathrm{~d}, 1 \mathrm{H}, \mathrm{CCl}_{3} \mathrm{CH}_{2} \mathrm{O}\right), 4.09\left(\mathrm{dd}, 1 \mathrm{H}, J_{6,7}=1.8 \mathrm{~Hz}, J_{5,6}=10.6 \mathrm{~Hz}, \mathrm{H}-6\right), 3.80-3.71(\mathrm{~m}$, 5 H, H-5, H-9a, OMe), 3.44 (dd, 1 H, $J_{8,9 b}=6.7$ Hz, $J_{\text {gem }}=11.0$ Hz, H-9b), 3.30 (s, 3 H, OMe), 2.57 $\left(\mathrm{dd}, 1 \mathrm{H}, J_{3 \mathrm{eq}, 4}=4.9 \mathrm{~Hz}, J_{\mathrm{gem}}=13.1 \mathrm{~Hz}, \mathrm{H}-3 \mathrm{eq}\right), 2.13,2.05$ and $2.00(3 \mathrm{~s}, 9 \mathrm{H}, 3 \mathrm{Ac}), 1.95$ (dd, $J_{3 \mathrm{ax}, 4}=$ $11.7 \mathrm{~Hz}, \mathrm{H}-3 \mathrm{ax}) ;{ }^{13} \mathrm{C} \mathrm{NMR}\left(125 \mathrm{MHz}, \mathrm{CDCl}_{3}\right) \delta 170.5,170.3,170.0,168.9,166.6,154.3,135.1,128.8$, 128.7, 128.7, 98.1, 95.4, 74.6, 71.9, 71.5, 71.0, 68.7, 68.3, 67.1, 61.3, 59.0, 52.8, 51.5, 36.9, 29.7, 21.0, 20.8, 20.7; HRMS (ESI) $m / z$ : found $[\mathrm{M}+\mathrm{Na}]^{+} 766.1045, \mathrm{C}_{29} \mathrm{H}_{36} \mathrm{Cl}_{3} \mathrm{NO}_{15}$ calcd for $[\mathrm{M}+\mathrm{Na}]^{+} 766.1043$.

Benzyl (methyl 5-acetoxyacetamido-4,7,8-tri-O-acetyl-3,5-dideoxy-9-O-methyl-D-glycero- $\alpha$-Dgalacto-2-nonulopyranosylonat)oxyacetate (17)

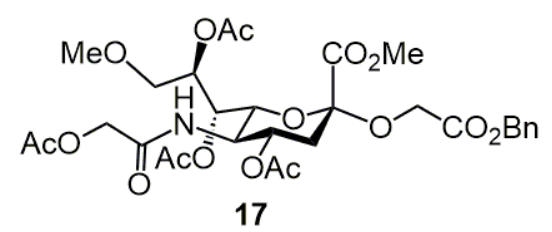

To a solution of $\mathbf{1 6} \boldsymbol{\alpha}(75 \mathrm{mg}, 100 \mu \mathrm{mol})$ in $\mathrm{MeCN}-\mathrm{AcOH}(4: 1,2.4 \mathrm{~mL})$ was added $\mathrm{Zn}$ powder $(375$ $\mathrm{mg}$, excess) at rt. After stirring for $30 \mathrm{~min}$ at $\mathrm{rt}$, the completion of the reaction was confirmed by TLC $\left(15: 1 \mathrm{CHCl}_{3}-\mathrm{MeOH}\right)$. The reaction mixture was filtered through a pad of Celite, washed with $\mathrm{CHCl}_{3}$. 
The combined filtrate and washings were successively washed with satd. aq. $\mathrm{NaHCO}_{3}$ and brine, dried over $\mathrm{Na}_{2} \mathrm{SO}_{4}$, concentrated, and exposed to high vacuum for $3 \mathrm{~h}$. The residue was then dissolved in THF $(2.0 \mathrm{~mL})$. Acetoxyacetyl chloride $(16 \mu \mathrm{L}, 150 \mu \mathrm{mol})$ and triethylamine $(70 \mu \mathrm{L}, 500 \mu \mathrm{mol})$ were added to the mixture at $0{ }^{\circ} \mathrm{C}$. After stirring for $10 \mathrm{~min}$ at the same temperature, the completion of the reaction was confirmed by TLC (2:1 toluene-acetone). The reaction mixture was diluted with $\mathrm{CHCl}_{3}$. The organic layer was successively washed with $\mathrm{H}_{2} \mathrm{O}$, dried over $\mathrm{Na}_{2} \mathrm{SO}_{4}$, and concentrated. The resulting residue was purified by silica gel column chromatography (4:1 toluene-acetone) to give $\mathbf{1 7}$ (64 mg, 95\%) as white foamy material; $[\alpha]_{\mathrm{D}}-13.5^{\circ}\left(\mathrm{c} 0.4, \mathrm{CHCl}_{3}\right) ;{ }^{1} \mathrm{H} \mathrm{NMR}\left(500 \mathrm{MHz}, \mathrm{CDCl}_{3}\right) \delta$ 7.39-7.31 (m, 5 H, Ph), 5.94 (d, 1 H, $\left.J_{5, \mathrm{NH}}=9.5 \mathrm{~Hz}, \mathrm{NH}\right), 5.34-5.27$ (m, $\left.2 \mathrm{H}, \mathrm{H}-7, \mathrm{H}-8\right), 5.21$ (d, $1 \mathrm{H}$, $J_{\mathrm{gem}}=12.5 \mathrm{~Hz}, \mathrm{PhCH}_{2}$ ), 5.15 (d, $1 \mathrm{H}, \mathrm{PhCH}_{2}$ ), 4.99 (m, $\left.1 \mathrm{H}, \mathrm{H}-4\right), 4.60-4.26$ (m, $4 \mathrm{H}, \mathrm{H}-11 \mathrm{a}, \mathrm{H}-11 \mathrm{~b}$, $\mathrm{OCH}_{2} \mathrm{CO}$ ), 4.10 (q, $\left.1 \mathrm{H}, J_{4,5}=J_{5,6}=10.5 \mathrm{~Hz}, \mathrm{H}-5\right), 4.03\left(\mathrm{dd}, 1 \mathrm{H}, J_{6,7}=2.0 \mathrm{~Hz}, \mathrm{H}-6\right), 3.75(\mathrm{~s}, 3 \mathrm{H}$, OMe), $3.56\left(\mathrm{dd}, 1 \mathrm{H}, J_{8,9 \mathrm{a}}=2.5 \mathrm{~Hz}, J_{\text {gem }}=10.5 \mathrm{~Hz}, \mathrm{H}-9 \mathrm{a}\right), 3.34$ (dd, $\left.1 \mathrm{H}, J_{8,9 \mathrm{~b}}=5.0 \mathrm{~Hz}, \mathrm{H}-9 \mathrm{~b}\right), 3.29$ (s, $3 \mathrm{H}, \mathrm{OMe}$ ), 2.74 (dd, $\left.1 \mathrm{H}, J_{\text {3eq, } 4}=4.5 \mathrm{~Hz}, \mathrm{H}-3 \mathrm{eq}\right), 2.19-1.99$ (m, $13 \mathrm{H}, \mathrm{H}-3 \mathrm{ax}, 4 \mathrm{Ac}$ ); ${ }^{13} \mathrm{C} \mathrm{NMR}$ $\left(125 \mathrm{MHz}, \mathrm{CDCl}_{3}\right) \delta 171.0,170.1,170.1,169.6,169.1$ 167.6, 135.3, 129.9, 129.6, 128.6, 128.5, 128.4, $128.3,128.1,98.1,77.3,72.6,70.0,68.9,68.2,67.6,66.6,62.7,61.8,59.1,52.9,49.2,37.6,35.8,31.8$, 29.7, 29.6, 29.6, 29.5, 29.4, 29.3, 29.2, 29.2, 29.0, 27.1, 27.1, 25.5, 22.6, 21.2, 20.8, 20.7, 20.6, 14.0; HRMS (ESI) $m / z$ : found $[\mathrm{M}+\mathrm{Na}]^{+} 692.2161, \mathrm{C}_{30} \mathrm{H}_{39} \mathrm{NO}_{16}$ calcd for $[\mathrm{M}+\mathrm{Na}]^{+} 692.2162$.

(Methyl 5-acetoxyacetamido-4,7,8-tri-O-acetyl-3,5-dideoxy-9-O-methyl-D-glycero- $\alpha$-D-galacto-2nonulopyranosylonat)oxyacetic acid (18)

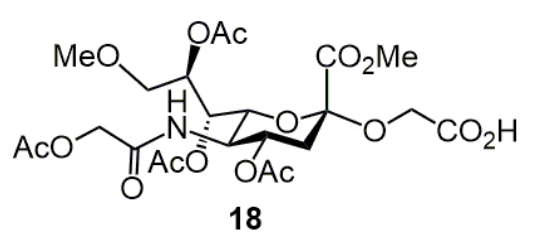

To a solution of 17 (334 mg, $499 \mu \mathrm{mol})$ in EtOAc $(10.0 \mathrm{~mL})$ was added palladium hydroxide on carbon (20\% wt. loading; $167 \mathrm{mg}$ ) at rt. After stirring for $15 \mathrm{~min}$ at $\mathrm{rt}$ under a hydrogen atmosphere, the completion of the reaction was confirmed by TLC $\left(10: 1 \mathrm{CHCl}_{3}-\mathrm{MeOH}\right)$. The reaction mixture was filtered through a pad of Celite, washed with EtOAc. The combined filtrate and washings were concentrated to give 18 (290 mg, quant.) as white foamy material; $[\alpha]_{\mathrm{D}}-14.2{ }^{\circ}\left(\mathrm{c} 0.5, \mathrm{CHCl}_{3}\right) ;{ }^{1} \mathrm{H}$ NMR (500 MHz, $\left.\mathrm{CDCl}_{3}\right) \delta 6.06\left(\mathrm{~d}, 1 \mathrm{H}, J_{5, \mathrm{NH}}=7.5 \mathrm{~Hz}, \mathrm{NH}\right), 5.35-5.29(\mathrm{~m}, 2 \mathrm{H}, \mathrm{H}-7, \mathrm{H}-8), 5.02(\mathrm{~m}$, $1 \mathrm{H}, \mathrm{H}-4), 4.60-4.22$ (m, 4 H, H-11a, H-11b, OCH $\mathrm{C}_{2} \mathrm{CO}$ ), 4.15-4.07 (m, 2 H, H-5, H-6), 3.83 (s, 3 H, OMe), 3.57 (dd, $\left.1 \mathrm{H}, J_{8,9 \mathrm{a}}=2.5 \mathrm{~Hz}, J_{\text {gem }}=9.5 \mathrm{~Hz}, \mathrm{H}-9 \mathrm{a}\right), 3.37$ (dd, $\left.1 \mathrm{H}, J_{8,9 \mathrm{~b}}=4.5 \mathrm{~Hz}, \mathrm{H}-9 \mathrm{~b}\right), 3.31$ (s, $3 \mathrm{H}, \mathrm{OMe}$ ), 2.72 (dd, $\left.1 \mathrm{H}, J_{3 \mathrm{eq}, 4}=4.5 \mathrm{~Hz}, J_{\mathrm{gem}}=12.5 \mathrm{~Hz}, \mathrm{H}-3 \mathrm{eq}\right), 2.10-2.00$ (m, 13, H, H-3ax, $4 \mathrm{Ac}$ ); ${ }^{13} \mathrm{C}$ NMR $\left(125 \mathrm{MHz}, \mathrm{CDCl}_{3}\right) \delta 170.2,171.3,171.1,170.5,170.2,169.7,167.9,167.7,98.2$, 72.6, 71.0, 69.0, 68.2, 67.6, 62.8, 61.7, 60.4, 59.1, 53.1, 49.3, 37.5, 31.9, 29.7, 29.6, 29.6, $29.4,29.3,29.3,29.2,25.5,22.6,21.2,21.0,20.8,20.7,20.6,14.1$; HRMS (ESI) $\mathrm{m} / \mathrm{z}$ : found 
$[\mathrm{M}-\mathrm{H}]^{-} 578.1726, \mathrm{C}_{23} \mathrm{H}_{32} \mathrm{NO}_{16}$ calcd for $[\mathrm{M}-\mathrm{H}]^{-} 578.1727$.

Benzyl [methyl 4,7,8-tri- $O$-acetyl-3,5-dideoxy-9- $O$-methyl-5-(methyl 5-acetoxyacetamido-4,7,8-tri$O$-acetyl-3,5-dideoxy-9-O-methyl-D-glycero- $\alpha$-D-galacto-2-nonulopyranosylonat)oxyacetamido-Dglycero- $\alpha$-D-galacto-2-nonulopyranosylonat]oxyacetate (19)

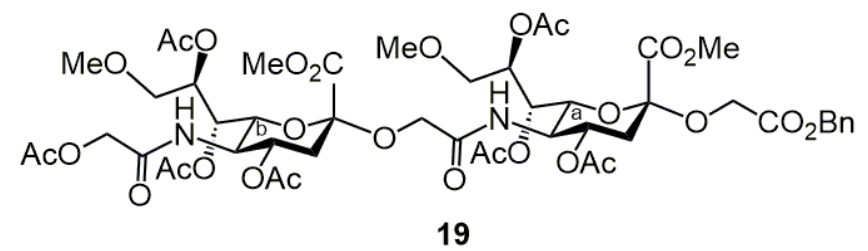

To a solution of $16(100 \mathrm{mg}, 134 \mu \mathrm{mol})$ in $\mathrm{MeCN}-\mathrm{AcOH}(4: 1,2.7 \mathrm{~mL})$ was added $\mathrm{Zn}$ powder (500 $\mathrm{mg}$, excess) at rt. After stirring for $30 \mathrm{~min}$ at $\mathrm{rt}$, the completion of the reaction was confirmed by TLC (20:1 $\left.\mathrm{CHCl}_{3}-\mathrm{MeOH}\right)$. The reaction mixture was filtered through a pad of Celite, washed with $\mathrm{CHCl}_{3}$. The combined filtrate and washings were successively washed with satd. aq. $\mathrm{NaHCO}_{3}$ and brine, dried over $\mathrm{Na}_{2} \mathrm{SO}_{4}$, concentrated, and exposed to high vacuum for $1 \mathrm{~h}$. The residue was then dissolved in DMF (2.7 mL). 18 (78 mg, $135 \mu \mathrm{mol})$ and HBTU (62 mg, $161 \mu \mathrm{mol})$ were added to the mixture at rt. The mixture was stirred for $2 \mathrm{~h}$ at the same temperature before addition of DIEA ( $37 \mu \mathrm{L}, 201 \mu \mathrm{mol})$. After stirring for additional $30 \mathrm{~min}$, the completion of the reaction was confirmed by TLC (20:1 $\mathrm{CHCl}_{3}-\mathrm{MeOH}$ ). The reaction mixture was diluted with EtOAc. The organic layer was successively washed with $\mathrm{H}_{2} \mathrm{O}$ and brine, dried over $\mathrm{Na}_{2} \mathrm{SO}_{4}$, and concentrated. The resulting residue was purified by silica gel column chromatography $\left(80: 1 \mathrm{CHCl}_{3}-\mathrm{MeOH}\right)$ to give $19(131 \mathrm{mg}, 86 \%)$ as a white amorphous solid; $[\alpha]_{\mathrm{D}}-8.7^{\circ}\left(\mathrm{c} 1.1, \mathrm{CHCl}_{3}\right) ;{ }^{1} \mathrm{H} \mathrm{NMR}\left(500 \mathrm{MHz}, \mathrm{CDCl}_{3}\right) \delta 7.40-7.30(\mathrm{~m}, 5 \mathrm{H}, \mathrm{Ph})$, 6.13-5.86 (2 d, 2 H, NH $\left.{ }^{a}, \mathrm{NH}^{b}\right), 5.35-5.23\left(\mathrm{~m}, 4 \mathrm{H}, \mathrm{H}-7^{a}, \mathrm{H}-8^{a}, \mathrm{H}-7^{b}, \mathrm{H}-8^{b}\right), 5.21\left(\mathrm{~d}, 1 \mathrm{H}, J_{\text {gem }}=12.0\right.$ $\left.\mathrm{Hz}, \mathrm{PhCH}_{2}\right), 5.15$ (d, $\left.1 \mathrm{H}, \mathrm{PhCH}_{2}\right), 5.01-4.94\left(\mathrm{~m}, 2 \mathrm{H}, \mathrm{H}-4^{a}, \mathrm{H}-4^{b}\right), 4.59$ (d, $1 \mathrm{H}, J_{\mathrm{gem}}=15.5 \mathrm{~Hz}, \mathrm{H}-$ $\left.11 \mathrm{a}^{b}\right), 4.39\left(\mathrm{~d}, 1 \mathrm{H}, J_{\mathrm{gem}}=16.0 \mathrm{~Hz}, \mathrm{OCH}_{2} \mathrm{CO}\right), 4.33-4.27\left(\mathrm{~m}, 2 \mathrm{H}, \mathrm{H}-11 \mathrm{~b}^{b}, \mathrm{OCH}_{2} \mathrm{CO}\right), 4.24(\mathrm{dd}, 1 \mathrm{H}$, $\left.J_{6,7}=2.0 \mathrm{~Hz}, J_{5,6}=11.0 \mathrm{~Hz}, \mathrm{H}-6^{b}\right), 4.20-4.09\left(\mathrm{~m}, 3 \mathrm{H}, \mathrm{H}-5^{a}, \mathrm{H}-11 \mathrm{a}^{a}, \mathrm{H}-5^{b}\right), 4.01\left(\mathrm{dd}, 1 \mathrm{H}, J_{6,7}=2.0\right.$ $\left.\mathrm{Hz}, J_{5,6}=11.0 \mathrm{~Hz}, \mathrm{H}-6^{a}\right), 3.88(\mathrm{~s}, 3 \mathrm{H}, \mathrm{OMe}), 3.80\left(\mathrm{~d}, 1 \mathrm{H}, J_{\mathrm{gem}}=15.0 \mathrm{~Hz}, \mathrm{H}-11 \mathrm{~b}^{a}\right), 3.76(\mathrm{~s}, 3 \mathrm{H}, \mathrm{OMe})$, 3.59-3.26 (m, 10 H, H-9a ${ }^{a}, \mathrm{H}_{-} 9 \mathrm{~b}^{a}, \mathrm{H}-9 \mathrm{a}^{b}, \mathrm{H}-9 \mathrm{~b}^{b}, 2 \mathrm{OMe}$ ), 2.72 (dd, $1 \mathrm{H}, J_{3 \mathrm{eq}, 4}=5.0 \mathrm{~Hz}, J_{\mathrm{gem}}=13.0$ $\left.\mathrm{Hz}, \mathrm{H}-3 \mathrm{eq}^{a}\right), 2.68\left(\mathrm{dd}, 1 \mathrm{H}, J_{3 \mathrm{eq}, 4}=9.5 \mathrm{~Hz}, J_{\mathrm{gem}}=12.5 \mathrm{~Hz}, \mathrm{H}-3 \mathrm{eq}^{b}\right), 2.22-2.00\left(\mathrm{~m}, 23 \mathrm{H}, \mathrm{H}-3 \mathrm{ax}^{a}, \mathrm{H}-\right.$ $\left.3 \mathrm{ax}^{b}, 7 \mathrm{Ac}\right) ;{ }^{13} \mathrm{C} \mathrm{NMR}\left(125 \mathrm{MHz}, \mathrm{CDCl}_{3}\right) \delta 171.0,170.5,170.1,170.1,170.0,170.0,169.6,169.2$, 168.6, 167.8, 167.6, 135.4, 128.6, 128.5, 128.5, 128.4, 128.3, 98.4, 98.1, 77.2, 73.1, 72.8, 71.0, 70.3, $69.1,68.8,68.6,68.1,67.6,67.6,66.9,66.6,63.9,62.8,61.9,59.3,59.2,59.1,53.3,52.9,49.2,48.8$, 37.7, 37.7, 21.3, 21.2, 20.9, 20.8, 20.8, 20.7, 20.7; HRMS (ESI) $m / z$ : found $[\mathrm{M}+\mathrm{Na}]^{+} 1153.3695$, $\mathrm{C}_{49} \mathrm{H}_{66} \mathrm{~N}_{2} \mathrm{O}_{28}$ calcd for $[\mathrm{M}+\mathrm{Na}]^{+} 1153.3694$.

[Methyl 4,7,8-tri-O-acetyl-3,5-dideoxy-9-O-methyl-5-(methyl 5-acetoxyacetamido-4,7,8-tri- $O$ - 
acetyl-3,5-dideoxy-9-O-methyl-D-glycero- $\alpha$-D-galacto-2-nonulopyranosylonat)oxyacetamido-Dglycero- $\alpha$-D-galacto-2-nonulopyranosylonat]oxyacetic acid (20)

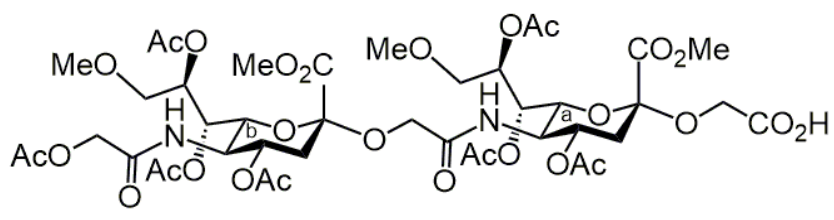

20

To a solution of $19(120 \mathrm{mg}, 106 \mu \mathrm{mol})$ in EtOAc $(3.5 \mathrm{~mL})$ was added palladium hydroxide on carbon (20\% wt. loading; $60 \mathrm{mg}$ ) at $\mathrm{rt}$. After stirring for $30 \mathrm{~min}$ at $\mathrm{rt}$ under a hydrogen atmosphere, the completion of the reaction was confirmed by TLC $\left(10: 1 \mathrm{CHCl}_{3}-\mathrm{MeOH}\right)$. The reaction mixture was filtered through a pad of Celite, washed with EtOAc. The combined filtrate and washings were concentrated to give $20(110 \mathrm{mg}$, quant. $)$ as white foamy material; $[\alpha]_{\mathrm{D}}-11.8^{\circ}\left(\mathrm{c} 0.6, \mathrm{CHCl}_{3}\right) ;{ }^{1} \mathrm{H}$ NMR (500 MHz, $\left.\mathrm{CDCl}_{3}\right) \delta$ 6.20-5.92 (2 d, $\left.2 \mathrm{H}, \mathrm{NH}^{a}, \mathrm{NH}^{b}\right), 5.35-5.24\left(\mathrm{~m}, 4 \mathrm{H}, \mathrm{H}-7^{a}, \mathrm{H}-8^{a}, \mathrm{H}-7^{b}, \mathrm{H}-\right.$ $\left.8^{b}\right), 5.04-4.95\left(\mathrm{~m}, 2 \mathrm{H}, \mathrm{H}-4^{a}, \mathrm{H}-4^{b}\right), 4.59\left(\mathrm{~d}, 1 \mathrm{H}, J_{\mathrm{gem}}=15.0 \mathrm{~Hz}, \mathrm{H}-11 \mathrm{a}^{b}\right), 4.40-4.09$ (m, 9 H, H-5, H$\left.6^{a}, \mathrm{H}-11 \mathrm{a}^{a}, \mathrm{H}-11 \mathrm{~b}^{a}, \mathrm{H}-5^{b}, \mathrm{H}-6^{b}, \mathrm{H}-11 \mathrm{~b}^{b}, \mathrm{OCH}_{2} \mathrm{CO}\right), 3.90-3.83$ (2 s, $\left.6 \mathrm{H}, 2 \mathrm{OMe}\right), 3.59-3.27$ (m, $10 \mathrm{H}$, H-9a $\left.\mathrm{a}^{a}, \mathrm{H}-9 \mathrm{~b}^{a}, \mathrm{H}-9 \mathrm{a}^{b}, \mathrm{H}-9 \mathrm{~b}^{b}, 2 \mathrm{OMe}\right), 2.75\left(\mathrm{dd}, 1 \mathrm{H}, J_{3 \mathrm{eq}, 4}=4.5 \mathrm{~Hz}, J_{\mathrm{gem}}=13.0 \mathrm{~Hz}, \mathrm{H}-3 \mathrm{eq}^{a}\right), 2.68(\mathrm{dd}$, $\left.1 \mathrm{H}, J_{\text {3eq, } 4}=4.5 \mathrm{~Hz}, J_{\text {gem }}=13.0 \mathrm{~Hz}, \mathrm{H}-3 \mathrm{eq}^{b}\right), 2.20-2.00$ (m, $\left.23 \mathrm{H}, \mathrm{H}-3 \mathrm{ax}^{a}, \mathrm{H}-3 \mathrm{ax}^{b}, 7 \mathrm{Ac}\right) ;{ }^{13} \mathrm{C} \mathrm{NMR}$ $\left(125 \mathrm{MHz}, \mathrm{CDCl}_{3}\right) \delta 171.5,171.2,171.0,170.4,170.3$ 170.1, 170.0, 170.0, 169.6, 168.8, 167.8, 167.8 , 167.7, 98.4, 98.3, 77.2, 73.1, 72.8, 71.1, 70.8, 69.1, 68.8, 68.5, 68.1, 67.6, 67.5, 63.8, 62.8, 61.8, 60.4, 59.2, 59.1, 53.3, 53.1, 49.2, 48.9, 37.6, 37.5, 21.3, 21.2, 21.0, 20.8, 20.7, 20.7, 20.7, 14.2; HRMS (ESI) $\mathrm{m} / z$ : found $[\mathrm{M}-\mathrm{H}]^{-} 1039.3259, \mathrm{C}_{42} \mathrm{H}_{59} \mathrm{~N}_{2} \mathrm{O}_{28}$ calcd for $[\mathrm{M}-\mathrm{H}]^{-} 1039.3260$.

1-D-4,5,6-Tri-O-acetyl-1-O-tert-butyldiphenylsilyl-myo-inositol (24)

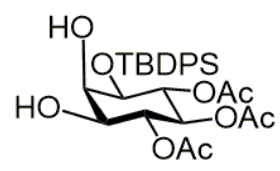

24

To a solution of 1-D-2,3-O-(D-1,7,7-trimetyl-bicyclo-[2.2.1]-hept-2-yliden)-myo-inositol ${ }^{3}$ (5.00 g, $15.9 \mathrm{mmol})$ in pyridine $(80.0 \mathrm{~mL})$ were added TBDPSCl $(6.2 \mathrm{~mL}, 23.9 \mathrm{mmol})$ and imidazole $(2.16 \mathrm{~g}$, $31.8 \mathrm{mmol}$ ) at $-10^{\circ} \mathrm{C}$. After stirring for $18 \mathrm{~h}$ at the same temperature, the completion of the reaction was confirmed by TLC $\left(8: 1 \mathrm{CHCl}_{3}-\mathrm{MeOH}\right)$. The reaction mixture was quenched with $\mathrm{MeOH}$ and then co-evaporated with toluene. The resulting residue was diluted with EtOAc and washed with water and brine. The organic layer was successively dried over $\mathrm{Na}_{2} \mathrm{SO}_{4}$, concentrated, and exposed to high vacuum for $2 \mathrm{~h}$. The residue was then dissolved in pyridine $(80.0 \mathrm{~mL})$. Acetic anhydride $(15.0 \mathrm{~mL}$, $159 \mathrm{mmol}$ ) and DMAP (389 mg, $3.18 \mathrm{mmol}$ ) were added to the mixture at $\mathrm{rt}$. After stirring for $1 \mathrm{~h}$ at $40{ }^{\circ} \mathrm{C}$, the completion of the reaction was confirmed by TLC (3:1 $n$-hexane-acetone). The reaction 
mixture was quenched with $\mathrm{MeOH}$ and then co-evaporated with toluene. The resulting residue was diluted with $\mathrm{CHCl}_{3}$ and washed with $2 \mathrm{M}$ aq. $\mathrm{HCl}, \mathrm{H}_{2} \mathrm{O}$, satd. aq. $\mathrm{NaHCO}_{3}$, and brine. The organic layer was dried over $\mathrm{Na}_{2} \mathrm{SO}_{4}$ and concentrated. The resulting residue was roughly purified by silica gel column chromatography (6:1 $n$-hexane-acetone). The purified product was then dissolved in $\mathrm{CH}_{2} \mathrm{Cl}_{2}(140 \mathrm{~mL})$ and $80 \%$ aq. TFA $(70.0 \mathrm{~mL})$ was added to the mixture at $\mathrm{rt}$. After stirring for $1 \mathrm{~h}$ at $40{ }^{\circ} \mathrm{C}$, the completion of the reaction was confirmed by TLC (2:1 $n$-hexane-acetone). The reaction mixture was co-evaporated with toluene. The resulting residue was diluted with $\mathrm{CHCl}_{3}$ and washed with satd. aq. $\mathrm{NaHCO}_{3}$ and brine. The organic layer was dried over $\mathrm{Na}_{2} \mathrm{SO}_{4}$ and concentrated. The resulting residue was purified by silica gel column chromatography ( $3: 1 n$-hexane-acetone) to give $\mathbf{2 4}$ $(6.86 \mathrm{~g}, 78 \%)$ as white foamy material; $[\alpha]_{\mathrm{D}}+38.2^{\circ}\left(\mathrm{c} 0.9, \mathrm{CHCl}_{3}\right) ;{ }^{1} \mathrm{H} \mathrm{NMR}\left(500 \mathrm{MHz}, \mathrm{CDCl}_{3}\right) \delta$ 7.68-7.37 (m, $10 \mathrm{H}, \mathrm{Ph}), 5.51\left(\mathrm{t}, 1 \mathrm{H}, J_{1,6}=J_{5,6}=10.0 \mathrm{~Hz}, \mathrm{H}-6\right), 5.34\left(\mathrm{t}, 1 \mathrm{H}, J_{3,4}=J_{4,5}=10.0 \mathrm{~Hz}, \mathrm{H}-\right.$ 4), 4.86 (t, $1 \mathrm{H}, \mathrm{H}-5$ ), 3.85 (dd, $\left.1 \mathrm{H}, J_{1,2}=2.5 \mathrm{~Hz}, J_{2,3}=2.0 \mathrm{~Hz}, \mathrm{H}-2\right), 3.77$ (dd, $\left.1 \mathrm{H}, \mathrm{H}-1\right), 3.36$ (td, 1 $\left.\mathrm{H}, J_{3, \mathrm{OH}}=10.5 \mathrm{~Hz}, \mathrm{H}-3\right), 2.76(\mathrm{~s}, 1 \mathrm{H}, 2-\mathrm{OH}), 2.62(\mathrm{~d}, 1 \mathrm{H}, 3-\mathrm{OH}), 2.02-1.66$ (m, $\left.9 \mathrm{H}, 3 \mathrm{Ac}\right), 1.06$ (s, $\left.9 \mathrm{H},{ }^{t} \mathrm{Bu}\right) ;{ }^{13} \mathrm{C} \mathrm{NMR}\left(125 \mathrm{MHz}, \mathrm{CDCl}_{3}\right) \delta 170.8,170.2,169.9,135.8,135.6,132.6,132.1,130.5,130.1$, $128.2,127.8,72.5,72.4,72.2,71.9,71.0,70.4,26.8,20.8,20.6,20.6,19.2$; HRMS (ESI) $m / z$ : found $[\mathrm{M}+\mathrm{Na}]^{+}$567.2021, $\mathrm{C}_{28} \mathrm{H}_{36} \mathrm{O} 9 \mathrm{Si}$ calcd for $[\mathrm{M}+\mathrm{Na}]^{+} 567.2021$.

1-O-[Methyl 4,7,8,9-tetra- $O$-acetyl-3,5-dideoxy-5-(2,2,2-trichloroethoxylcarbamoyl)-D-glycero-Dgalacto-2-nonulopyranosylonate]-4,5,6-tri- $O$-acetyl-1-O-tert-butyldiphenylsilyl-D-myo-inositol (26)

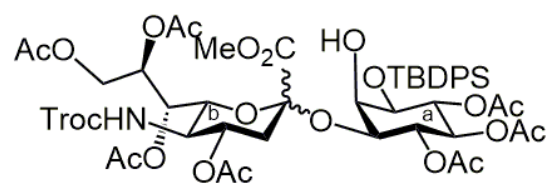

26

A solution of $21(145 \mathrm{mg}, 202 \mu \mathrm{mol})$ and $24(165 \mathrm{mg}, 303 \mu \mathrm{mol})$ in EtCN $(2.0 \mathrm{~mL})$ was transferred into a two-neck round bottom flask, in which $3 \AA$ molecular sieves $(200 \mathrm{mg})$ were placed, using a cannula, followed by addition of $N$-iodosuccinimide $(68.0 \mathrm{mg}, 302 \mu \mathrm{mol})$. The suspension was stirred for $30 \mathrm{~min}$ at $\mathrm{rt}$ and then cooled to $-50{ }^{\circ} \mathrm{C}$. Subsequently, triethylsilyl trifluoromethanesulfonate (6.9 $\mu \mathrm{L}, 10.5 \mu \mathrm{mol}$ ) was added to the mixture. After stirring for $2 \mathrm{~h}$ at the same temperature, the completion of the reaction was confirmed by TLC (3:1 toluene-EtOAc). The reaction mixture was quenched with satd. aq. $\mathrm{NaHCO}_{3}$ and then filtered through a pad of Celite, washed with $\mathrm{CHCl}_{3}$. The combined filtrate and washings were successively washed with satd. aq. $\mathrm{Na}_{2} \mathrm{~S}_{2} \mathrm{O}_{3}$ and brine, dried over $\mathrm{Na}_{2} \mathrm{SO}_{4}$, and concentrated. The resulting residue was purified by column chromatography on silica gel (3:1 $\rightarrow 1: 1$ toluene-EtOAc) and Sephadex LH-20 (1:1 CHCl $3-\mathrm{MeOH})$ to give $\mathbf{2 6 \boldsymbol { \alpha }}(134 \mathrm{mg}, 58 \%)$ as white foamy material along with $\mathbf{2 6 \beta}(17 \mathrm{mg}, 7 \%)$.

26a: $[\alpha]_{\mathrm{D}}-28.9^{\circ}\left(\mathrm{c} 1.9, \mathrm{CDCl}_{3}\right) ;{ }^{1} \mathrm{H}$ NMR $\left(500 \mathrm{MHz}, \mathrm{CDCl}_{3}\right) \delta$ 7.71-7.38 (m, $\left.10 \mathrm{H}, \mathrm{Ph}\right), 5.54-5.43$ 
$\left(\mathrm{m}, 3 \mathrm{H}, \mathrm{H}-4^{a}, \mathrm{H}-6^{a}, \mathrm{H}-8^{b}\right), 5.39\left(\mathrm{dd}, 1 \mathrm{H}, J_{6,7}=2.3 \mathrm{~Hz}, J_{7,8}=8.1 \mathrm{~Hz}, \mathrm{H}-7^{b}\right), 5.12\left(\mathrm{~d}, 1 \mathrm{H}, J_{5, \mathrm{NH}}=10.1\right.$ $\mathrm{Hz}, \mathrm{NH}), 5.07\left(\mathrm{t}, 1 \mathrm{H}, J_{3,4}=J_{4,5}=9.8 \mathrm{~Hz}, \mathrm{H}-5^{a}\right), 4.89-4.82\left(\mathrm{~m}, 2 \mathrm{H}, \mathrm{H}-4^{b}, \mathrm{CCl}_{3} \mathrm{CH} H_{2} \mathrm{O}\right), 4.44(\mathrm{~d}, 1 \mathrm{H}, J$ $\left.=12.1 \mathrm{~Hz}, \mathrm{CCl}_{3} \mathrm{CH}_{2} \mathrm{O}\right), 4.38\left(\mathrm{dd}, 1 \mathrm{H}, J_{8,9 \mathrm{a}}=2.1 \mathrm{~Hz}, J_{\mathrm{gem}}=12.1 \mathrm{~Hz}, \mathrm{H}-9 \mathrm{a}^{b}\right), 4.27\left(\mathrm{dd}, 1 \mathrm{H}, J_{1,2}=2.1\right.$ $\left.\mathrm{Hz}, J_{1,6}=10.1 \mathrm{~Hz}, \mathrm{H}-1^{a}\right), 4.03-3.99\left(\mathrm{~m}, 2 \mathrm{H}, \mathrm{H}-3^{a}, \mathrm{H}_{-} \mathrm{g}^{b}\right) 3.86\left(\mathrm{dd}, 1 \mathrm{H}, J_{5,6}=10.8 \mathrm{~Hz}, \mathrm{H}-6^{b}\right), 3.69-$ 3.65 (m, 2 H, H-2 $\left.{ }^{a}, \mathrm{H}^{-} 5^{b}\right), 3.43$ (s, $3 \mathrm{H}, \mathrm{OMe}$ ), 2.75 (brs, $\left.1 \mathrm{H}, 2-\mathrm{OH}\right), 2.70$ (dd, $1 \mathrm{H}, J_{3 \mathrm{eq}, 4}=4.5 \mathrm{~Hz}$, $\left.J_{\text {gem }}=12.5 \mathrm{~Hz}, \mathrm{H}-3 \mathrm{eq}^{b}\right), 2.14,2.05,2.00,1.99,1.96$, and $1.91(6 \mathrm{~s}, 18 \mathrm{H}, 6 \mathrm{Ac}), 2.70$ (t, $1 \mathrm{H}, J_{3 \mathrm{eq}, 4}=$ $\left.12.5 \mathrm{~Hz}, \mathrm{H}-3 \mathrm{ax}^{b}\right), 1.65$ (s, $\left.3 \mathrm{H}, \mathrm{Ac}\right), 1.04$ (s, $\left.9 \mathrm{H},{ }^{t} \mathrm{Bu}\right) ;{ }^{13} \mathrm{C} \mathrm{NMR}\left(125 \mathrm{MHz}, \mathrm{CDCl}_{3}\right) \delta 170.6,170.2$, 170.0, 169.9, 169.8, 169.4, 167.6, 154.1, 135.7, 135.5, 132.9, 132.8, 129.9, 129.8, 127.7, 12.7.7, 96.9, $95.2,74.4,72.4,72.2,71.4,71.2,70.9,70.8,69.5,68.5,68.3,67.5,62.2,52.6,51.1,37.7,26.6,21.0$, 20.7, 20.7, 20.6, 20.6, 20.5, 20.5, 19.1; Gated $\left\{{ }^{1} \mathrm{H}\right\}{ }^{13} \mathrm{C}$ NMR $\left(125 \mathrm{MHz}, \mathrm{CDCl}_{3}\right) \delta 167.8\left(J_{\mathrm{Cl}, \mathrm{H} 3 a x}=\right.$ $6.3 \mathrm{~Hz}, \mathrm{C}-1^{b}$ ); HRMS (ESI) $\mathrm{m} / z$ : found $[\mathrm{M}+\mathrm{Na}]^{+} 1172.2494, \mathrm{C}_{49} \mathrm{H}_{62} \mathrm{Cl}_{3} \mathrm{NO}_{22} \mathrm{Si}$ calcd. for $[\mathrm{M}+\mathrm{Na}]^{+} 1172.2491$.

26ß: $[\alpha]_{\mathrm{D}}+8.84{ }^{\circ}\left(\mathrm{c} 1.6, \mathrm{CDCl}_{3}\right) ;{ }^{1} \mathrm{H}$ NMR $\left(500 \mathrm{MHz}, \mathrm{CDCl}_{3}\right) \delta 7.73-7.37(\mathrm{~m}, 10 \mathrm{H}, \mathrm{Ph}), 5.54-5.43$ $\left(\mathrm{m}, 3 \mathrm{H}, \mathrm{H}-4^{a}, \mathrm{H}-6^{a}, \mathrm{H}-8^{b}\right), 5.33\left(\mathrm{~m}, 1 \mathrm{H}, \mathrm{H}-7^{b}\right), 5.13\left(\mathrm{~d}, 1 \mathrm{H}, J_{5, \mathrm{NH}}=10.1 \mathrm{~Hz}, \mathrm{NH}\right), 5.05\left(\mathrm{td}, 1 \mathrm{H}, J_{3 \mathrm{eq}, 4}\right.$ $\left.=4.8 \mathrm{~Hz}, J_{3 \mathrm{ax}, 4}=J_{4,5}=10.8 \mathrm{~Hz}, \mathrm{H}-4^{b}\right), 4,87\left(\mathrm{~d}, 1 \mathrm{H}, J_{\mathrm{gem}}=12.1 \mathrm{~Hz}, \mathrm{CCl}_{3} \mathrm{CH}_{2} \mathrm{O}\right), 4.79\left(\mathrm{t}, 1 \mathrm{H}, J_{3,4}=J_{4,5}\right.$ $\left.=9.8 \mathrm{~Hz}, \mathrm{H}-5^{a}\right), 4.55\left(\mathrm{~d}, 1 \mathrm{H}, \mathrm{CCl}_{3} \mathrm{CH}_{2} \mathrm{O}\right), 4.48\left(\mathrm{dd}, 1 \mathrm{H}, J_{8,9 \mathrm{a}}=2.5 \mathrm{~Hz}, J_{\text {gem }}=12.3 \mathrm{~Hz}, \mathrm{H}-9 \mathrm{a}^{b}\right), 4.39$ $\left(\mathrm{dd}, 1 \mathrm{H}, J_{5,6}=10.7 \mathrm{~Hz}, J_{6,7}=2.7 \mathrm{~Hz}, \mathrm{H}-6^{b}\right), 4.19\left(\mathrm{dd}, 1 \mathrm{H}, J_{8,9 \mathrm{~b}}=6.2 \mathrm{~Hz}, \mathrm{H}-9 \mathrm{~b}^{b}\right), 4.15\left(\mathrm{~m}, 1 \mathrm{H}, \mathrm{H}-2^{a}\right)$, 3.92 (q, $\left.1 \mathrm{H}, \mathrm{H}-5^{b}\right), 3.67$ (dd, $1 \mathrm{H}, J_{1,2}=2.3 \mathrm{~Hz}, J_{1,6}=9.7 \mathrm{~Hz}, \mathrm{H}-1^{a}$ ), 3.60 (s, $3 \mathrm{H}, \mathrm{OMe}$ ), 3.56 (brs, 1 H, 2-OH), $3.46\left(\mathrm{dd}, 1 \mathrm{H}, J_{2,3}=1.8 \mathrm{~Hz}, J_{3,4}=10.0 \mathrm{~Hz}, \mathrm{H}-3^{a}\right), 2.46\left(\mathrm{dd}, 1 \mathrm{H}, J_{\mathrm{gem}}=13.0 \mathrm{~Hz}, \mathrm{H}-3 \mathrm{eq}^{b}\right)$, 2.13, 2.11, 2.04, 1.99, 1.98, 1.94, and $1.80(7 \mathrm{~s}, 21 \mathrm{H}, 7 \mathrm{Ac}), 1.71\left(\mathrm{t}, 1 \mathrm{H}, \mathrm{H}-3 \mathrm{ax}^{b}\right), 1.03\left(\mathrm{~s}, 9 \mathrm{H},{ }^{t} \mathrm{Bu}\right)$; ${ }^{13} \mathrm{C}$ NMR $\left(125 \mathrm{MHz}, \mathrm{CDCl}_{3}\right) \delta 170.8,170.7,170.3,170.2,170.0,169.9,169.9,166.4,154.3,136.0$, 135.7, 133.0, 132.1, 130.2, 129.8, 127.9, 127.7, 99.0, 95.4, 74.6, 74.5, 72.2, 71.9, 71.8, 71.4, 70.9, 70.3, 69.7, 69.5, 69.0, 62.7, 52.7, 51.0, 37.4, 29.7, 26.7, 21.0, 20.9, 20.8, 20.7, 20.6, 20.4, 19.1; HRMS (ESI) $m / z$ : found $[\mathrm{M}+\mathrm{Na}]^{+} 1172.2498, \mathrm{C}_{49} \mathrm{H}_{62} \mathrm{Cl}_{3} \mathrm{NO}_{22} \mathrm{Si}$ calcd. for $[\mathrm{M}+\mathrm{Na}]^{+} 1172.2491$.

1-O-[Methyl 4,7,8-tri- $O$-acetyl-3,5-dideoxy-9-O-methyl-5-(2,2,2-trichloroethoxylcarbamoyl)-Dglycero-D-galacto-2-nonulopyranosylonate]-4,5,6-tri-O-acetyl-1-O-tert-butyldiphenylsilyl-D-myoinositol (27)

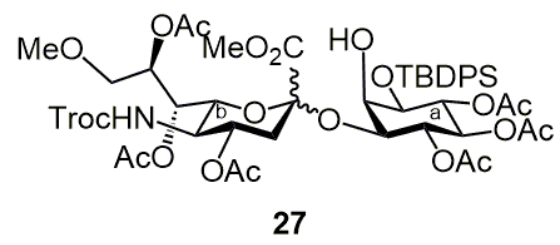

A solution of $15(500 \mathrm{mg}, 0.726 \mathrm{mmol})$ and $24(593 \mathrm{mg}, 1.09 \mathrm{mmol})$ in $\operatorname{EtCN}(7.3 \mathrm{~mL})$ was transferred into a two-neck round bottom flask, in which $3 \AA$ molecular sieves $(360 \mathrm{mg})$ were placed, using a cannula, followed by addition of $N$-iodosuccinimide ( $245 \mathrm{mg}, 1.09 \mathrm{mmol})$. The suspension was stirred 
for $30 \mathrm{~min}$ at $\mathrm{rt}$ and then cooled to $-50^{\circ} \mathrm{C}$. Subsequently, triethylsilyl trifluoromethanesulfonate (24.6 $\mu \mathrm{L}, 0.109 \mathrm{mmol}$ ) was added to the mixture. After stirring for $1.5 \mathrm{~h}$ at the same temperature, the completion of the reaction was confirmed by TLC (5:2n-hexane-EtOAc twice). The reaction mixture was quenched with satd. aq. $\mathrm{NaHCO}_{3}$ and then filtered through a pad of Celite, washed with $\mathrm{CHCl}_{3}$. The combined filtrate and washings were successively washed with satd. aq. $\mathrm{Na}_{2} \mathrm{~S}_{2} \mathrm{O}_{3}$ and brine, dried over $\mathrm{Na}_{2} \mathrm{SO}_{4}$, and concentrated. The resulting residue was purified by column chromatography on silica gel $\left(5: 2 \rightarrow 1: 1 n\right.$-hexane-EtOAc) and Sephadex LH-20 (1:1 $\left.\mathrm{CHCl}_{3}-\mathrm{MeOH}\right)$ to give $\mathbf{2 7 \alpha}(536$ $\mathrm{mg}, 66 \%$ ) as white foamy material along with $27 \boldsymbol{2}(49 \mathrm{mg}, 6 \%)$.

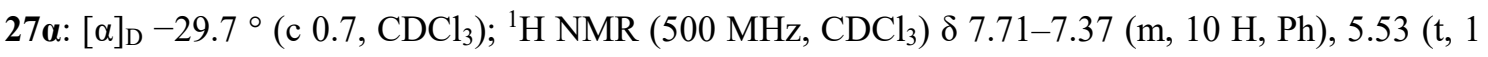
$\left.\mathrm{H}, J_{1,6}=J_{5,6}=10.0 \mathrm{~Hz}, \mathrm{H}-6^{a}\right), 5.48-5.44\left(\mathrm{~m}, 2 \mathrm{H}, \mathrm{H}-8^{b}, \mathrm{H}-4^{a}\right), 5.40\left(\mathrm{dd}, 1 \mathrm{H}, J_{6,7}=2.5 \mathrm{~Hz}, J_{7,8}=9.5\right.$ $\left.\mathrm{Hz}, \mathrm{H}-7^{b}\right), 5.12\left(\mathrm{t}, 1 \mathrm{H}, J_{4,5}=9.5 \mathrm{~Hz}, \mathrm{H}-5^{a}\right), 4.87-4.42\left(\mathrm{~m}, 3 \mathrm{H}, \mathrm{H}-4^{b}, \mathrm{CCl}_{3} \mathrm{CH}_{2} \mathrm{O}\right), 4.74\left(\mathrm{~d}, 1 \mathrm{H}, J_{5, \mathrm{NH}}\right.$ $=10.5 \mathrm{~Hz}, \mathrm{NH}), 4.20\left(\mathrm{dd}, 1 \mathrm{H}, J_{2,3}=2.5 \mathrm{~Hz}, J_{3,4}=10.0 \mathrm{~Hz}, \mathrm{H}-3^{a}\right), 4.00\left(\mathrm{dd}, 1 \mathrm{H}, J_{1,2}=2.5 \mathrm{~Hz}, \mathrm{H}-1^{a}\right)$, $3.84\left(\mathrm{dd}, 1 \mathrm{H}, J_{5,6}=10.5 \mathrm{~Hz}, \mathrm{H}-6^{b}\right), 3.66$ (q, $1 \mathrm{H}, J_{4,5}=10.5 \mathrm{~Hz}, \mathrm{H}-5^{b}$ ), 3.60 (brs, $1 \mathrm{H}, \mathrm{H}-2^{a}$ ), 3.49 (dd, $\left.1 \mathrm{H}, J_{\text {gem }}=11.5 \mathrm{~Hz}, J_{8,9 \mathrm{a}}=3.5 \mathrm{~Hz}, \mathrm{H}-9 \mathrm{a}^{b}\right), 3.42(\mathrm{~s}, 3 \mathrm{H}, \mathrm{OMe}), 3.33\left(\mathrm{dd}, 1 \mathrm{H}, J_{8,9 \mathrm{~b}}=5.0 \mathrm{~Hz}, \mathrm{H}-9 \mathrm{~b}^{b}\right)$, 3.29 (s, $3 \mathrm{H}, \mathrm{OMe}$ ), 2.69 (dd, $\left.1 \mathrm{H}, J_{\mathrm{gem}}=12.6 \mathrm{~Hz}, J_{3 \mathrm{eq}, 4}=4.5 \mathrm{~Hz}, \mathrm{H}-3 \mathrm{eq}^{b}\right), 2.49$ (s, $1 \mathrm{H}, 2-\mathrm{OH}$ ), $2.12-$ 1.65 (m, $\left.19 \mathrm{H}, \mathrm{H}-3 \mathrm{ax}^{b}, 6 \mathrm{Ac}\right), 1.03\left(\mathrm{~s}, 9 \mathrm{H},{ }^{t} \mathrm{Bu}\right) ;{ }^{13} \mathrm{C} \mathrm{NMR}\left(125 \mathrm{MHz}, \mathrm{CDCl}_{3}\right) \delta 170.3,170.0,169.9$, $169.8,169.8,169.5,167.8,154.1,135.7,135.6,133.0,132.9,130.0,129.9,127.8,127.8,96.8,95.2$, 77.2, 74.6, 72.5, 72.1, 71.4, 71.3, 70.9, 69.8, 68.6, 68.0, 67.4, 59.0, 52.6, 51.2, 37.9, 26.7, 21.2, 20.8, 20.7, 20.6, 19.3; Gated $\left\{{ }^{1} \mathrm{H}\right\}{ }^{13} \mathrm{C}$ NMR $\left(125 \mathrm{MHz}, \mathrm{CDCl}_{3}\right) \delta 167.8\left(J_{\mathrm{Cl}, \mathrm{H} 3 \mathrm{ax}}=5.0 \mathrm{~Hz}, \mathrm{C}-1^{b}\right)$; HRMS (ESI) $m / z$ : found $[\mathrm{M}+\mathrm{Na}]^{+} 1144.2541, \mathrm{C}_{48} \mathrm{H}_{62} \mathrm{Cl}_{3} \mathrm{NO}_{21} \mathrm{Si}$ calcd. for $[\mathrm{M}+\mathrm{Na}]^{+} 1144.2541$.

27ß: $[\alpha]_{\mathrm{D}}+13.8^{\circ}\left(\mathrm{c} 1.0, \mathrm{CDCl}_{3}\right) ;{ }^{1} \mathrm{H}$ NMR $\left(500 \mathrm{MHz}, \mathrm{CDCl}_{3}\right) \delta 7.75-7.35(\mathrm{~m}, 10 \mathrm{H}, \mathrm{Ph}), 5.53(\mathrm{t}, 1$ $\left.\mathrm{H}, J_{3,4}=J_{4,5}=9.5 \mathrm{~Hz}, \mathrm{H}-4^{a}\right), 5.49\left(\mathrm{t}, 1 \mathrm{H}, J_{1,6}=J_{5,6}=10.0 \mathrm{~Hz}, \mathrm{H}-6^{a}\right), 5.35\left(\mathrm{~m}, 1 \mathrm{H}, \mathrm{H}-8^{b}\right), 5.30(\mathrm{t}, 1$ $\left.\mathrm{H}, J_{6,7}=J_{7,8}=2.5 \mathrm{~Hz}, \mathrm{H}-7^{b}\right), 5.07-5.01\left(\mathrm{~m}, 2 \mathrm{H}, \mathrm{H}-4^{b}, \mathrm{NH}\right), 4.88\left(\mathrm{~d}, 1 \mathrm{H}, J_{\mathrm{gem}}=12.5 \mathrm{~Hz}, \mathrm{CCl}_{3} \mathrm{CH}_{2} \mathrm{O}\right)$, $4.76\left(\mathrm{t}, 1 \mathrm{H}, \mathrm{H}-5^{a}\right), 4.53$ (d, $\left.1 \mathrm{H}, \mathrm{CCl}_{3} \mathrm{CH}_{2} \mathrm{O}\right), 4.45\left(\mathrm{dd}, 1 \mathrm{H}, J_{5,6}=11.0 \mathrm{~Hz}, \mathrm{H}-6^{b}\right), 4.16-4.10(\mathrm{~m}, 2 \mathrm{H}$, $\left.\mathrm{H}-2^{a}, \mathrm{H}-5^{b}\right), 3.71\left(\mathrm{dd}, 1 \mathrm{H}, J_{8,9 \mathrm{a}}=2.0 \mathrm{~Hz}, J_{\mathrm{gem}}=11.0 \mathrm{~Hz}, \mathrm{H}-9 \mathrm{a}^{b}\right), 3.69(\mathrm{brs}, 1 \mathrm{H}, 2-\mathrm{OH}), 3.62-3.58(\mathrm{~m}$, $4 \mathrm{H}, \mathrm{H}-1^{a}$, OMe), 3.45 (m, $\left.1 \mathrm{H}, \mathrm{H}-9 \mathrm{~b}^{b}\right), 3.38$ (dd, $1 \mathrm{H}, J_{2,3}=2.0 \mathrm{~Hz}, \mathrm{H}-3^{a}$ ), 3.33 (s, $\left.3 \mathrm{H}, \mathrm{OMe}\right), 2.46$ $\left(\mathrm{dd}, 1 \mathrm{H}, J_{3 \mathrm{eq}, 4}=5.0 \mathrm{~Hz}, J_{\mathrm{gem}}=13.0 \mathrm{~Hz}, \mathrm{H}-3 \mathrm{eq}^{b}\right), 2.15-1.92(\mathrm{~m}, 18 \mathrm{H}, 6 \mathrm{Ac}), 1.73\left(\mathrm{t}, 1 \mathrm{H}, J_{3 \mathrm{ax}, 4}=12.0\right.$ $\left.\mathrm{Hz}, \mathrm{H}-3 \mathrm{ax}^{b}\right), 1.02\left(\mathrm{~s}, 9 \mathrm{H},{ }^{t} \mathrm{Bu}\right) ;{ }^{13} \mathrm{C}$ NMR $\left(125 \mathrm{MHz}, \mathrm{CDCl}_{3}\right) \delta 171.2,170.3,170.1,169.9,169.8$, 166.5, 154.3, 136.1, 135.7, 133.2, 132.0, 130.2, 129.7, 128.0, 127.7, 99.0, 95.4, 77.2, 75.0, 74.5, 72.3, $72.0,71.9,71.6,71.3,70.9,70.0,69.8,69.2,60.4,59.2,52.6,51.1,37.4,31.6,29.3,26.7,22.6,21.2$, 21.0, 20.9, 20.8, 20.7, 20.6, 20.4, 19.1, 14.2, 14.1; HRMS (ESI) $m / z$ : found $[\mathrm{M}+\mathrm{Na}]^{+} 1144.2540$, $\mathrm{C}_{48} \mathrm{H}_{62} \mathrm{Cl}_{3} \mathrm{NO}_{21} \mathrm{Si}$ calcd. for $[\mathrm{M}+\mathrm{Na}]^{+} 1144.2541$.

1-D-4,5,6-O-Acetyl-1-O-tert-butyldiphenylsilyl-[4',7',8'-tri- $O$-acetyl-5' -(2,2,2trichloroethoxycarbamoyl)-3',5'-dideoxy-9'-O-methyl-D-glycero- $\alpha$-D-galacto-2'nonulopyranosylono-1',2-lactone]-myo-inositol (28) 


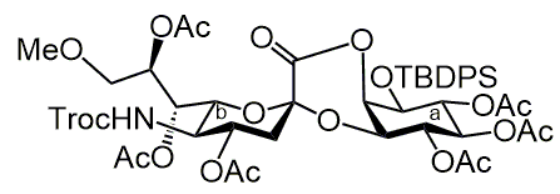

28

To a mixture of $27 \boldsymbol{\alpha}(1.24 \mathrm{~g}, 1.10 \mathrm{mmol})$ and $4 \AA$ molecular sieves $(11.0 \mathrm{~g})$ in toluene $(110 \mathrm{~mL})$ was added 1,8-diazabicyclo[5.4.0] undec-7-ene $(197 \mu \mathrm{L}, 1.32 \mathrm{mmol})$ at $\mathrm{rt}$. After stirring for $30 \mathrm{~min}$ at $100{ }^{\circ} \mathrm{C}$, the completion of the reaction was confirmed by TLC ( $2: 3 n$-hexane-EtOAc). The reaction mixture was quenched with $\mathrm{AcOH}$ and then filtered through a pad of Celite, washed with EtOAc. The combined filtrate and washings were concentrated and purified by silica gel column chromatography (3:2 $n$-hexane-EtOAc) to give 28 (1.20 g, quant.) as a white amorphous solid; $[\alpha]_{\mathrm{D}}-7.7^{\circ}$ (c 1.0 , $\mathrm{CHCl}_{3}$ ); ${ }^{1} \mathrm{H} \mathrm{NMR}\left(500 \mathrm{MHz}, \mathrm{CDCl}_{3}\right) \delta 7.70-7.30(\mathrm{~m}, 10 \mathrm{H}, \mathrm{Ph}), 5.64$ (t, $1 \mathrm{H}, J_{1,6}=J_{5,6}=9.5 \mathrm{~Hz}, \mathrm{H}-$ $\left.6^{a}\right), 5.58\left(\mathrm{~m}, 1 \mathrm{H}, \mathrm{H}-4^{b}\right), 5.31\left(\mathrm{dd}, 1 \mathrm{H}, J_{6,7}=1.0 \mathrm{~Hz}, J_{7,8}=7.0 \mathrm{~Hz}, \mathrm{H}-7^{b}\right), 5.23\left(\mathrm{t}, 1 \mathrm{H}, J_{3,4}=J_{4,5}=6.5\right.$ $\left.\mathrm{Hz}, \mathrm{H}-4^{a}\right), 5.19$ (m, $\left.1 \mathrm{H}, \mathrm{H}-8^{b}\right), 5.03-4.98$ (m, $\left.2 \mathrm{H}, \mathrm{H}-2^{a}, \mathrm{NH}\right), 4.90-4.85$ (m, $2 \mathrm{H}, \mathrm{H}-5^{a}, \mathrm{CCl}_{3} \mathrm{CH}_{2} \mathrm{O}$ ), $4.50\left(\mathrm{~d}, 1 \mathrm{H}, J_{\text {gem }}=12.0 \mathrm{~Hz}, \mathrm{CCl}_{3} \mathrm{CH}_{2} \mathrm{O}\right), 4.27\left(\mathrm{dd}, 1 \mathrm{H}, J_{1,2}=5.0 \mathrm{~Hz}, \mathrm{H}-1^{a}\right), 4.02\left(\mathrm{dd}, 1 \mathrm{H}, J_{2,3}=3.0\right.$ $\left.\mathrm{Hz}, \mathrm{H}-3^{a}\right), 3.94-3.88\left(\mathrm{~m}, 2 \mathrm{H}, \mathrm{H}-5^{b}, \mathrm{H}-6^{b}\right), 3.54\left(1 \mathrm{H}, J_{8,9 \mathrm{a}}=3.5 \mathrm{~Hz}, J_{\mathrm{gem}}=10.5 \mathrm{~Hz}, \mathrm{H}-9 \mathrm{a}^{b}\right), 3.38(\mathrm{dd}$, $\left.1 \mathrm{H}, J_{8,9 \mathrm{~b}}=6.0 \mathrm{~Hz}, \mathrm{H}-9 \mathrm{~b}^{b}\right), 3.27$ (s, $\left.3 \mathrm{H}, \mathrm{OMe}\right), 2.47\left(\mathrm{dd}, 1 \mathrm{H}, J_{3 \mathrm{eq}, 4}=5.5 \mathrm{~Hz}, J_{\text {gem }}=13.5 \mathrm{~Hz}, \mathrm{H}-3 \mathrm{eq}^{b}\right)$, 2.12-1.94 (m, $18 \mathrm{H}, 6 \mathrm{Ac}), 1.81$ (dd, $\left.1 \mathrm{H}, J_{3 \mathrm{ax}, 4}=11.0 \mathrm{~Hz}, \mathrm{H}-3 \mathrm{ax}^{b}\right), 1.05\left(\mathrm{~s}, 9 \mathrm{H},{ }^{t} \mathrm{Bu}\right) ;{ }^{13} \mathrm{C} \mathrm{NMR}(125$ $\left.\mathrm{MHz}, \mathrm{CDCl}_{3}\right) \delta 170.3,170.0,170.0,169.5,169.1,163.2,154.4,136.3,135.8,133.0,131.2$, $130.4,129.9,128.0,127.7,95.3,94.7,77.6,74.7,74.6,73.2,72.3,71.3,71.1,70.7,70.4$, $70.2,69.1,68.4,59.3,51.6,37.6,30.9,26.7,20.9,20.8,20.7,20.5,20.2,19.2$; HRMS (ESI) $m / z$ : found $[\mathrm{M}+\mathrm{Na}]^{+} 1112.2280, \mathrm{C}_{47} \mathrm{H}_{58} \mathrm{Cl}_{3} \mathrm{NO}_{20} \mathrm{Si}$ calcd for $[\mathrm{M}+\mathrm{Na}]^{+} 1112.2279$.

1-D-4,5,6-O-Acetyl-[4',7', 8'-tri-O-acetyl-5'-(2,2,2-trichloroethoxycarbamoyl)-3',5'-dideoxy-9'-Omethyl-D-glycero- $\alpha$-D-galacto-2'-nonulopyranosylono-1',2-lactone]-myo-inositol (29)

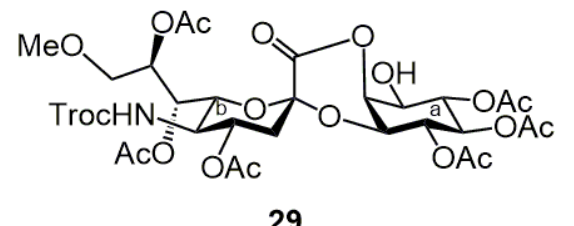

29

To a solution of $\mathbf{2 8}(323 \mathrm{mg}, 296 \mu \mathrm{mol})$ in DMF (5.9 mL) were added tris(dimethylamino)sulfonium difluorotrimethylsilicate (408 mg, $1.48 \mathrm{mmol})$ and $\mathrm{AcOH}(85.3 \mu \mathrm{L}, 1.48 \mathrm{mmol})$ at rt. After stirring for $3 \mathrm{~h}$ at $\mathrm{rt}$, the completion of the reaction was confirmed by TLC (2:3n-hexane-EtOAc). The reaction mixture was diluted with EtOAc. The organic layer was successively washed with $\mathrm{H}_{2} \mathrm{O}$ and brine, dried over $\mathrm{Na}_{2} \mathrm{SO}_{4}$, and concentrated. The resulting residue was purified by silica gel column chromatography $\left(80: 1 \mathrm{CHCl}_{3}-\mathrm{MeOH}\right)$ to give $29(249 \mathrm{mg}, 99 \%)$ as white foamy material; $[\alpha]_{\mathrm{D}}$ 
$-15.9^{\circ}\left(\mathrm{c} 1.1, \mathrm{CHCl}_{3}\right) ;{ }^{1} \mathrm{H} \mathrm{NMR}\left(500 \mathrm{MHz}, \mathrm{CDCl}_{3}\right) \delta 6.38\left(\mathrm{~d}, 1 \mathrm{H}, J_{5, \mathrm{NH}}=9.5 \mathrm{~Hz}, \mathrm{NH}\right), 5.54(\mathrm{~m}, 1$ $\left.\mathrm{H}, \mathrm{H}-4^{b}\right), 5.41-5.35\left(\mathrm{~m}, 3 \mathrm{H}, \mathrm{H}-2^{a}, \mathrm{H}-6^{a}, \mathrm{H}-7^{b}\right), 5.25-5.20\left(\mathrm{~m}, 2 \mathrm{H}, \mathrm{H}-4^{a}, \mathrm{H}-8^{b}\right), 5.12\left(\mathrm{~d}, 1 \mathrm{H}, J_{4,5}=\right.$ $\left.J_{5,6}=10.0 \mathrm{~Hz}, \mathrm{H}-5^{a}\right), 4.93\left(\mathrm{~d}, 1 \mathrm{H}, J_{\mathrm{gem}}=12.0 \mathrm{~Hz}, \mathrm{CCl}_{3} \mathrm{CH}_{2} \mathrm{O}\right), 4.45\left(\mathrm{~d}, 1 \mathrm{H}, \mathrm{CCl}_{3} \mathrm{CH}_{2} \mathrm{O}\right), 4.18(\mathrm{dd}, 1$ $\left.\mathrm{H}, J_{2,3}=3.0 \mathrm{~Hz}, J_{3,4}=10.0 \mathrm{~Hz}, \mathrm{H}-3^{a}\right), 3.98\left(\mathrm{dd}, 1 \mathrm{H}, J_{1,2}=2.5 \mathrm{~Hz}, J_{1,6}=9.5 \mathrm{~Hz}, \mathrm{H}-1^{a}\right), 3.85-3.76(\mathrm{~m}$, $\left.2 \mathrm{H}, \mathrm{H}-5^{b}, \mathrm{H}-6^{b}\right), 3.48\left(\mathrm{dd}, 1 \mathrm{H}, J_{8,9 \mathrm{a}}=3.5 \mathrm{~Hz}, J_{\mathrm{gem}}=10.5 \mathrm{~Hz}, \mathrm{H}-9 \mathrm{a}^{b}\right), 3.38\left(\mathrm{dd}, 1 \mathrm{H}, J_{8,9 \mathrm{~b}}=6.0 \mathrm{~Hz}, \mathrm{H}-\right.$ $\left.9 \mathrm{~b}^{b}\right), 3.29(\mathrm{~s}, 3 \mathrm{H}, \mathrm{OMe}), 2.50\left(\mathrm{dd}, J_{3 \mathrm{eq}, 4}=5.5 \mathrm{~Hz}, J_{\mathrm{gem}}=13.5 \mathrm{~Hz}, \mathrm{H}-3 \mathrm{eq}^{b}\right), 2.28-1.96(\mathrm{~m}, 18 \mathrm{H}, 6 \mathrm{Ac})$, $1.74\left(\mathrm{dd}, 1 \mathrm{H}, J_{3 \mathrm{ax}, 4}=11.5 \mathrm{~Hz}, \mathrm{H}-3 \mathrm{ax}^{b}\right) ;{ }^{13} \mathrm{C} \mathrm{NMR}\left(125 \mathrm{MHz}, \mathrm{CDCl}_{3}\right) \delta 172.4,170.4,170.0,169.9$, $169.9,169.6,162.9,162.6,154.5,95.7,95.3,77.6,74.6,74.5,72.2,71.3,71.3,71.0,70.0$, 69.4, 69.2, 68.9, 68.0, 67.1, 59.3, 51.7, 37.7, 36.5, 31.5, 30.9, 21.0, 20.9, 20.8, 20.7, 20.6, 20.5; HRMS (ESI) $m / z$ : found $[\mathrm{M}+\mathrm{Na}]^{+} 874.1103, \mathrm{C}_{31} \mathrm{H}_{40} \mathrm{Cl}_{3} \mathrm{NO}_{20}$ calcd for $[\mathrm{M}+\mathrm{Na}]^{+} 874.1101$.

1-D-4,5,6-O-Acetyl-[4',7', 8'-tri- $O$-acetyl-5'-acetoxyacetamido-3',5'-dideoxy-9'-O-methyl-Dglycero- $\alpha$-D-galacto-2'-nonulopyranosylono-1',2-lactone]-myo-inositol (30)

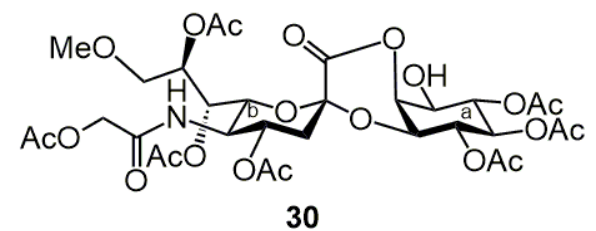

To a solution of $29(200 \mathrm{mg}, 234 \mu \mathrm{mol})$ in $\mathrm{MeCN}-\mathrm{AcOH}(4: 1,2.3 \mathrm{~mL})$ was added $\mathrm{Zn}$ powder $(1.00$ $\mathrm{g}$, excess) at rt. After stirring for $30 \mathrm{~min}$ at $\mathrm{rt}$, the completion of the reaction was confirmed by TLC (15:1 $\left.\mathrm{CHCl}_{3}-\mathrm{MeOH}\right)$. The reaction mixture was filtered through a pad of Celite, washed with $\mathrm{CHCl}_{3}$. The combined filtrate and washings were successively washed with satd. aq. $\mathrm{NaHCO}_{3}$ and brine, dried over $\mathrm{Na}_{2} \mathrm{SO}_{4}$, concentrated, and exposed to high vacuum for $1 \mathrm{~h}$. The residue was then dissolved in THF $(2.3 \mathrm{~mL})$. Acetoxyacetyl chloride $(25.2 \mu \mathrm{L}, 234 \mu \mathrm{mol})$ and triethylamine $(32.6 \mu \mathrm{L}, 234 \mu \mathrm{mol})$ were added to the mixture at $0{ }^{\circ} \mathrm{C}$. After stirring for $10 \mathrm{~min}$ at the same temperature, the completion of the reaction was confirmed by TLC (1:3 toluene-EtOAc). The reaction mixture was diluted with $\mathrm{CHCl}_{3}$. The organic layer was successively washed with $\mathrm{H}_{2} \mathrm{O}$, dried over $\mathrm{Na}_{2} \mathrm{SO}_{4}$, and concentrated. The resulting residue was purified by silica gel column chromatography $\left(80: 1 \mathrm{CHCl}_{3}-\mathrm{MeOH}\right)$ to give $30(163 \mathrm{mg}, 90 \%)$ as white foamy material; $[\alpha]_{\mathrm{D}}-3.9^{\circ}\left(\mathrm{c} 1.0, \mathrm{CDCl}_{3}\right) ;{ }^{1} \mathrm{H} \mathrm{NMR}\left(500 \mathrm{MHz}, \mathrm{CDCl}_{3}\right)$ $\delta 5.91\left(\mathrm{~d}, 1 \mathrm{H}, J_{5, \mathrm{NH}}=10.5 \mathrm{~Hz}, \mathrm{NH}\right), 5.54\left(\mathrm{td}, 1 \mathrm{H}, J_{3 \mathrm{eq}, 4}=5.5 \mathrm{~Hz}, J_{3 \mathrm{ax}, 4}=J_{4,5}=11.5 \mathrm{~Hz}, \mathrm{H}-4^{b}\right), 5.40$ (t, $\left.1 \mathrm{H}, J_{1,6}=J_{5,6}=10.0 \mathrm{~Hz}, \mathrm{H}-6^{a}\right), 5.29\left(\mathrm{t}, 1 \mathrm{H}, J_{1,2}=J_{2,3}=2.0 \mathrm{~Hz}, \mathrm{H}-2^{a}\right), 5.25-5.17\left(\mathrm{~m}, 3 \mathrm{H}, \mathrm{H}-4^{a}\right.$, $\left.\mathrm{H}-7^{b}, \mathrm{H}-8^{b}\right), 5.12\left(\mathrm{t}, 1 \mathrm{H}, J_{4,5}=10.0 \mathrm{~Hz}, \mathrm{H}-5^{a}\right), 4.65\left(\mathrm{~d}, 1 \mathrm{H}, J_{\mathrm{gem}}=15.5 \mathrm{~Hz}, \mathrm{H}-11 \mathrm{a}^{b}\right), 4.27(\mathrm{~d}, 1 \mathrm{H}, \mathrm{H}-$ $\left.11 \mathrm{~b}^{b}\right), 4.20\left(\mathrm{q}, 1 \mathrm{H}, J_{5,6}=10.5 \mathrm{~Hz}, \mathrm{H}-5^{b}\right), 4.12\left(\mathrm{dd}, 1 \mathrm{H}, J_{3,4}=10.0 \mathrm{~Hz}, \mathrm{H}-3^{a}\right), 3.95\left(\mathrm{td}, 1 \mathrm{H}, J_{1, \mathrm{OH}}=2.5\right.$ Hz, 1-OH), $3.68\left(\mathrm{dd}, 1 \mathrm{H}, \mathrm{H}-6^{b}\right), 3.45\left(\mathrm{dd}, 1 \mathrm{H}, J_{8,9 \mathrm{a}}=3.5 \mathrm{~Hz}, J_{\mathrm{gem}}=11.0 \mathrm{~Hz}, \mathrm{H}-9 \mathrm{a}^{b}\right), 3.32(\mathrm{dd}, 1 \mathrm{H}$, $\left.J_{8,9 \mathrm{~b}}=5.5 \mathrm{~Hz}, \mathrm{H}-9 \mathrm{~b}^{b}\right), 3.28(\mathrm{~s}, 3 \mathrm{H}, \mathrm{OMe}), 2.48\left(\mathrm{dd}, 1 \mathrm{H}, J_{\mathrm{gem}}=14.0 \mathrm{~Hz}, \mathrm{H}-3 \mathrm{eq}^{b}\right), 2.23-2.00(\mathrm{~m}, 21$ $\mathrm{H}, 7 \mathrm{Ac}), 1.83$ (dd, $\left.1 \mathrm{H}, \mathrm{H}-3 \mathrm{ax}^{b}\right) ;{ }^{13} \mathrm{C}$ NMR $\left(125 \mathrm{MHz}, \mathrm{CDCl}_{3}\right) \delta 171.1,170.5,170.3,170.1,169.8$, 169.7, 169.6, 167.8, 163.1, 128.2, 95.5, 95.4, 77.6, 73.3, 72.8, 71.7, 71.2, 70.9, 70.7, 70.4, 70.3, 69.5, 
69.1, 68.9, 68.8, 68.6, 68.5, 67.1, 62.7, 59.1, 49.4, 38.2, 31.9, 29.7, 29.4, 22.7, 21.0, 20.8, 20.8, 20.7, 20.7, 20.6, 20.6, 14.1; HRMS (ESI) $\mathrm{m} / z$ : found $[\mathrm{M}+\mathrm{Na}]^{+} 800.2220, \mathrm{C}_{32} \mathrm{H}_{43} \mathrm{NO}_{21}$ calcd. for $[\mathrm{M}+\mathrm{Na}]^{+} 800.2220$.

1-D-4,5,6-O-Acetyl-[4',7',8'-tri- $O$-acetyl-3',5'-dideoxy-9'-O-methyl-5-(methyl 5acetoxyacetamido-4,7,8-tri-O-acetyl-3,5-dideoxy-9-O-methyl-D-glycero- $\alpha$-D-galacto-2nonulopyranosylonat)oxyacetamido-D-glycero- $\alpha$-D-galacto-2'-nonulopyranosylono-1',2-lactone]myo-inositol (31)

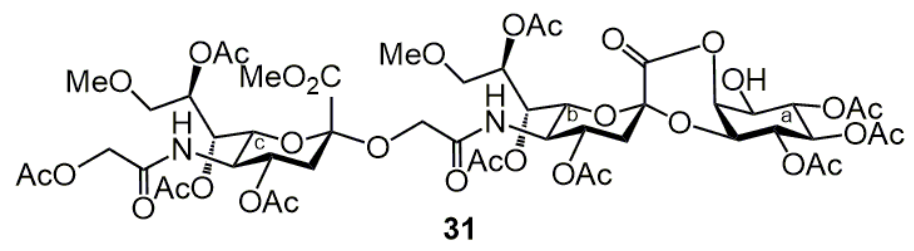

To a solution of $29(50 \mathrm{mg}, 58.6 \mu \mathrm{mol})$ in $\mathrm{MeCN}-\mathrm{AcOH}(4: 1,1.2 \mathrm{~mL})$ was added $\mathrm{Zn}$ powder $(250$ $\mathrm{mg}$, excess) at rt. After stirring for $20 \mathrm{~min}$ at $\mathrm{rt}$, the completion of the reaction was confirmed by TLC $\left(15: 1 \mathrm{CHCl}_{3}-\mathrm{MeOH}\right)$. The reaction mixture was filtered through a pad of Celite, washed with $\mathrm{CHCl}_{3}$. The combined filtrate and washings were successively washed with satd. aq. $\mathrm{NaHCO}_{3}$ and brine, dried over $\mathrm{Na}_{2} \mathrm{SO}_{4}$, concentrated, and exposed to high vacuum for $1 \mathrm{~h}$. The residue was then dissolved in DMF $(1.2 \mathrm{~mL}) .18(34 \mathrm{mg}, 58.6 \mu \mathrm{mol})$ and HBTU $(27 \mathrm{mg}, 70.3 \mu \mathrm{mol})$ were added to the mixture at rt. The mixture was stirred for $2 \mathrm{~h}$ at the same temperature, followed by addition of DIEA ( $16 \mu \mathrm{L}, 87.9$ $\mu \mathrm{mol})$. After stirring for additional $30 \mathrm{~min}$, the completion of the reaction was confirmed by TLC (15:1 $\mathrm{CHCl}_{3}-\mathrm{MeOH}$ ). The reaction mixture was diluted with EtOAc. The organic layer was successively washed with $\mathrm{H}_{2} \mathrm{O}$ and brine, dried over $\mathrm{Na}_{2} \mathrm{SO}_{4}$, and concentrated. The resulting residue was purified by silica gel column chromatography $\left(60: 1 \mathrm{CHCl}_{3}-\mathrm{MeOH}\right)$ to give $31(53 \mathrm{mg}, 73 \%)$ as a white amorphous solid; $[\alpha]_{\mathrm{D}}-31.8^{\circ}\left(\mathrm{c} 0.3, \mathrm{CDCl}_{3}\right) ;{ }^{1} \mathrm{H}$ NMR $\left(500 \mathrm{MHz}, \mathrm{CDCl}_{3}\right) \delta 6.25\left(\mathrm{~d}, 1 \mathrm{H}, J_{5, \mathrm{NH}}=10.5\right.$ $\left.\mathrm{Hz}, \mathrm{NH}^{b}\right), 5.91\left(\mathrm{~d}, 1 \mathrm{H}, J_{5, \mathrm{NH}}=10.5 \mathrm{~Hz}, \mathrm{NH}^{c}\right), 5.56\left(\mathrm{td}, 1 \mathrm{H}, J_{3 \mathrm{eq}, 4}=5.0 \mathrm{~Hz}, J_{3 \mathrm{ax}, 4}=J_{4,5}=9.5 \mathrm{~Hz}, \mathrm{H}-\right.$ $\left.4^{b}\right), 5.39\left(\mathrm{t}, 1 \mathrm{H}, J_{1,6}=J_{5,6}=9.5 \mathrm{~Hz}, \mathrm{H}-6^{a}\right), 5.33-5.19\left(\mathrm{~m}, 6 \mathrm{H}, \mathrm{H}-2^{a}, \mathrm{H}-4^{a}, \mathrm{H}-7^{b}, \mathrm{H}-8^{b}, \mathrm{H}-7^{c}, \mathrm{H}-8^{c}\right)$, $5.11\left(\mathrm{t}, 1 \mathrm{H}, J_{4,5}=10.0 \mathrm{~Hz}, \mathrm{H}-5^{a}\right), 5.00\left(\mathrm{td}, 1 \mathrm{H}, J_{3 \mathrm{eq}, 4}=5.5 \mathrm{~Hz}, J_{3 \mathrm{ax}, 4}=J_{4,5}=10.5 \mathrm{~Hz}, \mathrm{H}-4^{c}\right), 4.58(\mathrm{~d}$, $\left.1 \mathrm{H}, J_{\text {gem }}=15.0 \mathrm{~Hz}, \mathrm{H}-11 \mathrm{a}^{c}\right), 4.33\left(\mathrm{~d}, 1 \mathrm{H}, \mathrm{H}-11 \mathrm{~b}^{c}\right), 4.26-4.08\left(\mathrm{~m}, 5 \mathrm{H}, \mathrm{H}-3^{a}, \mathrm{H}-5^{b}, \mathrm{H}-5^{c}, \mathrm{H}-6^{c}, \mathrm{H}-\right.$ $\left.11 \mathrm{a}^{b}\right), 3.96\left(\mathrm{~d}, 1 \mathrm{H}, \mathrm{H}-1^{a}\right), 3.88(\mathrm{~s}, 3 \mathrm{H}, \mathrm{OMe}), 3.85\left(\mathrm{~d}, 1 \mathrm{H}, J_{1, \mathrm{OH}}=2.0 \mathrm{~Hz}, 1-\mathrm{OH}\right), 3.80\left(\mathrm{~d}, 1 \mathrm{H}, J_{\text {gem }}\right.$ $\left.=15.0 \mathrm{~Hz}, \mathrm{H}-11 \mathrm{~b}^{b}\right), 3.72\left(\mathrm{~d}, 1 \mathrm{H}, J_{5,6}=10.5 \mathrm{~Hz}, \mathrm{H}-6^{\mathrm{b}}\right), 3.58-3.25$ (m, $10 \mathrm{H}, \mathrm{H}-9 \mathrm{a}^{b}, \mathrm{H}-9 \mathrm{~b}^{b}, \mathrm{H}-9 \mathrm{a}^{c}, \mathrm{H}-$ $\left.9 \mathrm{~b}^{c}, 2 \mathrm{OMe}\right), 2.70\left(\mathrm{dd}, 1 \mathrm{H}, J_{\mathrm{gem}}=12.5 \mathrm{~Hz}, \mathrm{H}-3 \mathrm{eq}^{c}\right), 2.51\left(\mathrm{dd}, 1 \mathrm{H}, J_{\mathrm{gem}}=13.5 \mathrm{~Hz}, \mathrm{H}-3 \mathrm{eq}^{b}\right), 2.20$ $2.00\left(\mathrm{~m}, 31 \mathrm{H}, \mathrm{H}-3 \mathrm{ax}^{c}, 10 \mathrm{Ac}\right), 1.80$ (dd, $\left.1 \mathrm{H}, \mathrm{H}-3 \mathrm{ax}^{b}\right) ;{ }^{13} \mathrm{C} \mathrm{NMR}\left(125 \mathrm{MHz}, \mathrm{CDCl}_{3}\right) \delta$ 172.6, 171.0, 170.2, 170.1, 169.9, 169.9, 169.8, 169.6, 169.6, 168.8, 167.8, 167.6, 162.9, 98.4, 95.8, 77.2, 74.5, 73.0, $72.3,71.3,71.2,71.0,70.8,70.0,69.4,69.1,69.1,68.6,68.0,68.0,67.7,66.8,63.7,62.8,59.2,59.2$, 53.2, 49.3, 48.8, 37.8, 37.6, 29.7, 21.2, 21.1, 20.8, 20.7, 20.7, 20.5, 20.5; HRMS (ESI) $\mathrm{m} / z$ : found 
$[\mathrm{M}+\mathrm{Na}]^{+} 1261.3755, \mathrm{C}_{32} \mathrm{H}_{43} \mathrm{NO}_{21}$ calcd. for $[\mathrm{M}+\mathrm{Na}]^{+} 1261.3753$.

1-D-4,5,6-O-Acetyl- \{4', 7', ,' 'tri- $O$-acetyl-3',5'-dideoxy-9'-O-methyl-5'-[methyl 4,7,8-tri- $O$-acetyl3,5-dideoxy-9-O-methyl-5-(methyl 5-acetoxyacetamido-4,7,8-tri- $O$-acetyl-3,5-dideoxy-9-O-methylD-glycero- $\alpha$-D-galacto-2-nonulopyranosylonat)oxyacetamido-D-glycero- $\alpha$-D-galacto-2nonulopyranosylonat]oxyacetamido-D-glycero- $\alpha$-D-galacto-2'-nonulopyranosylono-1',2-lactone\}myo-inositol (32)

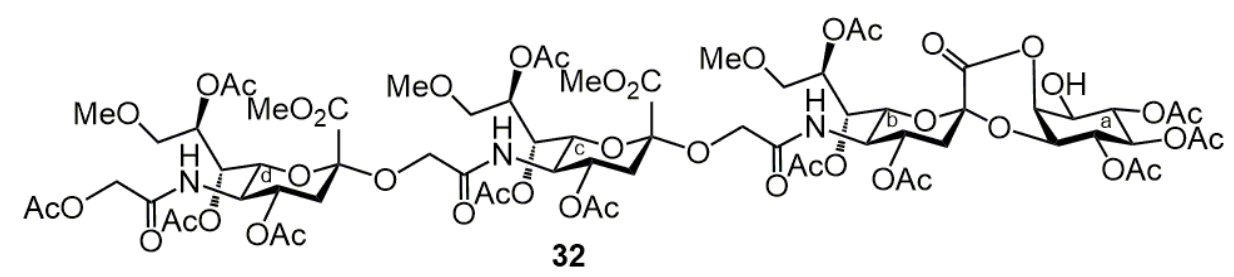

To a solution of 29 (46 mg, $53.9 \mu \mathrm{mol})$ in $\mathrm{MeCN}-\mathrm{AcOH}(4: 1,1.9 \mathrm{~mL})$ was added $\mathrm{Zn}$ powder (230 $\mathrm{mg}$, excess) at rt. After stirring for $30 \mathrm{~min}$ at $\mathrm{rt}$, the completion of the reaction was confirmed by TLC $\left(20: 1 \mathrm{CHCl}_{3}-\mathrm{MeOH}\right)$. The reaction mixture was filtered through a pad of Celite, washed with $\mathrm{CHCl}_{3}$. The combined filtrate and washings were successively washed with satd. aq. $\mathrm{NaHCO}_{3}$ and brine, dried over $\mathrm{Na}_{2} \mathrm{SO}_{4}$, concentrated, and exposed to high vacuum for $1 \mathrm{~h}$. The residue was then dissolved in DMF (1.8 mL). 20 (56 mg, $53.8 \mu \mathrm{mol})$ and HBTU $(25 \mathrm{mg}, 64.6 \mu \mathrm{mol})$ were added to the mixture at rt. The mixture was stirred for $2 \mathrm{~h}$ at the same temperature, followed by addition of DIEA ( $15 \mu \mathrm{L}, 80.7$ $\mu \mathrm{mol})$. After stirring for additional $30 \mathrm{~min}$, the completion of the reaction was confirmed by TLC (15:1 $\mathrm{CHCl}_{3}-\mathrm{MeOH}$ ). The reaction mixture was diluted with EtOAc. The organic layer was successively washed with $\mathrm{H}_{2} \mathrm{O}$ and brine, dried over $\mathrm{Na}_{2} \mathrm{SO}_{4}$, and concentrated. The resulting residue was purified by silica gel column chromatography $\left(50: 1 \rightarrow 30: 1 \mathrm{CHCl}_{3}-\mathrm{MeOH}\right)$ to give $\mathbf{3 2}(68 \mathrm{mg}, 74 \%)$ as a white amorphous solid; $[\alpha]_{\mathrm{D}}-54.3^{\circ}\left(\mathrm{c} 1.0, \mathrm{CHCl}_{3}\right) ;{ }^{1} \mathrm{H} \mathrm{NMR}\left(500 \mathrm{MHz}, \mathrm{CDCl}_{3}\right) \delta 6.25-5.84(3 \mathrm{~d}, 3$ $\left.\mathrm{H}, \mathrm{NH}^{b}, \mathrm{NH}^{c}, \mathrm{NH}^{d}\right), 5.56\left(\mathrm{td}, 1 \mathrm{H}, J_{3 \mathrm{eq}, 4}=5.0 \mathrm{~Hz}, J_{3 \mathrm{ax}, 4}=J_{4,5}=11.0 \mathrm{~Hz}, \mathrm{H}-4^{b}\right), 5.39\left(\mathrm{t}, 1 \mathrm{H}, J_{1,6}=J_{5,6}\right.$ $\left.=10.0 \mathrm{~Hz}, \mathrm{H}-6^{a}\right), 5.34-5.19\left(\mathrm{~m}, 8 \mathrm{H}, \mathrm{H}-2^{a}, \mathrm{H}^{-} 4^{a}, \mathrm{H}-7^{b}, \mathrm{H}-8^{b}, \mathrm{H}-7^{c}, \mathrm{H}-8^{c}, \mathrm{H}-7^{d}, \mathrm{H}-8^{d}\right), 5.11\left(\mathrm{t}, 1 \mathrm{H}, J_{4,5}\right.$ $\left.=10.0 \mathrm{~Hz}, \mathrm{H}-5^{a}\right), 5.01-4.95\left(\mathrm{~m}, 2 \mathrm{H}, \mathrm{H}-4^{c}, \mathrm{H}-4^{d}\right), 4.59\left(\mathrm{~d}, 1 \mathrm{H}, J_{\text {gem }}=15.5 \mathrm{~Hz}, \mathrm{H}-11 \mathrm{a}^{d}\right), 4.32(\mathrm{~d}, 1 \mathrm{H}$, H-11b $b^{d}$ ), 4.25-4.10 (m, 8 H, H-3 $\left.{ }^{a}, \mathrm{H}-5^{b}, \mathrm{H}-11 \mathrm{a}^{b}, \mathrm{H}-5^{c}, \mathrm{H}^{c} 6^{c}, \mathrm{H}-11 \mathrm{a}^{c}, \mathrm{H}-5^{d}, \mathrm{H}-6^{d}\right), 3.95$ (d, $1 \mathrm{H}, \mathrm{H}-1^{a}$ ), 3.90-3.78 (m, 8 H, H-11b $\left.\mathrm{b}^{b}, \mathrm{H}-11 \mathrm{~b}^{c}, 2 \mathrm{OMe}\right), 3.71\left(\mathrm{~d}, 1 \mathrm{H}, J_{5,6}=10.5 \mathrm{~Hz}, \mathrm{H}-6^{b}\right), 3.60-3.26(\mathrm{~m}, 15 \mathrm{H}$, H-9a $\mathrm{a}^{b}, \mathrm{H}-9 \mathrm{~b}^{b}, \mathrm{H}-9 \mathrm{a}^{c}, \mathrm{H}-9 \mathrm{~b}^{c}, \mathrm{H}-9 \mathrm{a}^{d}, \mathrm{H}-9 \mathrm{~b}^{d}, 3$ OMe), 2.76-2.67 (m, 2 H, H-3eq ${ }^{c}, \mathrm{H}-3 \mathrm{eq}^{d}$ ), 2.52 (dd, 1 $\left.\mathrm{H}, J_{\text {gem }}=13.5 \mathrm{~Hz}, \mathrm{H}-3 \mathrm{eq}^{b}\right), 2.20-2.00\left(\mathrm{~m}, 41 \mathrm{H}, \mathrm{H}-3 \mathrm{ax}^{c}, \mathrm{H}-3 \mathrm{ax}^{d}, 13 \mathrm{Ac}\right), 1.81\left(\mathrm{dd}, 1 \mathrm{H}, \mathrm{H}-3 \mathrm{ax}^{b}\right) ;{ }^{13} \mathrm{C}$ NMR $\left(125 \mathrm{MHz}, \mathrm{CDCl}_{3}\right) \delta 172.7,171.0,170.5,170.2,170.1,170.0,170.0,169.9,169.9,169.6,169.6$, 168.9, 168.6, 167.8, 167.6, 162.9, 98.4, 98.4, 95.8, 77.3, 74.5, 73.1, 73.1, 72.3, 71.3, 71.0, 70.9, 70.7, $70.0,69.4,69.1,68.9,68.6,68.4,68.1,67.9,67.6,66.7,63.9,63.7,62.8,59.2,59.2,59.1,53.3,53.2$, 49.2, 48.8, 48.7, 37.8, 37.7, 37.6, 29.7, 23.0, 21.2, 21.2, 21.1, 20.9, 20.8, 20.8, 20.7, 20.7, 20.6, 20.5; HRMS (ESI) $m / z$ : found $[\mathrm{M}+\mathrm{Na}]^{+} 1722.5288, \mathrm{C}_{70} \mathrm{H}_{97} \mathrm{~N}_{3} \mathrm{O}_{45}$ calcd for $[\mathrm{M}+\mathrm{Na}]^{+} 1722.5286$. 
$(2 S, 3 R, 4 E)$-2-Tetracosanamido-hexadec-4-ene-1,3-diol (S1)

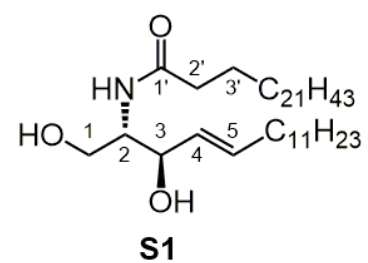

To a solution of $(2 S, 3 R, 4 E)$-2-tert-butoxycarbamoyl-hexadec-4-ene-1,3-diol ${ }^{4}(3.97 \mathrm{~g}, 10.7 \mathrm{mmol})$ in $\mathrm{CH}_{2} \mathrm{Cl}_{2}(70 \mathrm{~mL})$ were added anisole $(1.28 \mathrm{~mL}, 10.7 \mathrm{mmol})$ and $80 \%$ aq. TFA $(35 \mathrm{~mL})$ at rt. After stirring at rt for $1.5 \mathrm{~h}$, the completion of the reaction was confirmed by TLC $\left(5: 1 \mathrm{CHCl}_{3}-\mathrm{MeOH}\right)$. The reaction mixture was co-evaporated with EtOH. The resulting residue was dissolved in $\mathrm{MeOH}-\mathrm{H}_{2} \mathrm{O}$ $(1: 1,350 \mathrm{~mL}) . \mathrm{K}_{2} \mathrm{CO}_{3}(14.8 \mathrm{~g}, 107 \mathrm{mmol})$ was added to the mixture at $\mathrm{rt}$. After stirring at $\mathrm{rt}$ for $2 \mathrm{~h}$, the completion of the reaction was confirmed by TLC $\left(5: 1 \mathrm{CHCl}_{3}-\mathrm{MeOH}\right)$. The reaction mixture was neutralized with Muromac $\left(\mathrm{H}^{+}\right)$resin and then filtered through cotton wool, washing with $\mathrm{MeOH}$. The combined filtrate and washings were evaporated and dissolved in $\mathrm{CHCl}_{3}$. The resulting solution was successively washed with $\mathrm{H}_{2} \mathrm{O}$, dried over $\mathrm{Na}_{2} \mathrm{SO}_{4}$, concentrated, and exposed to high vacuum for 3 h. The residue was dissolved in $\mathrm{CHCl}_{3}(214 \mathrm{~mL})$ and tetracosanoic acid $N$-hydroxysuccinimide ester ${ }^{5}$ $(5.96 \mathrm{~g}, 12.8 \mathrm{mmol})$ and triethylamine $(150 \mu \mathrm{L}, 1.07 \mathrm{mmol})$ were added to the mixture at $\mathrm{rt}$. After stirring at $\mathrm{rt}$ for $2 \mathrm{~h}$, the completion of the reaction was confirmed by TLC $\left(10: 1 \mathrm{CHCl}_{3}-\mathrm{MeOH}\right)$. The reaction mixture was diluted with $\mathrm{CHCl}_{3}$. The resulting solution was successively washed with $\mathrm{H}_{2} \mathrm{O}$, dried over $\mathrm{Na}_{2} \mathrm{SO}_{4}$, and concentrated. The resulting residue was purified by silica gel column chromatography $\left(60: 1 \mathrm{CHCl}_{3}-\mathrm{MeOH}\right)$ to give $\mathbf{S 1}(5.51 \mathrm{~g}, 83 \%)$ as colorless syrup; $[\alpha]_{\mathrm{D}}+4.0^{\circ}(\mathrm{c} 0.8$, $\mathrm{CHCl}_{3}$ ); ${ }^{1} \mathrm{H} \mathrm{NMR}\left(500 \mathrm{MHz}, \mathrm{CDCl}_{3}\right) \delta 6.24\left(\mathrm{~d}, 1 \mathrm{H}, J_{2, \mathrm{NH}}=7.5 \mathrm{~Hz}, \mathrm{NH}\right), 5.79(\mathrm{~m}, 1 \mathrm{H}, \mathrm{H}-5), 5.53$ (dd, $\left.1 \mathrm{H}, J_{3,4}=6.5 \mathrm{~Hz}, J_{4,5}=15.5 \mathrm{~Hz}, \mathrm{H}-4\right), 4.32$ (dd, $\left.1 \mathrm{H}, J_{3, \mathrm{OH}}=5.0 \mathrm{~Hz}, \mathrm{H}-3\right), 3.98-3.90$ (m, $2 \mathrm{H}, \mathrm{H}-2, \mathrm{H}-$ 1a), 3.73-3.69 (m, $1 \mathrm{H}, \mathrm{H}-1 \mathrm{~b}), 2.71-2.68$ (m, 2 H, 1-OH, 3-OH), 2.23 (t, $2 \mathrm{H}, J_{2,3}=7.4 \mathrm{~Hz}, \mathrm{H}-2$ '), 2.06 (m, 2 H, H-6), 1.65-1.57 (m, 2 H, H-3'), 1.38-1.21 (m, 58 H, $29 \mathrm{CH}_{2}$ ), 0.88 (m, $6 \mathrm{H}, 2 \mathrm{Me}$ ); ${ }^{13} \mathrm{C}$ NMR $\left(125 \mathrm{MHz}, \mathrm{CDCl}_{3}\right) \delta 173.9,134.3,128.8,77.2,74.7,62.5,54.5,36.9,32.3,31.9,29.7,29.7$, 29.7, 29.6, 29.6, 29.5, 29.5, 29.3, 29.3, 29.2, 29.1, 25.8, 22.7, 14.1; HRMS (ESI) $m / z$ : found $[\mathrm{M}+\mathrm{Na}]^{+} 644.5952, \mathrm{C}_{40} \mathrm{H}_{79} \mathrm{NO}_{3}$ calcd for $[\mathrm{M}+\mathrm{Na}]^{+} 644.5952$.

$(2 S, 3 R, 4 E)$-2-Tetracosanamido-1-O-trityl-hexadec-4-ene-1,3-diol (S2)

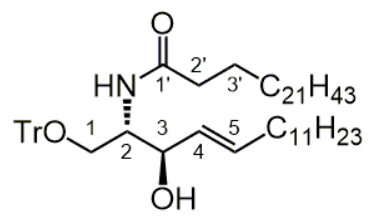

s2 
To a solution of $\mathbf{S} 1(500 \mathrm{mg}, 800 \mu \mathrm{mol})$ in pyridine $(8.0 \mathrm{~mL})$ were added $\operatorname{TrCl}(558 \mathrm{mg}, 2.01 \mathrm{mmol})$ and DMAP $(10 \mathrm{mg}, 80 \mu \mathrm{mol})$ at $\mathrm{rt}$. After stirring at $50^{\circ} \mathrm{C}$ for $11 \mathrm{~h}$, the completion of the reaction was confirmed by TLC (30:1 $\left.\mathrm{CHCl}_{3}-\mathrm{MeOH}\right)$. The reaction mixture was quenched with $\mathrm{MeOH}$ and then co-evaporated with toluene. The resulting residue was diluted with $\mathrm{CHCl}_{3}$ and washed with $2 \mathrm{M}$ aq. $\mathrm{HCl}, \mathrm{H}_{2} \mathrm{O}$, satd. aq. $\mathrm{NaHCO}_{3}$, and brine. The organic layer was dried over $\mathrm{Na}_{2} \mathrm{SO}_{4}$ and concentrated. The resulting residue was purified by silica gel column chromatography $\left(20: 1 \mathrm{CHCl}_{3}-\mathrm{MeOH}\right)$ to give S2 $(587 \mathrm{mg}, 84 \%)$ as colorless syrup; $[\alpha]_{\mathrm{D}}+0.5^{\circ}\left(\mathrm{c} 1.0, \mathrm{CHCl}_{3}\right) ;{ }^{1} \mathrm{H} \mathrm{NMR}\left(500 \mathrm{MHz}, \mathrm{CDCl}_{3}\right) \delta 7.42-$ $7.23(\mathrm{~m}, 15 \mathrm{H}, \mathrm{Ph}), 6.06$ (d, $\left.1 \mathrm{H}, J_{2, \mathrm{NH}}=8.0 \mathrm{~Hz}, \mathrm{NH}\right), 5.63\left(\mathrm{~m}, 1 \mathrm{H}, \mathrm{H}-5^{a}\right), 5.26\left(\mathrm{dd}, 1 \mathrm{H}, J_{3,4}=6.5 \mathrm{~Hz}\right.$, $\left.J_{4,5}=14.5 \mathrm{~Hz}, \mathrm{H}-4^{a}\right), 4.17\left(\mathrm{~m}, 1 \mathrm{H}, \mathrm{H}-3^{a}\right), 4.06\left(\mathrm{~m}, 1 \mathrm{H}, \mathrm{H}-2^{a}\right), 3.40-3.37\left(\mathrm{~m}, 2 \mathrm{H}, \mathrm{H}-1 \mathrm{a}^{a}, 3-\mathrm{OH}\right), 3.30$ $\left(\mathrm{dd}, 1 \mathrm{H}, J_{1 \mathrm{~b}, 2}=4.0 \mathrm{~Hz}, J_{\mathrm{gem}}=10.0 \mathrm{~Hz}, \mathrm{H}-1 \mathrm{~b}^{a}\right), 2.20\left(\mathrm{~m}, 2 \mathrm{H}, \mathrm{H}-2^{b}\right), 1.93\left(\mathrm{~m}, 2 \mathrm{H}, \mathrm{H}-6^{a}\right), 1.64(\mathrm{~m}, 2 \mathrm{H}$, $\mathrm{H}-3^{b}$ ), 1.31-1.13 (m, $58 \mathrm{H}, 29 \mathrm{CH}_{2}$ ), 0.89-0.87 (m, $6 \mathrm{H}, 2 \mathrm{Me}$ ); ${ }^{13} \mathrm{C} \mathrm{NMR}\left(125 \mathrm{MHz}, \mathrm{CDCl}_{3}\right) \delta 173.9$, 143.3, 133.5, 128.7, 128.5, 128.0, 127.3, 87.4, 77.6, 74.4, 74.3, 63.1, 53.4, 36.9, 32.2, 31.0, 29.72, $29.67,29.65,29.53,29.45,29.39,29.37,29.3,29.1,26.9,22.7,14.1$; HRMS (ESI) $m / z$ : found $[\mathrm{M}+\mathrm{Na}]^{+} 886.7048, \mathrm{C}_{59} \mathrm{H}_{93} \mathrm{NO}_{3}$ calcd for $[\mathrm{M}+\mathrm{Na}]^{+} 886.7048$.

(2S,3R,4E)-3-O-Benzoyl-2-tetracosanamido-hexadec-4-ene-1,3-diol (34)

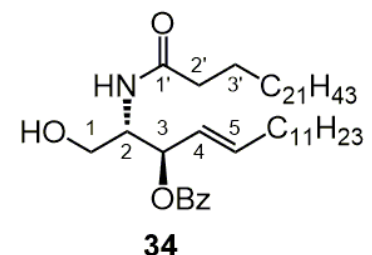

To a solution of $\mathbf{S 2}(2.71 \mathrm{~g}, 3.14 \mathrm{mmol})$ in pyridine $(31.4 \mathrm{~mL})$ were added benzoic anhydride $(1.41 \mathrm{~g}$, $6.27 \mathrm{mmol}$ ) and DMAP (38 mg, $314 \mu \mathrm{mol}$ ) at rt. After stirring at $40{ }^{\circ} \mathrm{C}$ for $12 \mathrm{~h}$, the completion of the reaction was confirmed by TLC (5:1 $n$-hexane-EtOAc). The reaction mixture was quenched with $\mathrm{MeOH}$ and then co-evaporated with toluene. The resulting residue was diluted with $\mathrm{CHCl}_{3}$ and washed with $2 \mathrm{M}$ aq. $\mathrm{HCl}, \mathrm{H}_{2} \mathrm{O}$, satd. aq. $\mathrm{NaHCO}_{3}$, and brine. The organic layer was successively dried over $\mathrm{Na}_{2} \mathrm{SO}_{4}$, concentrated, and exposed to high vacuum for $2 \mathrm{~h}$. The residue was then dissolved in $\mathrm{CHCl}_{3}-$ $\mathrm{MeOH}(20: 1,63 \mathrm{~mL})$. TsOH $\cdot \mathrm{H}_{2} \mathrm{O}(240 \mathrm{mg}, 1.26 \mathrm{mmol})$ was added to the mixture at rt. After stirring at $40{ }^{\circ} \mathrm{C}$ for $12 \mathrm{~h}$, the completion of the reaction was confirmed by TLC (3:1 $n$-hexane-EtOAc). The reaction mixture was diluted with $\mathrm{CHCl}_{3}$ and washed with satd. aq. $\mathrm{NaHCO}_{3}$ and brine. The organic layer was dried over $\mathrm{Na}_{2} \mathrm{SO}_{4}$ and concentrated. The resulting residue was purified by silica gel column chromatography (3:1 $n$-hexane-EtOAc) to give $34(2.00 \mathrm{~g}, 87 \%)$ as a white amorphous solid; $[\alpha]_{\mathrm{D}}$ $+18.3^{\circ}\left(\mathrm{c} 2.7, \mathrm{CHCl}_{3}\right) ;{ }^{1} \mathrm{H}$ NMR $\left(500 \mathrm{MHz}, \mathrm{CDCl}_{3}\right) \delta 8.04-7.44(\mathrm{~m}, 5 \mathrm{H}, \mathrm{Ph}), 6.08\left(\mathrm{~d}, 1 \mathrm{H}, J_{2, \mathrm{NH}}=\right.$ $8.5 \mathrm{~Hz}, \mathrm{NH}), 5.89-5.83\left(\mathrm{~m}, 1 \mathrm{H}, \mathrm{H}-5^{a}\right), 5.86\left(\mathrm{dd}, 1 \mathrm{H}, J_{3,4}=7.5 \mathrm{~Hz}, J_{4,5}=15.0 \mathrm{~Hz}, \mathrm{H}-4^{a}\right), 5.54(\mathrm{t}, 1 \mathrm{H}$, $\left.J_{2,3}=7.5 \mathrm{~Hz}, \mathrm{H}-3^{a}\right), 4.29-4.26\left(\mathrm{~m}, 1 \mathrm{H}, \mathrm{H}-2^{a}\right), 3.75-3.71$ (m, $\left.2 \mathrm{H}, \mathrm{H}-1 \mathrm{a}^{a}, \mathrm{H}-1 \mathrm{~b}^{a}\right), 2.97$ (brs, $1 \mathrm{H}, 1-$ $\mathrm{OH}), 2.22-2.17\left(\mathrm{~m}, 1 \mathrm{H}, \mathrm{H}-2^{b}\right) 2.05\left(\mathrm{~m}, 2 \mathrm{H}, \mathrm{H}-6^{a}\right), 1.64-1.58\left(\mathrm{~m}, 2 \mathrm{H}, \mathrm{H}-3^{b}\right), 1.29-1.11$ (m, $58 \mathrm{H}, 29$ 
$\left.\mathrm{CH}_{2}\right), 0.92-0.84$ (m, $\left.6 \mathrm{H}, 2 \mathrm{Me}\right) ;{ }^{13} \mathrm{C} \mathrm{NMR}\left(125 \mathrm{MHz}, \mathrm{CDCl}_{3}\right) \delta 173.4,166.5,137.5,133.4,129.8$, 129.7, 128.5, 124.8, 74.7, 61.9, 53.5, 36.9, 32.3, 31.9, 29.7, 29.7, 29.6, 29.6, 29.5, 29.5, 29.4, 29.3, 29.3, 29.2, 28.9, 25.7, 22.7, 14.1; HRMS (ESI) $m / z$ : found $[\mathrm{M}+\mathrm{Na}]^{+} 748.6214, \mathrm{C}_{47} \mathrm{H}_{83} \mathrm{NO}_{4}$ calcd for $[\mathrm{M}+\mathrm{Na}]^{+} 748.6214$.

1-D-4,5,6-O-Acetyl-(4',7', 8'-tri-O-acetyl-5'-acetoxyacetamido-3',5'-dideoxy-9'-O-methyl-Dglycero- $\alpha$-D-galacto-2'-nonulopyranosylono-1',2-lactone)-1-O-\{[(2S,3R,4E)-3-O-benzoyl-2-Otetracosamido-hexadec-4-ene-1,3-diol-1-yl](2-cyanoethoxy)phosphoryl\}-myo-inositol (36)

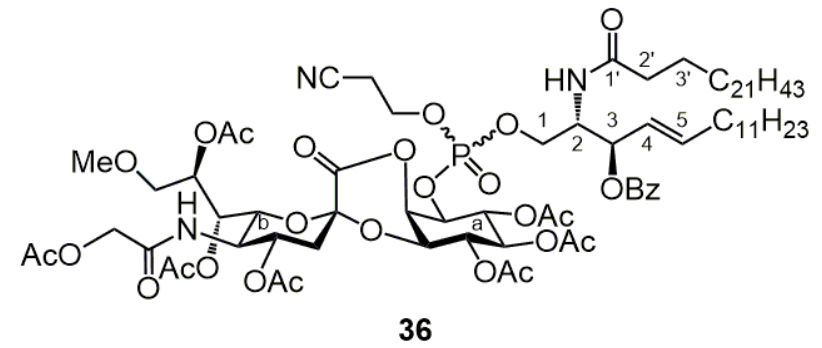

For preparation of 6: To a solution of $34(140 \mathrm{mg}, 193 \mu \mathrm{mol})$ and DIEA ( $68 \mu \mathrm{L}, 386 \mu \mathrm{mol})$ in $\mathrm{CH}_{2} \mathrm{Cl}_{2}$ $(1.93 \mathrm{~mL})$ was added 2-cyanoethyl $N, N$-diisopropylchlorophosphoramidite $35(65 \mu \mathrm{L}, 290 \mu \mathrm{mol})$ at rt. After stirring for $10 \mathrm{~min}$, the mixture was concentrated to a quarter of the original volume and passed through short silica gel column chromatography (15:1 $n$-hexane-EtOAc containing 3\% triethylamine) to give $\mathbf{6}$, which was immediately used for the following coupling reaction due to its instability.

To a solution of $30(50 \mathrm{mg}, 64.3 \mu \mathrm{mol})$ in $\mathrm{CH}_{2} \mathrm{Cl}_{2}-\mathrm{MeCN}(1: 1,1.1 \mathrm{~mL})$ were added $1 H$-tetrazole $(27 \mathrm{mg}, 386 \mu \mathrm{mol})$ and $4 \AA$ molecular sieves $(210 \mathrm{mg})$ at $\mathrm{rt}$ and the mixture was stirred for $30 \mathrm{~min}$ at $\mathrm{rt}$. After addition of a solution of the crude 6 in $\mathrm{CH}_{2} \mathrm{Cl}_{2}-\mathrm{MeCN}(1: 1,1.1 \mathrm{~mL})$ and stirring for additional $10 \mathrm{~min}$ at $\mathrm{rt}$, the completion of the reaction was confirmed by TLC ( $3: 1 \mathrm{CHCl}_{3}$-acetone). The reaction mixture then was treated with $5.5 \mathrm{M}$ solution of TBHP in decane $(58 \mu \mathrm{L}, 322$ $\mu \mathrm{mol})$. After stirring for extra $10 \mathrm{~min}$, the completion of the reaction was confirmed by TLC (3:1 $\mathrm{CHCl}_{3}$-acetone). The reaction mixture was diluted with $\mathrm{CHCl}_{3}$, washed with brine. The organic layer was dried over $\mathrm{Na}_{2} \mathrm{SO}_{4}$, and concentrated. The resulting residue was purified by silica gel column chromatography (6:1 $\mathrm{CHCl}_{3}$-acetone) to give $\mathbf{3 6}$ (72 $\mathrm{mg}, 69 \%$, diastereomeric mixture) as a white amorphous solid. The diastereomers were partially separated by silica gel column chromatography (6:1 $\mathrm{CHCl}_{3}$-acetone) to give $\mathbf{3 6} \mathbf{a}$ and $\mathbf{3 6} \mathbf{b}$ in pure forms.

36a: $[\alpha]_{\mathrm{D}}-13.3^{\circ}\left(\mathrm{c} 0.8, \mathrm{CHCl}_{3}\right) ;{ }^{1} \mathrm{H}$ NMR $\left(500 \mathrm{MHz}, \mathrm{CD}_{3} \mathrm{CN}\right) \delta$ 7.98-7.40 (m, $\left.5 \mathrm{H}, \mathrm{Ph}\right), 6.85(\mathrm{~d}, 1$ $\left.\mathrm{H}, J_{2, \mathrm{NH}}=9.5 \mathrm{~Hz}, \mathrm{NHCO}^{C e r}\right), 5.97\left(\mathrm{~d}, 1 \mathrm{H}, J_{5, \mathrm{NH}}=10.0 \mathrm{~Hz}, \mathrm{NH}^{b}\right), 5.81\left(\mathrm{~m}, 1 \mathrm{H}, \mathrm{H}-5^{C e r}\right), 5.49-5.37$ (m, $4 \mathrm{H}, \mathrm{H}-2^{a}, \mathrm{H}-4^{b}, \mathrm{H}-3^{C e r}$, H-4 ${ }^{\mathrm{Cer}}$ ), 5.31 (t, $1 \mathrm{H}, J_{1,6}=J_{5,6}=9.5 \mathrm{~Hz}, \mathrm{H}-6^{a}$ ), 5.17-5.00 (m, $4 \mathrm{H}, \mathrm{H}-4^{a}$, $\mathrm{H}-5^{a}, \mathrm{H}-7^{b}, \mathrm{H}-8^{b}$ ), 4.67 (td, $1 \mathrm{H}, J_{1,2}=3.0 \mathrm{~Hz}, J_{1, \mathrm{P}}=10.0 \mathrm{~Hz}, \mathrm{H}-1^{a}$ ), $4.45\left(\mathrm{~m}, 1 \mathrm{H}, \mathrm{H}-2^{\mathrm{Cer}}\right.$ ), 4.36 (dd, 1 $\left.\mathrm{H}, J_{2,3}=3.5 \mathrm{~Hz}, J_{3,4}=9.5 \mathrm{~Hz}, \mathrm{H}-3^{a}\right), 4.32\left(\mathrm{~d}, 1 \mathrm{H}, J_{\mathrm{gem}}=15.0 \mathrm{~Hz}, \mathrm{H}-11 \mathrm{a}^{b}\right), 4.15\left(\mathrm{~d}, 1 \mathrm{H}, \mathrm{H}-11 \mathrm{~b}^{b}\right)$, 
4.14-4.07 (m, $4 \mathrm{H}, \mathrm{H}-1 \mathrm{a}^{\mathrm{Cer}}, \mathrm{H}-1 \mathrm{~b}^{\mathrm{Cer}}, \mathrm{OCH}_{2} \mathrm{CH}_{2} \mathrm{CN}$ ), 4.00 (q, $1 \mathrm{H}, J_{4,5}=J_{5,6}=J_{5, \mathrm{NH}}=9.5 \mathrm{~Hz}, \mathrm{H}-5^{b}$ ), $3.90\left(\mathrm{dd}, 1 \mathrm{H}, J_{6,7}=1.0 \mathrm{~Hz}, \mathrm{H}-6^{b}\right), 3.38\left(\mathrm{dd}, 1 \mathrm{H}, J_{8,9 \mathrm{a}}=3.0 \mathrm{~Hz}, J_{\mathrm{gem}}=10.5 \mathrm{~Hz}, \mathrm{H}-9 \mathrm{a}^{b}\right), 3.29(\mathrm{dd}, 1 \mathrm{H}$, $\left.J_{8,9 \mathrm{~b}}=4.0 \mathrm{~Hz}, \mathrm{H}-9 \mathrm{~b}^{b}\right), 3.14(\mathrm{~s}, 3 \mathrm{H}, \mathrm{OMe}), 2.67\left(\mathrm{~m}, 2 \mathrm{H}, \mathrm{OCH}_{2} \mathrm{CH}_{2} \mathrm{CN}\right), 2.35\left(\mathrm{dd}, 1 \mathrm{H}, J_{3 \mathrm{eq}, 4}=5.5 \mathrm{~Hz}\right.$, $\left.J_{\text {gem }}=13.5 \mathrm{~Hz}, \mathrm{H}-3 \mathrm{eq}^{b}\right), 2.20-1.85\left(\mathrm{~m}, 25 \mathrm{H}, \mathrm{H}-6^{\mathrm{Cer}}, \mathrm{H}-2^{\text {, }}{ }^{\mathrm{Cer}}, 7 \mathrm{Ac}\right), 1.60\left(\mathrm{dd}, 1 \mathrm{H}, J_{3 \mathrm{ax}, 4}=11.5 \mathrm{~Hz}, \mathrm{H}-\right.$ $\left.3 \mathrm{ax}^{b}\right), 1.47$ (m, $2 \mathrm{H}, \mathrm{H}-3^{\text {Cer }}$ ), 1.32-1.00 (m, $\left.58 \mathrm{H}, 29 \mathrm{CH}_{2}\right), 0.82-0.77$ (m, $\left.6 \mathrm{H}, 2 \mathrm{Me}\right) ;{ }^{13} \mathrm{C}$ NMR $(125$ $\left.\mathrm{MHz}, \mathrm{CDCl}_{3}\right) \delta 173.0,171.1,171.0,170.1,170.1,169.6,169.6,169.2,167.8,166.0,162.6,138.1$, 133.6, 129.8, 129.7, 129.6, 128.7, 128.3, 124.7, 116.4, 95.5, 77.6, 74.3, 72.9, 72.2, 70.5, 69.8, 69.6, $69.3,68.6,68.5,67.7,66.9,62.9,62.8,62.7,59.2,50.5,50.5,50.0,38.5,36.5,32.3,31.9,29.7,29.7$, 29.7, 29.6, 29.6, 29.5, 29.5, 29.4, 29.4, 29.3, 29.2, 29.1, 28.8, 28.0, 26.1, 25.7, 22.7, 22.7, 20.9, 20.9, 20.8, 20.6, 20.4, 19.6, 19.6, 14.1; ${ }^{31} \mathrm{P}$ NMR $\left(202 \mathrm{MHz}, \mathrm{CDCl}_{3}\right) \delta-0.81$; HRMS (ESI) $m / z$ : found $[\mathrm{M}+\mathrm{Na}]^{+} 1640.8363, \mathrm{C}_{82} \mathrm{H}_{128} \mathrm{~N}_{3} \mathrm{O}_{27} \mathrm{P}$ calcd for $[\mathrm{M}+\mathrm{Na}]^{+} 1640.8365$.

36b: $[\alpha]_{\mathrm{D}}-25.8^{\circ}\left(\mathrm{c} 0.8, \mathrm{CHCl}_{3}\right) ;{ }^{1} \mathrm{H}$ NMR $\left(500 \mathrm{MHz}, \mathrm{CDCl}_{3}\right) \delta 8.06-7.45(\mathrm{~m}, 5 \mathrm{H}, \mathrm{Ph}), 6.44(\mathrm{~d}, 1 \mathrm{H}$, $\left.J_{2, \mathrm{NH}}=9.5 \mathrm{~Hz}, \mathrm{NHCO}\right), 5.97\left(\mathrm{~d}, 1 \mathrm{H}, J_{5, \mathrm{NH}}=10.5 \mathrm{~Hz}, \mathrm{NH}^{b}\right), 5.91\left(\mathrm{~m}, 1 \mathrm{H}, \mathrm{H}-5^{c}\right), 5.61-5.55(\mathrm{~m}, 2 \mathrm{H}$, $\left.\mathrm{H}-4^{b}, \mathrm{H}-3^{c}\right), 5.57-5.44\left(\mathrm{~m}, 2 \mathrm{H}, \mathrm{H}-6^{a}, \mathrm{H}-4^{c}\right), 5.33\left(\mathrm{t}, 1 \mathrm{H}, J_{1,2}=J_{2,3}=3.0 \mathrm{~Hz}, \mathrm{H}-2^{a}\right), 5.30\left(\mathrm{t}, 1 \mathrm{H}, J_{3,4}\right.$ $\left.=J_{4,5}=8.0 \mathrm{~Hz}, \mathrm{H}-4^{a}\right), 5.24\left(\mathrm{dd}, 1 \mathrm{H}, J_{6,7}=2.0 \mathrm{~Hz}, J_{7,8}=8.0 \mathrm{~Hz}, \mathrm{H}-7^{b}\right), 5.19\left(\mathrm{~m}, 1 \mathrm{H}, \mathrm{H}-8^{b}\right), 5.12(\mathrm{t}, 1$ $\left.\mathrm{H}, J_{5,6}=8.0 \mathrm{~Hz}\right), 4.78\left(\mathrm{~m}, 1 \mathrm{H}, \mathrm{H}-1^{a}\right), 4.66-4.60\left(\mathrm{~m}, 2 \mathrm{H}, \mathrm{H}-2^{c}, \mathrm{H}-11 \mathrm{a}^{b}\right), 4.49\left(\mathrm{dd}, 1 \mathrm{H}, \mathrm{H}-3^{a}\right), 4.33-$ $4.08\left(\mathrm{~m}, 6 \mathrm{H}, \mathrm{H}-5^{b}, \mathrm{H}-11 \mathrm{~b}^{b}, \mathrm{H}-1 \mathrm{a}^{c}, \mathrm{H}-1 \mathrm{~b}^{c}, \mathrm{OCH}_{2} \mathrm{CH}_{2} \mathrm{CN}\right.$ ), 3.88 (dd, $1 \mathrm{H}, J_{5,6}=2.0 \mathrm{~Hz}, \mathrm{H}-6^{b}$ ), 3.55 (dd, $\left.1 \mathrm{H}, J_{8,9 \mathrm{a}}=3.0 \mathrm{~Hz}, J_{\mathrm{gem}}=10.5 \mathrm{~Hz}, \mathrm{H}-9 \mathrm{a}^{b}\right), 3.37\left(\mathrm{dd}, 1 \mathrm{H}, J_{8,9 \mathrm{~b}}=5.5 \mathrm{~Hz}, \mathrm{H}-9 \mathrm{~b}^{b}\right), 3.30(\mathrm{~s}, 3 \mathrm{H}, \mathrm{OMe})$, 2.70-2.54 (m, $2 \mathrm{H}, \mathrm{OCH}_{2} \mathrm{CH}_{2} \mathrm{CN}$ ), $2.49\left(\mathrm{dd}, 1 \mathrm{H}, J_{3 \mathrm{eq}, 4}=5.0 \mathrm{~Hz}, J_{\mathrm{gem}}=13.5 \mathrm{~Hz}, \mathrm{H}-3 \mathrm{eq}^{b}\right), 2.28-1.96$ $\left(\mathrm{m}, 25 \mathrm{H}, \mathrm{H}-6^{c}, \mathrm{H}-2^{d}, 7 \mathrm{Ac}\right), 1.84\left(\mathrm{dd}, 1 \mathrm{H}, J_{3 \mathrm{ax}, 4}=11.5 \mathrm{~Hz}, \mathrm{H}-3 \mathrm{ax}^{b}\right), 1.65-1.58\left(\mathrm{~m}, 2 \mathrm{H}, \mathrm{H}-3^{d}\right), 1.40-$ $1.10\left(\mathrm{~m}, 58 \mathrm{H}, 29 \mathrm{CH}_{2}\right), 0.91-0.85(\mathrm{~m}, 6 \mathrm{H}, 2 \mathrm{Me}) ;{ }^{13} \mathrm{C} \mathrm{NMR}\left(125 \mathrm{MHz}, \mathrm{CDCl}_{3}\right) \delta 173.2,171.1,170.3$, 170.2, 169.6, 169.4, 169.3, 167.8, 165.4, 163.1, 138.4, 133.4, 129.8, 129.7, 128.6, 124.4, 116.7, 95.4, 77.6, 73.4, 73.0, 72.7, 71.0, 69.8, 69.6, 69.4, 68.5, 68.4, 67.7, 67.3, 62.8, 62.8, 62.7, 59.2, 49.2, 37.9, $36.6,32.3,31.9,29.8,29.7,29.7,29.6,29.6,29.6,29.5,29.4,29.4,29.3,28.9,25.7,22.7,20.9,20.9$, 20.8, 20.8, 20.6, 20.5, 19.4, 19.3, 14.1; ${ }^{31} \mathrm{P}$ NMR $\left(202 \mathrm{MHz}, \mathrm{CDCl}_{3}\right) \delta-2.70$; HRMS (ESI) $m / z$ : found $[\mathrm{M}+\mathrm{Na}]^{+} 1640.8365, \mathrm{C}_{82} \mathrm{H}_{128} \mathrm{~N}_{3} \mathrm{O}_{27} \mathrm{P}$ calcd for $[\mathrm{M}+\mathrm{Na}]^{+} 1640.8365$.

1-D-4,5,6-O-Acetyl-[4',7',8'-tri-O-acetyl-5' -acetoxyacetamido-3',5'-dideoxy-9'-O-methyl-5(methyl 5-acetoxyacetamido-4,7,8-tri- $O$-acetyl-3,5-dideoxy-9-O-methyl-D-glycero- $\alpha$-D-galacto-2nonulopyranosylonat)oxyacetamido-D-glycero- $\alpha$-D-galacto-2'-nonulopyranosylono-1',2-lactone]-1$O$ - $\{[(2 S, 3 R, 4 E)-3-O$-benzoyl-2-O-tetracosamido-hexadec-4-ene-1,3-diol-1-yl](2cyanoethoxy)phosphoryl $\}$-myo-inositol (37) 


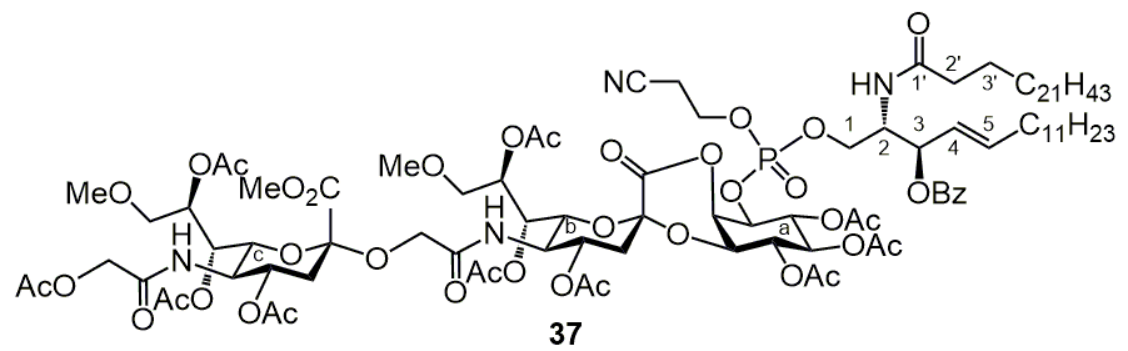

For preparation of 6: To a solution of $\mathbf{3 4}(57 \mathrm{mg}, 79.0 \mu \mathrm{mol})$ and DIEA ( $28 \mu \mathrm{L}, 158 \mu \mathrm{mol})$ in $\mathrm{CH}_{2} \mathrm{Cl}_{2}$ (790 $\mu \mathrm{L}$ ) was added 2-cyanoethyl $N, N$-diisopropylchlorophosphoramidite $35(27 \mu \mathrm{L}, 119 \mu \mathrm{mol})$ at rt. After stirring for $10 \mathrm{~min}$, the mixture was concentrated to a quarter of the original volume and passed through short silica gel column chromatography (15:1 $n$-hexane-EtOAc containing 3\% triethylamine) to give $\mathbf{6}$, which was immediately used for the following coupling reaction due to its instability.

To a solution of $31(49 \mathrm{mg}, 39.5 \mu \mathrm{mol})$ in $\mathrm{CH}_{2} \mathrm{Cl}_{2}-\mathrm{MeCN}(1: 1,2.0 \mathrm{~mL})$ were added $1 H$-tetrazole $(11 \mathrm{mg}, 158 \mu \mathrm{mol})$ and $4 \AA$ molecular sieves $(400 \mathrm{mg})$ at $\mathrm{rt}$ and the mixture was stirred for $30 \mathrm{~min}$ at $\mathrm{rt}$. After addition of a solution of the crude $\mathbf{6}$ in $\mathrm{CH}_{2} \mathrm{Cl}_{2}-\mathrm{MeCN}(1: 1,2.0 \mathrm{~mL})$ and stirring for additional $10 \mathrm{~min}$ at $\mathrm{rt}$, the completion of the reaction was confirmed by TLC (2:1 $\mathrm{CHCl}_{3}$-acetone). The reaction mixture then was treated with $5.5 \mathrm{M}$ solution of TBHP in decane $(36 \mu \mathrm{L}, 198$ $\mu \mathrm{mol})$. After stirring for extra $10 \mathrm{~min}$, the completion of the reaction was confirmed by TLC (2:1 $\mathrm{CHCl}_{3}$-acetone). The reaction mixture was diluted with $\mathrm{CHCl}_{3}$, washed with brine. The organic layer was dried over $\mathrm{Na}_{2} \mathrm{SO}_{4}$, and concentrated. The resulting residue was purified by silica gel column chromatography (3:1 $\mathrm{CHCl}_{3}$-acetone) to give $37(55 \mathrm{mg}, 67 \%$, single diastereomer) as a white amorphous solid; $[\alpha]_{\mathrm{D}}-11.6^{\circ}$ (c $\left.0.7, \mathrm{CHCl}_{3}\right) ;{ }^{1} \mathrm{H}$ NMR $\left(500 \mathrm{MHz}, \mathrm{CDCl}_{3}\right) \delta 8.07-7.44(\mathrm{~m}, 5 \mathrm{H}, \mathrm{Ph})$, $6.40\left(\mathrm{~d}, 1 \mathrm{H}, J_{2, \mathrm{NH}}=9.5 \mathrm{~Hz}, \mathrm{NHCO}^{\mathrm{Cer}}\right), 6.32\left(\mathrm{~d}, 1 \mathrm{H}, J_{5, \mathrm{NH}}=10.5 \mathrm{~Hz}, \mathrm{NH}^{b}\right), 5.93\left(\mathrm{~m}, 1 \mathrm{H}, \mathrm{H}-5^{C e r}\right)$, $5.86\left(\mathrm{~d}, 1 \mathrm{H}, J_{5, \mathrm{NH}}=10.0 \mathrm{~Hz}, \mathrm{NH}^{c}\right), 5.61-5.45\left(\mathrm{~m}, 4 \mathrm{H}, \mathrm{H}-6^{a}, \mathrm{H}-4^{b}, \mathrm{H}-3^{\mathrm{Cer}}, \mathrm{H}-4^{C e r}\right), 5.37\left(\mathrm{t}, 1 \mathrm{H}, J_{1,2}=\right.$ $\left.J_{2,3}=2.0 \mathrm{~Hz}, \mathrm{H}-2^{a}\right), 5.33-5.17\left(\mathrm{~m}, 5 \mathrm{H}, \mathrm{H}-4^{a}, \mathrm{H}-7^{b}, \mathrm{H}-8^{b}, \mathrm{H}-7^{c}, \mathrm{H}-8^{c}\right), 5.14\left(\mathrm{t}, 1 \mathrm{H}, J_{4,5}=J_{5,6}=8.0 \mathrm{~Hz}\right.$, $\left.\mathrm{H}-5^{a}\right), 5.00\left(\mathrm{~m}, 1 \mathrm{H}, \mathrm{H}-4^{c}\right), 4.78\left(\mathrm{~m}, 1 \mathrm{H}, \mathrm{H}-1^{a}\right), 4.64-4.57$ (m, $\left.2 \mathrm{H}, \mathrm{H}-11 \mathrm{a}^{c}, \mathrm{H}-2^{\mathrm{Cer}}\right), 4.34-4.08$ (m, 7 H, H-5 $\left.{ }^{b}, \mathrm{H}-11 \mathrm{a}^{b}, \mathrm{H}-5^{c}, \mathrm{H}-6^{c}, \mathrm{H}-11 \mathrm{~b}^{c}, \mathrm{OCH}_{2} \mathrm{CH}_{2} \mathrm{CN}\right), 3.91-3.88\left(\mathrm{~m}, 3 \mathrm{H}, \mathrm{H}-1 \mathrm{a}^{\mathrm{Cer}}, \mathrm{H}-1 \mathrm{~b}^{\mathrm{Cer}}, \mathrm{H}-6^{b}\right), 3.80$ $\left(\mathrm{d}, 1 \mathrm{H}, J_{\text {gem }}=15.0 \mathrm{~Hz}, \mathrm{H}-11 \mathrm{~b}^{b}\right), 3.58-3.34\left(\mathrm{~m}, 4 \mathrm{H}, \mathrm{H}-9 \mathrm{a}^{b}, \mathrm{H}-9 \mathrm{~b}^{b}, \mathrm{H}-9 \mathrm{a}^{c}, \mathrm{H}-9 \mathrm{~b}^{c}\right), 3.32-3.26(2 \mathrm{~s}, 6 \mathrm{H}$, $2 \mathrm{OMe}$ ), 2.76-2.57 (m, $3 \mathrm{H}, \mathrm{H}-3 \mathrm{eq}^{c}, \mathrm{OCH}_{2} \mathrm{CH}_{2} \mathrm{CN}$ ), 2.52 (dd, $1 \mathrm{H}, J_{3 \mathrm{eq}, 4}=5.5 \mathrm{~Hz}, J_{\mathrm{gem}}=13.5 \mathrm{~Hz}, \mathrm{H}-$ $3 \mathrm{eq}^{b}$ ), 2.27-1.93 (m, $35 \mathrm{H}, \mathrm{H}-3 \mathrm{ax}^{c}, \mathrm{H}-6^{C e r}, \mathrm{H}-2^{\text {, Cer }}, 10 \mathrm{Ac}$ ), 1.82 (dd, $1 \mathrm{H}, J_{3 \mathrm{ax}, 4}=11.0 \mathrm{~Hz}, \mathrm{H}-3 \mathrm{ax}^{b}$ ), 1.64-1.58 (m, 2 H, H-3 ${ }^{\text {'Cer }}$ ), 1.40-1.11 (m, 58 H, $29 \mathrm{CH}_{2}$ ), 0.91-0.86 (m, $\left.6 \mathrm{H}, 2 \mathrm{Me}\right) ;{ }^{13} \mathrm{C}$ NMR (125 $\left.\mathrm{MHz}, \mathrm{CDCl}_{3}\right) \delta 171.0,170.4,170.1,170.0,169.6,169.4,169.3,167.8,167.6,138.5,133.4,129.8$, $129.8,128.6,116.7,98.4,95.3,73.0,70.8,69.5,69.0,68.0,67.6,62.8,59.2,59.1,53.3,49.3,37.7$, $36.6,32.3,31.9,29.8,29.7,29.7,29.6,29.5,29.5,29.4,28.9,25.7,22.7,21.2,20.9,20.9,20.8,20.7$, 20.6, 20.5, 14.1; ${ }^{31} \mathrm{P}$ NMR $\left(202 \mathrm{MHz}, \mathrm{CDCl}_{3}\right) \delta-2.31$; HRMS (ESI) $\mathrm{m} / z$ : found $[\mathrm{M}+\mathrm{Na}]^{+} 2101.9897$, $\mathrm{C}_{101} \mathrm{H}_{155} \mathrm{~N}_{4} \mathrm{O}_{39} \mathrm{P}$ calcd for $[\mathrm{M}+\mathrm{Na}]^{+} 2101.9898$. 
1-D-4,5,6-O-Acetyl- \{4',7', 8'-tri- $O$-acetyl-5'-acetoxyacetamido-3',5'-dideoxy-9'-O-methyl-5[methyl 4,7,8-tri- $O$-acetyl-3,5-dideoxy-9-O-methyl-5-(methyl 5-acetoxyacetamido-4,7,8-tri- $O$ acetyl-3,5-dideoxy-9-O-methyl-D-glycero- $\alpha$-D-galacto-2-nonulopyranosylonat)oxyacetamido-Dglycero- $\alpha$-D-galacto-2-nonulopyranosylonat]oxyacetamido-D-glycero- $\alpha$-D-galacto-2'nonulopyranosylono-1',2-lactone $\}-1-O-\{[(2 S, 3 R, 4 E)$-3-O-benzoyl-2-O-tetracosamido-hexadec-4ene-1,3-diol-1-yl] (2-cyanoethoxy)phosphoryl\}-myo-inositol (38)

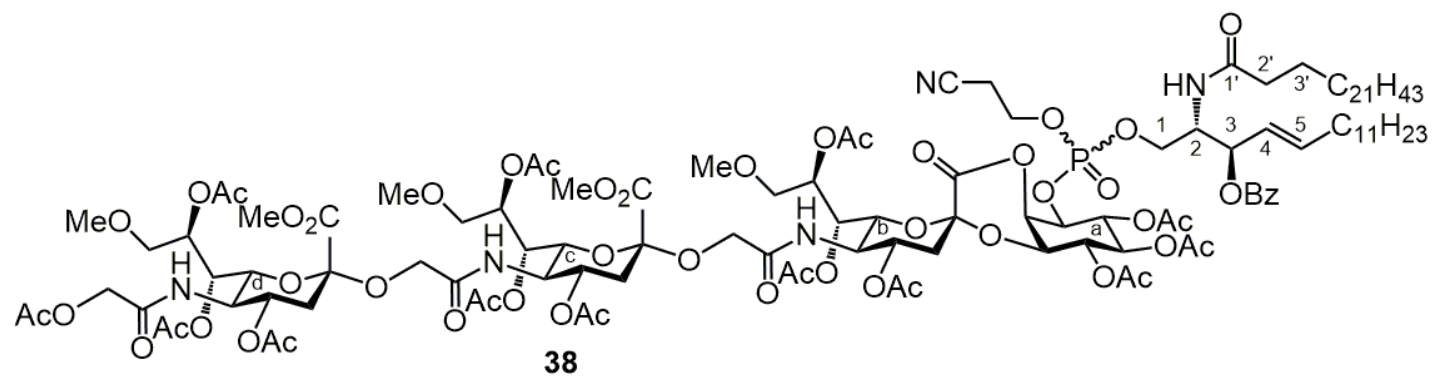

For preparation of 6: To a solution of $34(44 \mathrm{mg}, 60.0 \mu \mathrm{mol})$ and DIEA (21 $\mu \mathrm{L}, 120 \mu \mathrm{mol})$ in $\mathrm{CH}_{2} \mathrm{Cl}_{2}$ $(600 \mu \mathrm{L})$ was added 2-cyanoethyl $N, N$-diisopropylchlorophosphoramidite $35(20 \mu \mathrm{L}, 90.0 \mu \mathrm{mol})$ at rt. After stirring for $10 \mathrm{~min}$, the mixture was concentrated to a quarter of the original volume and passed through short silica gel column chromatography (15:1 $n$-hexane-EtOAc containing 3\% triethylamine) to give 6, which was immediately used for the following coupling reaction due to its instability. To a solution of $32(34 \mathrm{mg}, 20.0 \mu \mathrm{mol})$ in $\mathrm{CH}_{2} \mathrm{Cl}_{2}-\mathrm{MeCN}(1: 1,1.0 \mathrm{~mL})$ were added $1 H$-tetrazole $(8.5 \mathrm{mg}, 12.0 \mu \mathrm{mol})$ and $4 \AA$ molecular sieves $(200 \mathrm{mg})$ at $\mathrm{rt}$ and the mixture was stirred for $30 \mathrm{~min}$ at $\mathrm{rt}$. After addition of a solution of the crude 6 in $\mathrm{CH}_{2} \mathrm{Cl}_{2}-\mathrm{MeCN}(1: 1,1.0 \mathrm{~mL})$ and stirring for additional $10 \mathrm{~min}$ at $\mathrm{rt}$, the completion of the reaction was confirmed by TLC (1:1 $\mathrm{CHCl}_{3}$-acetone). The reaction mixture then was treated with $5.5 \mathrm{M}$ solution of TBHP in decane $(18 \mu \mathrm{L}, 100$ $\mu \mathrm{mol})$. After stirring for extra $10 \mathrm{~min}$, the completion of the reaction was confirmed by TLC (2:1 $\mathrm{CHCl}_{3}$-acetone). The reaction mixture was diluted with $\mathrm{CHCl}_{3}$, washed with brine. The organic layer was dried over $\mathrm{Na}_{2} \mathrm{SO}_{4}$, and concentrated. The resulting residue was purified by silica gel column chromatography (1:1 $\mathrm{CHCl}_{3}$-acetone) to give $\mathbf{3 8}(34 \mathrm{mg}, 67 \%$, diastereomeric mixture) as a white amorphous solid; ${ }^{1} \mathrm{H}$ NMR $\left(500 \mathrm{MHz}, \mathrm{CDCl}_{3}\right) \delta 8.09-7.45(\mathrm{~m}, 5 \mathrm{H}, \mathrm{Ph}), 6.40\left(\mathrm{~d}, 1 \mathrm{H}, J_{2, \mathrm{NH}}=9.5 \mathrm{~Hz}\right.$, $\mathrm{NHCO}^{\mathrm{Cer}}$ ), 6.24-5.84 (m, $4 \mathrm{H}, \mathrm{NH}^{b}, \mathrm{NH}^{c}, \mathrm{NH}^{d}, \mathrm{H}-5^{\mathrm{Cer}}$ ), 4.79 (m, $\left.1 \mathrm{H}, \mathrm{H}-1^{a}\right), 4.47$ (dd, $1 \mathrm{H}, J_{3,4}=8.0$ $\mathrm{Hz}, \mathrm{H}-3^{a}$ ), 3.56 (t, $1 \mathrm{H}, J_{1,2}=J_{2,3}=2.5 \mathrm{~Hz}, \mathrm{H}-2^{a}$ ), 2.79-2.50 (m, $5 \mathrm{H}, \mathrm{H}-3 \mathrm{eq}^{b}, \mathrm{H}-3 \mathrm{eq}^{c}, \mathrm{H}-3 \mathrm{eq}^{d}$, $\mathrm{OCH}_{2} \mathrm{CH}_{2} \mathrm{CN}$ ), 2.29-1.77 (m, 46 H, H-3ax ${ }^{b}, \mathrm{H}-3 \mathrm{ax}^{c}, \mathrm{H}-3 \mathrm{ax}^{d}, \mathrm{H}-6^{\mathrm{Cer}}, \mathrm{H}-2{ }^{\text {, }}{ }^{\mathrm{Cer}}, 13 \mathrm{Ac}$ ), 1.64-1.57 (m, 2 H, H-3 ${ }^{\text {Cer }}$ ), $1.40-1.20$ (m, 58 H, $29 \mathrm{CH}_{2}$ ), 0.90-0.86 (m, $6 \mathrm{H}, 2 \mathrm{Me}$ ); ${ }^{13} \mathrm{C} \mathrm{NMR}\left(125 \mathrm{MHz}, \mathrm{CDCl}_{3}\right) \delta$ 173.2, 173.0, 171.2, 171.0, 170.5, 170.4, 170.3, 170.1 170.1, 170.0, 170.0, 169.6, 169.4, 169.4, 169.3, 169.2, 168.8, 168.7, 168.5, 167.8, 167.6, 166.0, 165.4, 162.9, 162.5, 138.4, 138.1, 133.4, 129.8, 129.8 , 129.6, 128.7, 128.6, 124.7, 124.3, 116.7, 116.4, 98.4, 98.4, 95.5, 95.3, 73.3, 73.2, 73.1, 72.9, 72.8, $72.2,71.0,70.9,70.7,69.9,69.7,69.6,69.4,69.1,69.0,68.8,68.6,68.4,68.1,67.6,67.4,63.9,63.8$, 
62.8, 59.2, 59.1, 59.1, 59.0, 53.3, 53.2, 49.2, 48.7, 48.5, 37.9, 37.7, 36.6, 36.5, 32.3, 31.9, 29.7, 29.7, 29.6, 29.6, 29.6, 29.6, 29.5, 29.5, 29.5, 29.4, 29.3, 29.3, 28.8, 25.7, 22.7, 21.2, 21.2, 20.9, 20.9, 20.9, 20.8, 20.8, 20.7, 20.6, 20.6, 20.5, 20.4, 19.6, 19.3, 14.1; HRMS (ESI) $m / z$ : found $[\mathrm{M}+2 \mathrm{Na}]^{2+}$ 1293.0664, $\mathrm{C}_{120} \mathrm{H}_{182} \mathrm{~N}_{5} \mathrm{O}_{51} \mathrm{P}$ calcd for $[\mathrm{M}+2 \mathrm{Na}]^{2+} 1293.0662$.

Sialyl inositol phosphospingolipid CJP-2 (1)

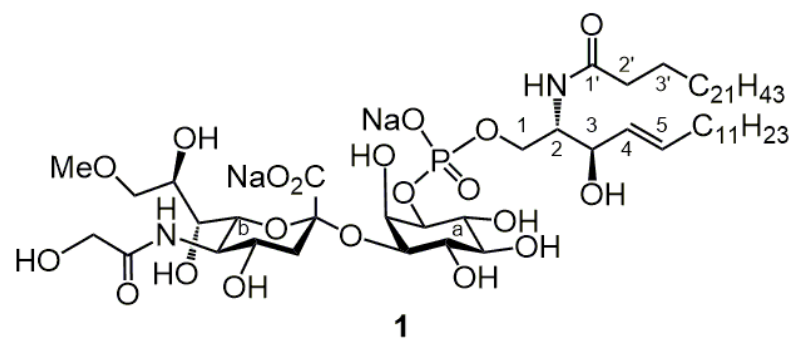

To a solution of 36 (49 mg, $39.5 \mu \mathrm{mol})$ in $\mathrm{MeOH}-\mathrm{THF}-\mathrm{H}_{2} \mathrm{O}(2: 1: 1,2.0 \mathrm{~mL})$ was added $1.0 \mathrm{M} \mathrm{MeOH}$ solution of $\mathrm{NaOMe}(306 \mu \mathrm{L}, 306 \mu \mathrm{mol})$ at $0{ }^{\circ} \mathrm{C}$. After stirring for $5 \mathrm{~h}$ at $\mathrm{rt}$, the completion of the reaction was confirmed by TLC $\left(5: 4: 1 \mathrm{CHCl}_{3}-\mathrm{MeOH}-\mathrm{H}_{2} \mathrm{O}\right)$. The reaction mixture was purified by column chromatography on Sephadex LH-20 (5:4:1 $\left.\mathrm{CHCl}_{3}-\mathrm{MeOH}-\mathrm{H}_{2} \mathrm{O}\right)$ and Iatrobeads 6RS-8060 $\left(10: 6: 1 \rightarrow 12: 8: 1 \mathrm{CHCl}_{3}-\mathrm{MeOH}-\mathrm{H}_{2} \mathrm{O}\right)$ to give $\mathbf{1}(22 \mathrm{mg}, 88 \%)$ as a white amorphous solid; $[\alpha]_{\mathrm{D}}+3.7^{\circ}$ (c 1.0, 5:4:1 CHCl $\left.-\mathrm{MeOH}-\mathrm{H}_{2} \mathrm{O}\right) ;{ }^{1} \mathrm{H}$ NMR $\left(500 \mathrm{MHz}, 5: 4: 1 \mathrm{CDCl}_{3}-\mathrm{CD}_{3} \mathrm{OD}-\mathrm{D}_{2} \mathrm{O}\right) \delta 5.71(\mathrm{~m}, 1 \mathrm{H}$, $\mathrm{H}-5^{\mathrm{Cer}}$ ), 5.42 (dd, $1 \mathrm{H}, J_{3,4}=8.0 \mathrm{~Hz}, J_{4,5}=15.0 \mathrm{~Hz}, \mathrm{H}-4^{\mathrm{Cer}}$ ), 3.40 (s, $\left.3 \mathrm{H}, \mathrm{OMe}\right), 2.80$ (d, $1 \mathrm{H}, J=11.0$ Hz, H-3eq $\left.{ }^{b}\right), 2.19$ (m, 2 H, H-2 ${ }^{C e r}$ ), 2.01 (m, 2 H, H-6 $\left.{ }^{C e r}\right), 1.78$ (t, 1 H, $J=9.0$ Hz, H-3ax $\left.{ }^{b}\right), 1.58$ (m, $2 \mathrm{H}, \mathrm{H}-3{ }^{\text {'Cer }}$ ), 1.48-1.15 (m, $58 \mathrm{H}, 29 \mathrm{CH}_{2}$ ), 0.93-0.86 (m, $6 \mathrm{H}, 2 \mathrm{Me}$ ); ${ }^{13} \mathrm{C}$ NMR (125 MHz, 5:4:1 $\left.\mathrm{CDCl}_{3}-\mathrm{CD}_{3} \mathrm{OD}-\mathrm{D}_{2} \mathrm{O}\right) \delta 177.3,136.2,130.5,79.1,75.6,75.1,74.1,72.5,72.2,71.7,69.9,69.2,60.1$, 53.3, 37.8, 33.8, 33.3, 33.2, 31.2, 31.1, 31.1, 31.1, 31.0, 31.0, 31.0, 30.8, 30.7, 30.7, 30.7, 27.5, 24.0, 24.0, 15.3, 15.2; ${ }^{31} \mathrm{P}$ NMR (202 MHz, 5:4:1 $\mathrm{CDCl}_{3}-\mathrm{CD}_{3} \mathrm{OD}-\mathrm{D}_{2} \mathrm{O}$ ) $\delta-0.89$; HRMS (ESI) $\mathrm{m} / z$ : found $[\mathrm{M}-2 \mathrm{Na}]^{2-} 591.3581, \mathrm{C}_{58} \mathrm{H}_{107} \mathrm{~N}_{2} \mathrm{Na}_{2} \mathrm{O}_{20} \mathrm{P}$ calcd for $[\mathrm{M}-2 \mathrm{Na}]^{2-} 591.3583$.

Sialyl inositol phosphospingolipid CJP-3 (2)

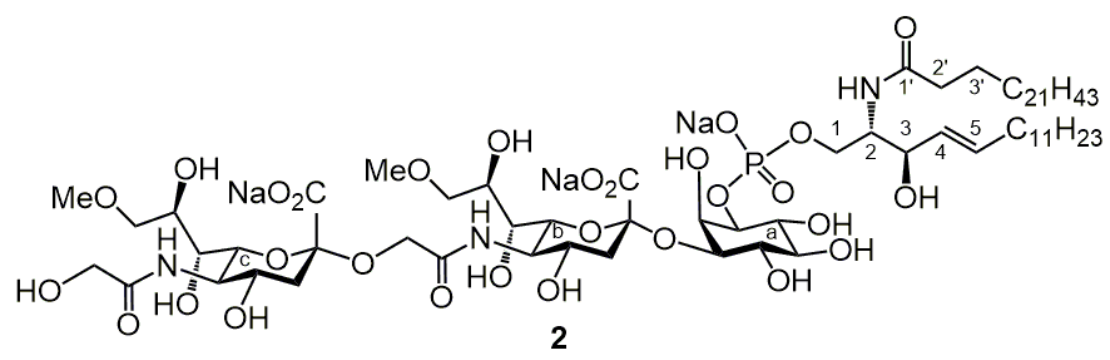

To a solution of $37(38 \mathrm{mg}, 18.3 \mu \mathrm{mol})$ in $\mathrm{MeOH}-\mathrm{THF}-\mathrm{H}_{2} \mathrm{O}(2: 1: 1,1.8 \mathrm{~mL})$ was added $1.0 \mathrm{M} \mathrm{MeOH}$ solution of $\mathrm{NaOMe}(274 \mu \mathrm{L}, 274 \mu \mathrm{mol})$ at $0{ }^{\circ} \mathrm{C}$. After stirring for $20 \mathrm{~h}$ at rt, the completion of the reaction was confirmed by TLC $\left(5: 4: 1 \mathrm{CHCl}_{3}-\mathrm{MeOH}-\mathrm{H}_{2} \mathrm{O}\right)$. The reaction mixture was purified by 
column chromatography on Sephadex LH-20 (5:4:1 $\left.\mathrm{CHCl}_{3}-\mathrm{MeOH}-\mathrm{H}_{2} \mathrm{O}\right)$ and Iatrobeads 6RS-8060 $\left(10: 6: 1 \rightarrow 12: 8: 1 \mathrm{CHCl}_{3}-\mathrm{MeOH}-\mathrm{H}_{2} \mathrm{O}\right)$ to give $2(18 \mathrm{mg}, 64 \%)$ as a white amorphous solid; $[\alpha]_{\mathrm{D}}-1.0^{\circ}$ (c 1.0, 5:4:1 $\left.\mathrm{CHCl}_{3}-\mathrm{MeOH}-\mathrm{H}_{2} \mathrm{O}\right) ;{ }^{1} \mathrm{H}$ NMR $\left(500 \mathrm{MHz}, 5: 4: 1 \mathrm{CDCl}_{3}-\mathrm{CD}_{3} \mathrm{OD}-\mathrm{D}_{2} \mathrm{O}\right) \delta 5.71(\mathrm{~m}, 1 \mathrm{H}$, $\left.\mathrm{H}-5^{\mathrm{Cer}}\right), 5.40\left(\mathrm{dd}, 1 \mathrm{H}, J_{3,4}=8.0 \mathrm{~Hz}, J_{4,5}=15.5 \mathrm{~Hz}, \mathrm{H}-4^{\mathrm{Cer}}\right), 3.40(2 \mathrm{~s}, 6 \mathrm{H}, 2 \mathrm{OMe}), 2.80(\mathrm{~m}, 2 \mathrm{H}, \mathrm{H}-$ $\left.3 \mathrm{eq}^{b}, \mathrm{H}-3 \mathrm{eq}^{c}\right), 2.20$ (m, $2 \mathrm{H}, \mathrm{H}-2^{\text {Cer }}$ ), 2.02 (m, $2 \mathrm{H}, \mathrm{H}-6^{C e r}$ ), 1.78 (m, $2 \mathrm{H}, \mathrm{H}-3 \mathrm{ax}^{b}, \mathrm{H}-3 \mathrm{ax}^{c}$ ), 1.56 (m, $2 \mathrm{H}, \mathrm{H}-3{ }^{\text {'Cer }}$ ), $1.42-1.15$ (m, $58 \mathrm{H}, 29 \mathrm{CH}_{2}$ ), 0.93-0.86 (m, $\left.6 \mathrm{H}, 2 \mathrm{Me}\right) ;{ }^{13} \mathrm{C}$ NMR (125 MHz, 5:4:1 $\left.\mathrm{CDCl}_{3}-\mathrm{CD}_{3} \mathrm{OD}-\mathrm{D}_{2} \mathrm{O}\right) \delta 177.3,176.4,136.1,130.5,101.7,79.1,75.5,75.0,74.1,72.2,71.5,69.9$, 69.2, 69.1, 62.7, 60.0, 53.3, 37.8, 33.8, 33.3, 33.2, 31.2, 31.1, 31.1, 31.0, 31.0, 30.9, 30.8, 30.7, 30.7, 30.7, 27.5, 24.0, 24.0, 15.2, 15.2; ${ }^{31} \mathrm{P}$ NMR (202 MHz, 5:4:1 $\left.\mathrm{CDCl}_{3}-\mathrm{CD}_{3} \mathrm{OD}-\mathrm{D}_{2} \mathrm{O}\right) \delta$ 0.21; HRMS (ESI) $m / z$ : found $[\mathrm{M}-2 \mathrm{Na}]^{2-} 762.9021, \mathrm{C}_{70} \mathrm{H}_{125} \mathrm{~N}_{3} \mathrm{Na}_{3} \mathrm{O}_{29} \mathrm{P}$ calcd for $[\mathrm{M}-2 \mathrm{Na}]^{2-} 762.9023$.

Sialyl inositol phosphospingolipid CJP-4 (3)

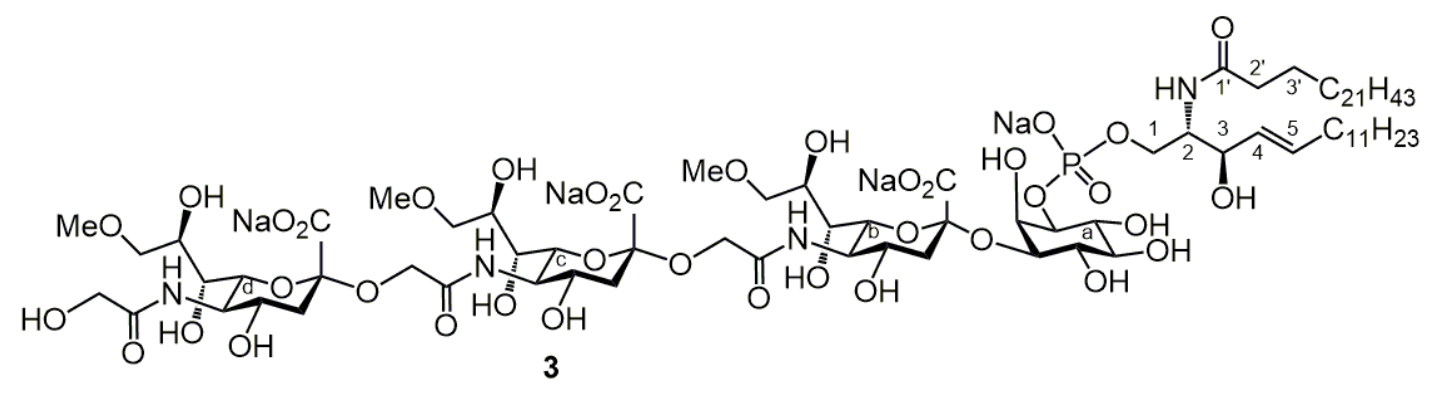

To a solution of $\mathbf{3 8}(33 \mathrm{mg}, 13.0 \mu \mathrm{mol})$ in $\mathrm{MeOH}-\mathrm{THF}-\mathrm{H}_{2} \mathrm{O}(2: 1: 1,1.3 \mathrm{~mL})$ was added $1.0 \mathrm{M} \mathrm{MeOH}$ solution of $\mathrm{NaOMe}(195 \mu \mathrm{L}, 195 \mu \mathrm{mol})$ at $0{ }^{\circ} \mathrm{C}$. After stirring for $20 \mathrm{~h}$ at rt, the completion of the reaction was confirmed by TLC $\left(5: 4: 1 \mathrm{CHCl}_{3}-\mathrm{MeOH}-\mathrm{H}_{2} \mathrm{O}\right)$. The reaction mixture was purified by column chromatography on Sephadex LH-20 (5:4:1 $\left.\mathrm{CHCl}_{3}-\mathrm{MeOH}-\mathrm{H}_{2} \mathrm{O}\right)$ and Iatrobeads 6RS-8060 $\left(8: 5: 1 \rightarrow 5: 4: 1 \mathrm{CHCl}_{3}-\mathrm{MeOH}-\mathrm{H}_{2} \mathrm{O}\right)$ to give $3(12 \mathrm{mg}, 47 \%)$ as a white amorphous solid; $[\alpha]_{\mathrm{D}}-6.2 \circ$ (c 1.0, 5:4:1 CHCl $\left.3-\mathrm{MeOH}-\mathrm{H}_{2} \mathrm{O}\right) ;{ }^{1} \mathrm{H}$ NMR $\left(500 \mathrm{MHz}, 5: 4: 1 \mathrm{CDCl}_{3}-\mathrm{CD}_{3} \mathrm{OD}-\mathrm{D}_{2} \mathrm{O}\right) \delta 5.70(\mathrm{~m}, 1 \mathrm{H}$, $\left.\mathrm{H}-5^{\mathrm{Cer}}\right), 5.40\left(\mathrm{dd}, 1 \mathrm{H}, J_{3,4}=8.0 \mathrm{~Hz}, J_{4,5}=15.0 \mathrm{~Hz}, \mathrm{H}-4^{\mathrm{Cer}}\right), 3.39(3 \mathrm{~s}, 9 \mathrm{H}, 3 \mathrm{OMe}), 2.78(\mathrm{~m}, 3 \mathrm{H}, \mathrm{H}-$ $3 \mathrm{eq}^{b}, \mathrm{H}-3 \mathrm{eq}^{c}, \mathrm{H}-3 \mathrm{eq}^{d}$ ), 2.18 (m, 2 H, H-2 ${ }^{\text {, Cer }}$ ), 2.00 (m, 2 H, H-6 ${ }^{C e r}$ ), 1.76 (m, 3 H, H-3ax ${ }^{b}, \mathrm{H}-3 \mathrm{ax}^{c}, \mathrm{H}-$ $\left.3 \mathrm{ax}^{d}\right), 1.55$ (m, $\left.2 \mathrm{H}, \mathrm{H}-3{ }^{\circ}{ }^{C e r}\right), 1.42-1.15\left(\mathrm{~m}, 58 \mathrm{H}, 29 \mathrm{CH}_{2}\right), 0.93-0.86(\mathrm{~m}, 6 \mathrm{H}, 2 \mathrm{Me}) ;{ }^{13} \mathrm{C}$ NMR $(125$ $\left.\mathrm{MHz}, 5: 4: 1 \mathrm{CDCl}_{3}-\mathrm{CD}_{3} \mathrm{OD}-\mathrm{D}_{2} \mathrm{O}\right) \delta$ 177.3, 174.6, 136.1, 130.5, 79.4, 75.5, 75.0, 74.1, 72.4, 71.5, 69.9, 69.1, 64.6, 62.7, 60.0, 59.9, 53.4, 53.3, 50.6, 50.3, 37.8, 33.8, 33.3, 33.2, 31.2, 31.1, 31.1, 31.1, 31.0, $30.8,30.7,30.7,30.7,27.5,24.0,24.0,15.2 ;{ }^{31} \mathrm{P} \mathrm{NMR}\left(202 \mathrm{MHz}, 5: 4: 1 \mathrm{CDCl}_{3}-\mathrm{CD}_{3} \mathrm{OD}-\mathrm{D}_{2} \mathrm{O}\right) \delta 0.33$; HRMS (ESI) $m / z$ : found $[\mathrm{M}-2 \mathrm{Na}]^{2-} 934.4463, \mathrm{C}_{82} \mathrm{H}_{143} \mathrm{~N}_{4} \mathrm{Na}_{4} \mathrm{O}_{38} \mathrm{P}$ calcd for $[\mathrm{M}-2 \mathrm{Na}]^{2-} 934.4462$. 


\section{References}

1. (a) Ren, C.-T.; Chen, C.-S.; Wu, S.-H. J. Org. Chem. 2002, 67, 1376-1379. (b) Ando, H.; Koike, Y.; Ishida, H.; Kiso, M. Tetrahedron Lett. 2003, 44, 6883-6886.

2. Koketsu, M.; Fukuta, Y.; Ishihara, H. Tetrahedron Lett. 2001, 42, 6333-6335.

3. Bruzik, K.; Salamonczyk, G. M. Carbohydr. Res. 1989, 195, 67-73.

4. Ong, D. E.; Brady, R. N. J. Lipid Res. 1972, 13, 819-822.

5. Herold, P. Helv. Chim. Acta 1988, 71, 354-362. 


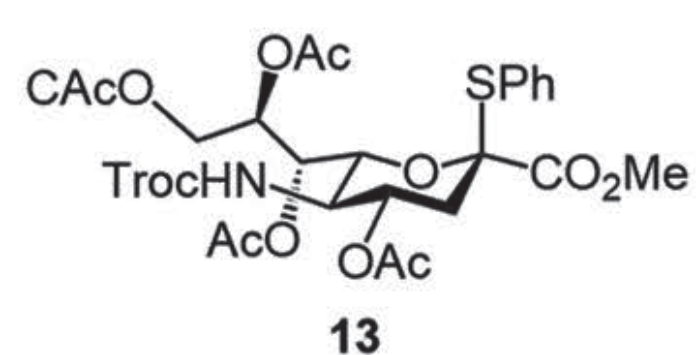

${ }^{1} \mathrm{H} \mathrm{NMR}\left(500 \mathrm{MHz}, \mathrm{CDCl}_{3}\right)$

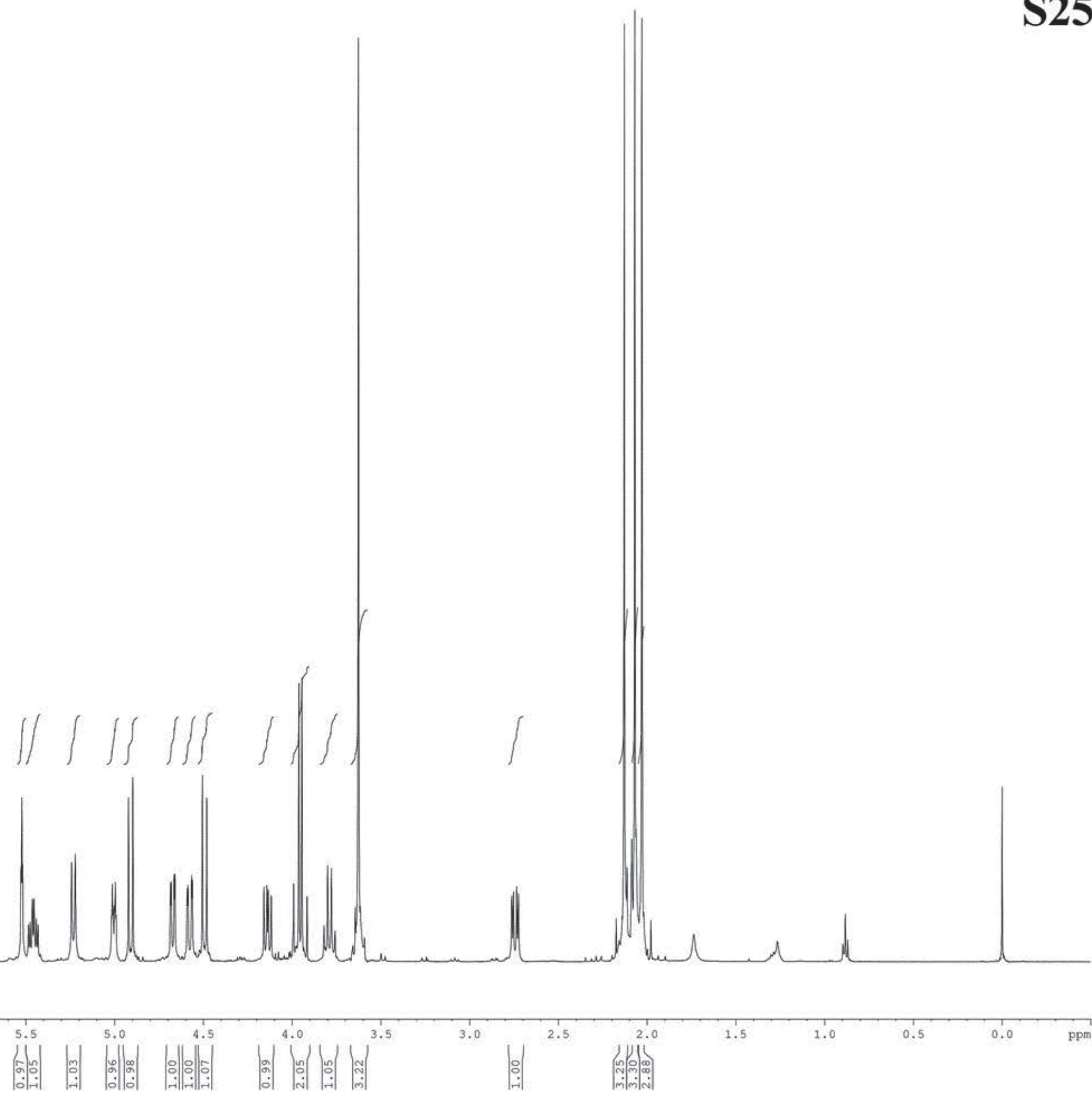




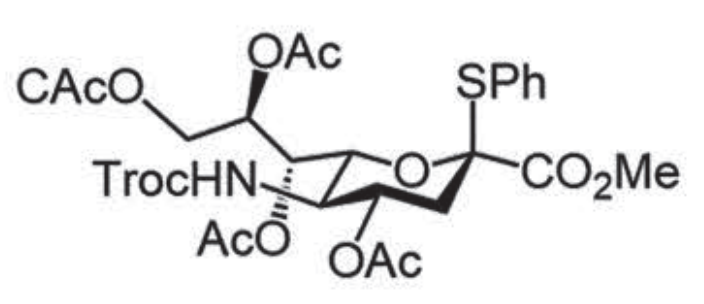

13

${ }^{13} \mathrm{C}$ NMR (125 MHz, $\mathrm{CDCl}_{3}$ ) 


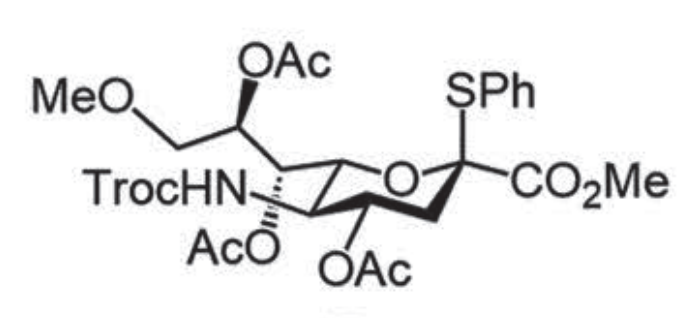

15

${ }^{1} \mathrm{H}$ NMR (500 MHz, $\mathrm{CDCl}_{3}$ )

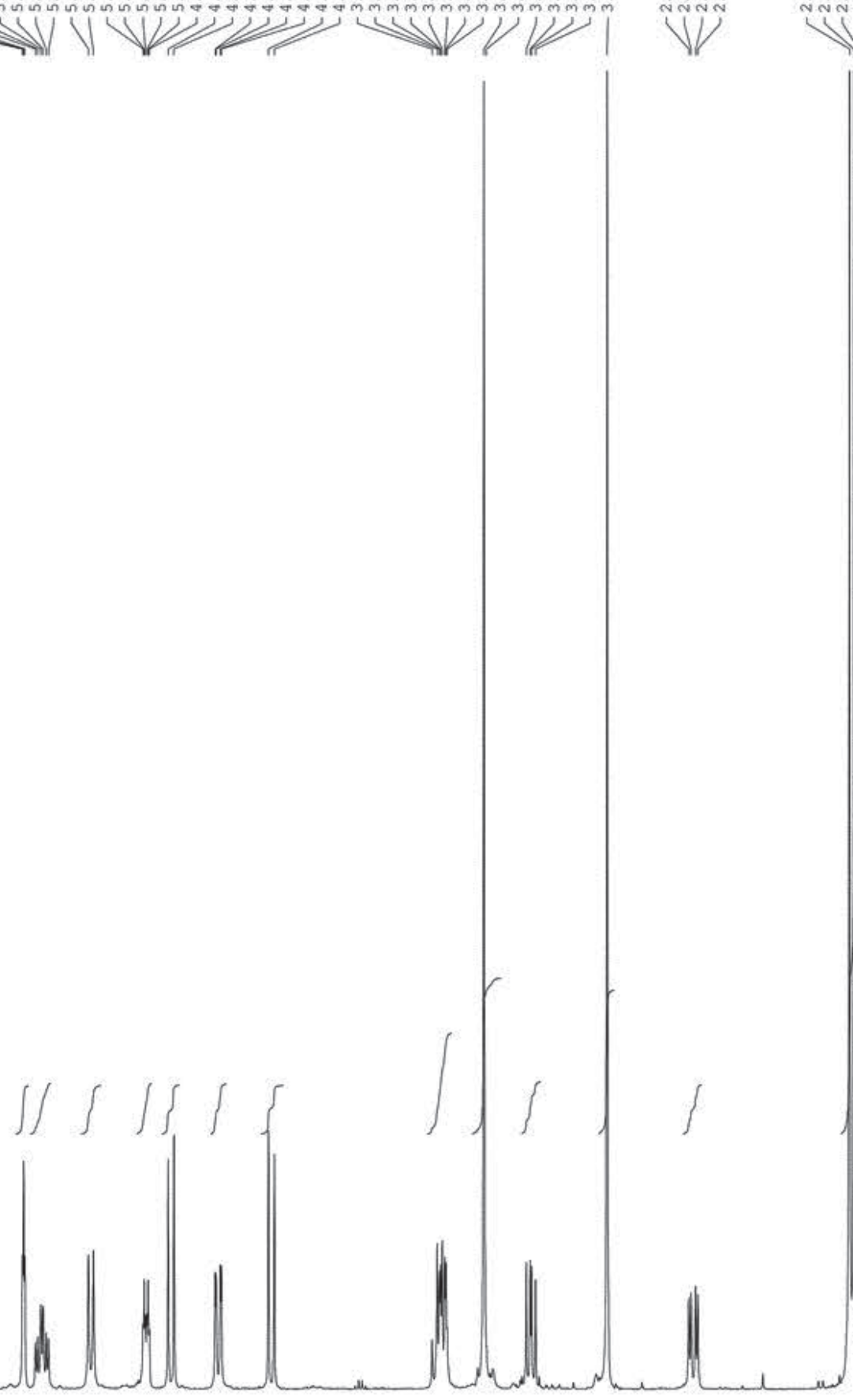

S27 


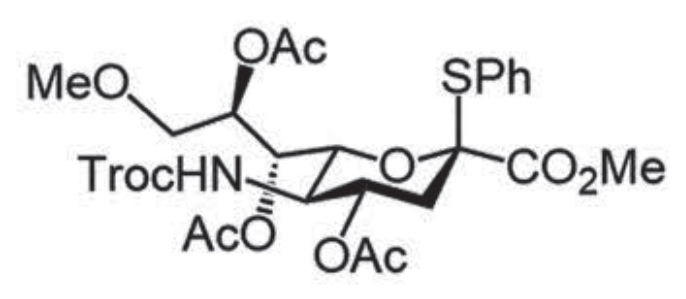

15

${ }^{13} \mathrm{C} \mathrm{NMR}\left(125 \mathrm{MHz}, \mathrm{CDCl}_{3}\right.$ )
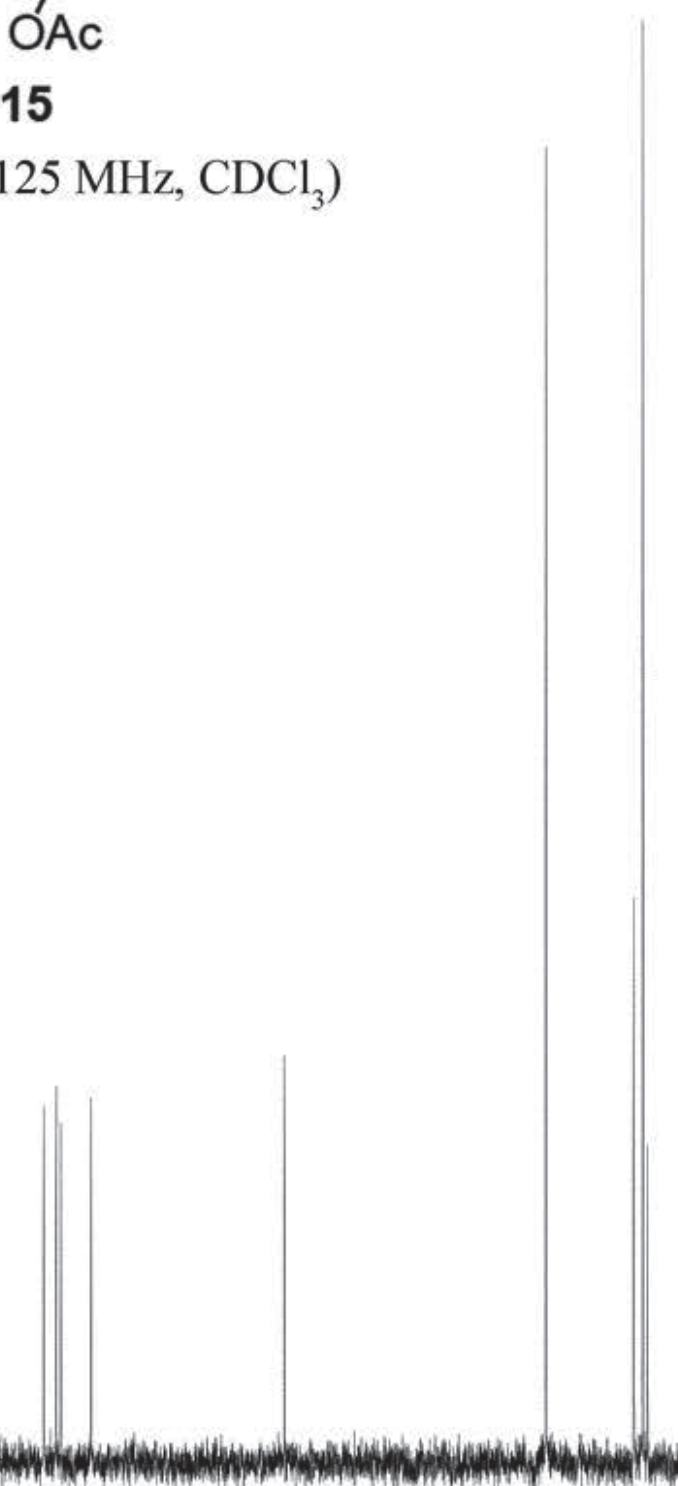


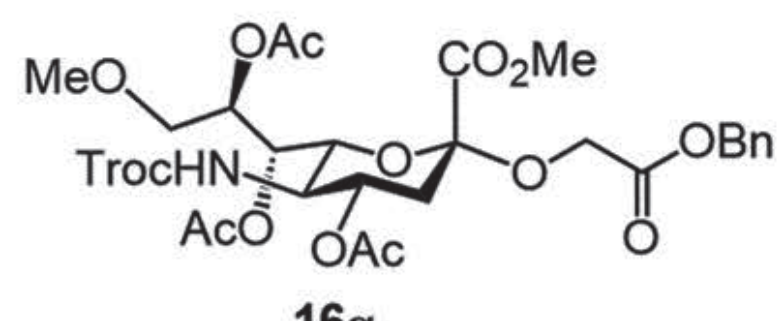

S29

$16 \alpha$

${ }^{1} \mathrm{H} \mathrm{NMR}\left(500 \mathrm{MHz}, \mathrm{CDCl}_{3}\right)$

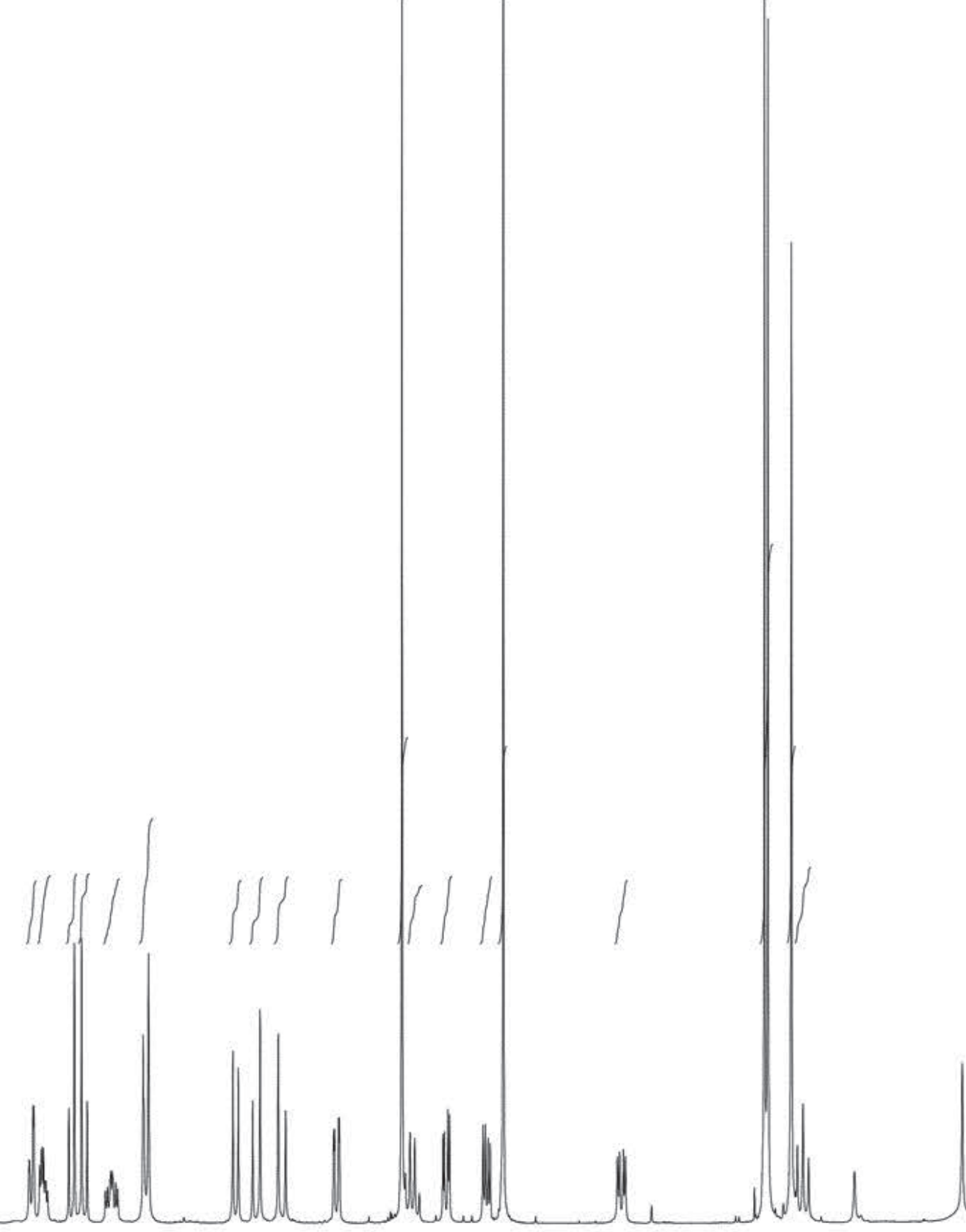




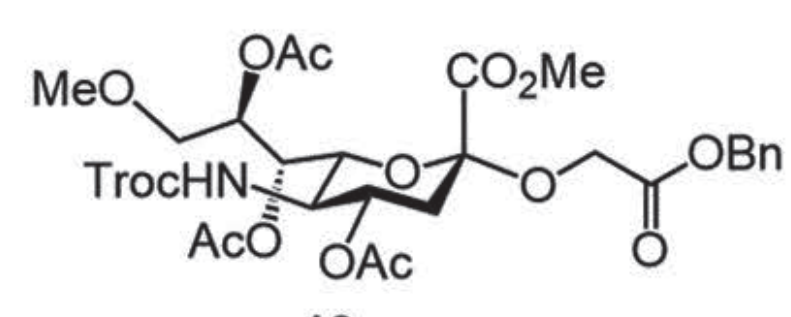

$16 \alpha$

${ }^{13} \mathrm{C} \mathrm{NMR}\left(125 \mathrm{MHz}, \mathrm{CDCl}_{3}\right)$ 


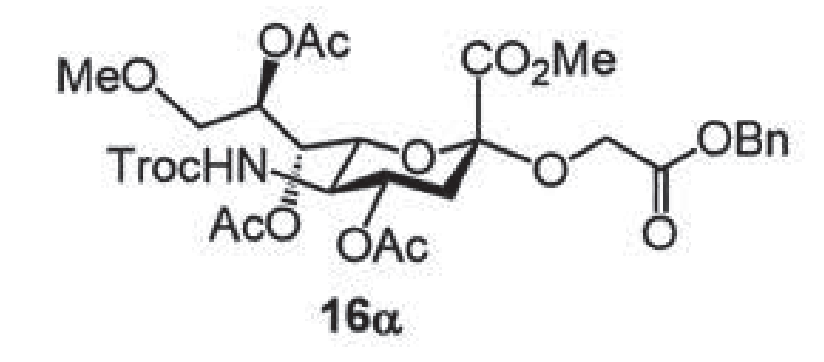

Gated $\left\{{ }^{1} \mathrm{H}\right\}{ }^{13} \mathrm{C}$ NMR $\left(125 \mathrm{MHz}, \mathrm{CDCl}_{3}\right)$
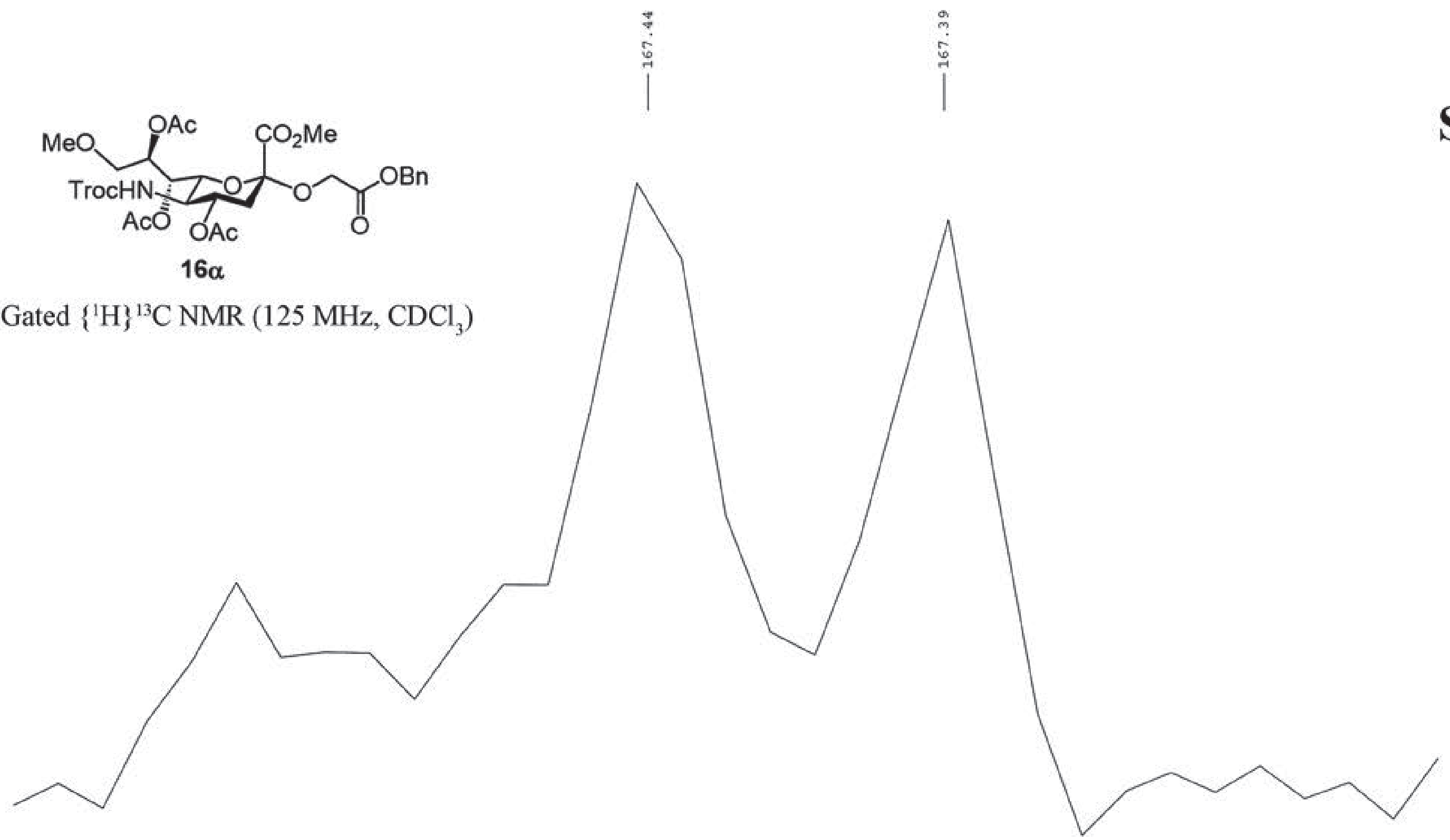


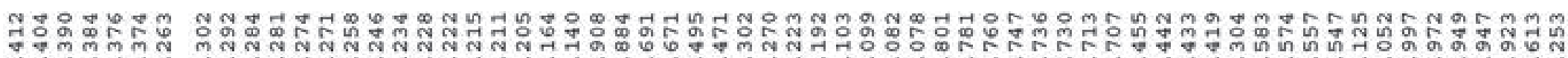

WW
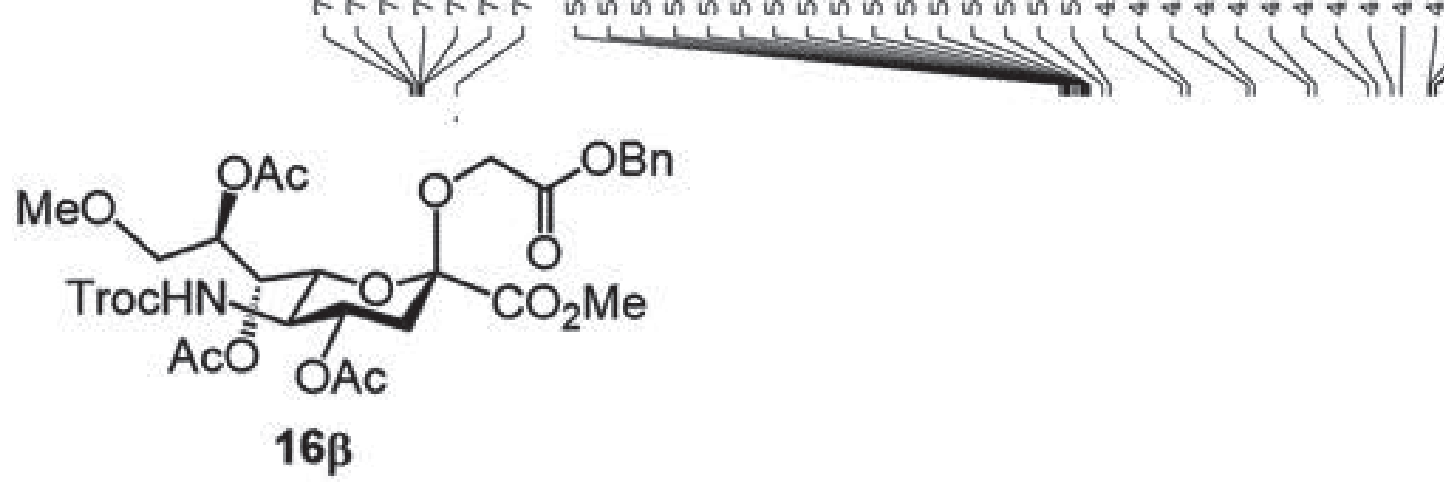

${ }^{1} \mathrm{H} \mathrm{NMR}\left(500 \mathrm{MHz}, \mathrm{CDCl}_{3}\right.$ )

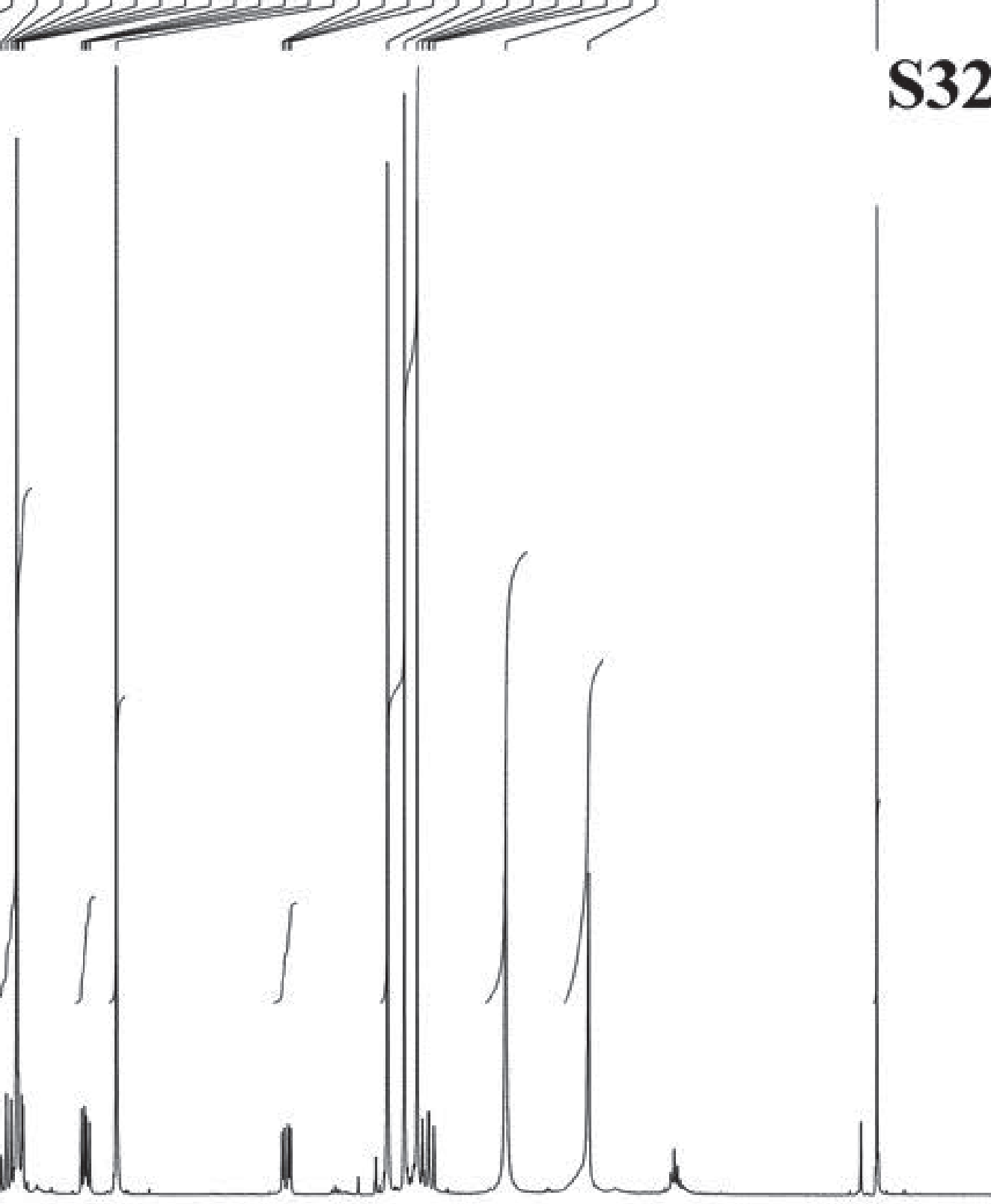


mㅇำ 00000 तन in เก
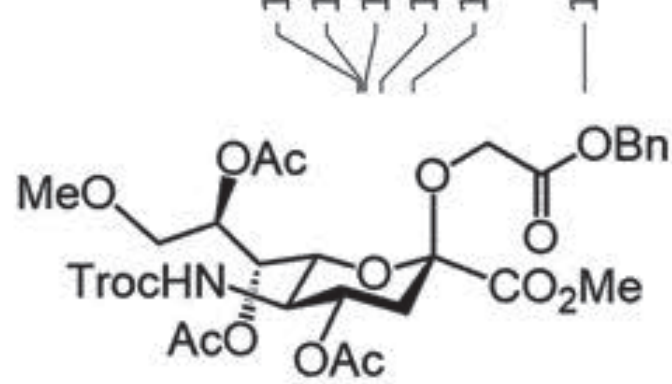

$16 \beta$

${ }^{13} \mathrm{C}$ NMR (125 MHz, $\left.\mathrm{CDCl}_{3}\right)$ $\lim _{\substack{1 \\ 0}}$

in $\infty \infty \infty$ $\mathrm{m} N \mathrm{~N}$

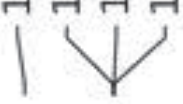

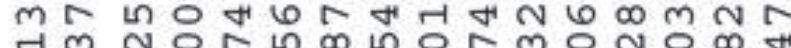

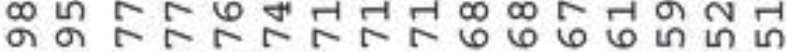
1)
م

त1 0 mN N N
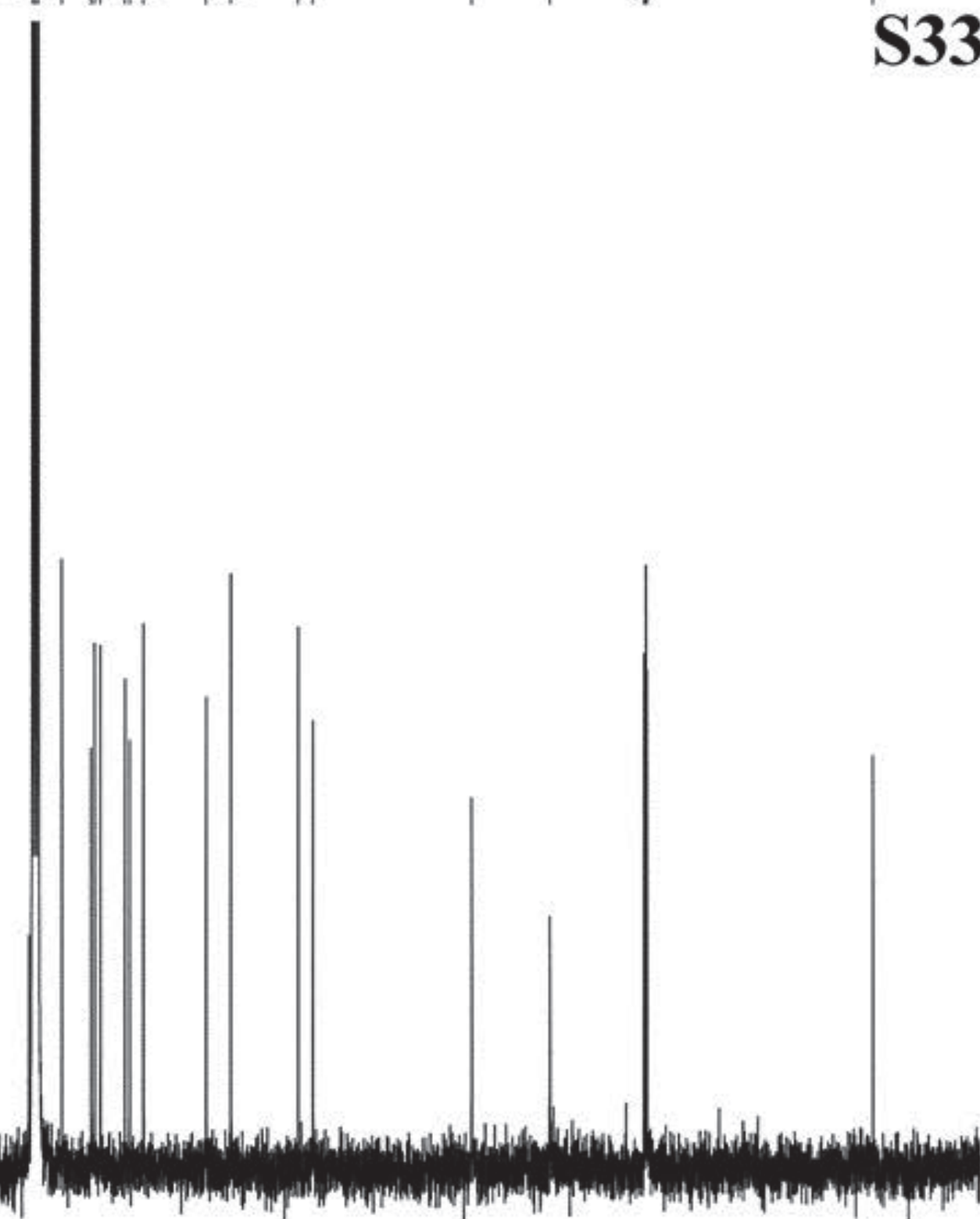
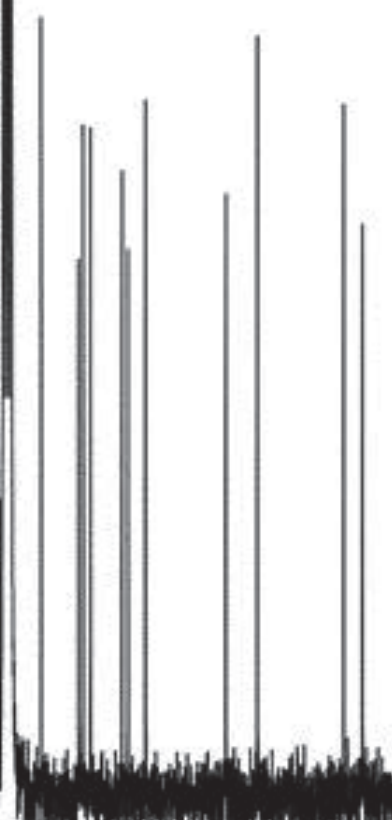


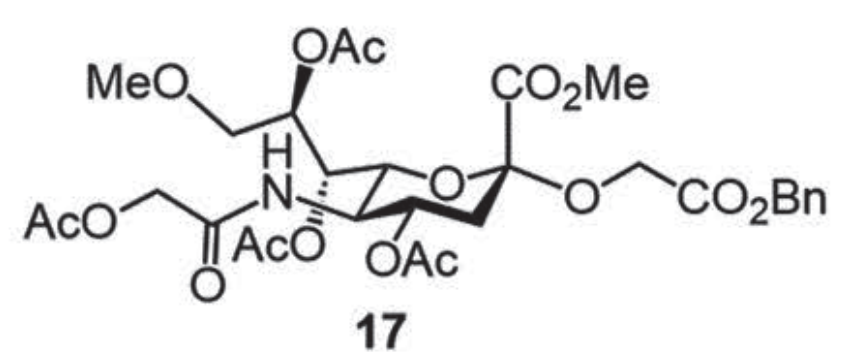

${ }^{1} \mathrm{H} \mathrm{NMR}\left(500 \mathrm{MHz}, \mathrm{CDCl}_{3}\right)$
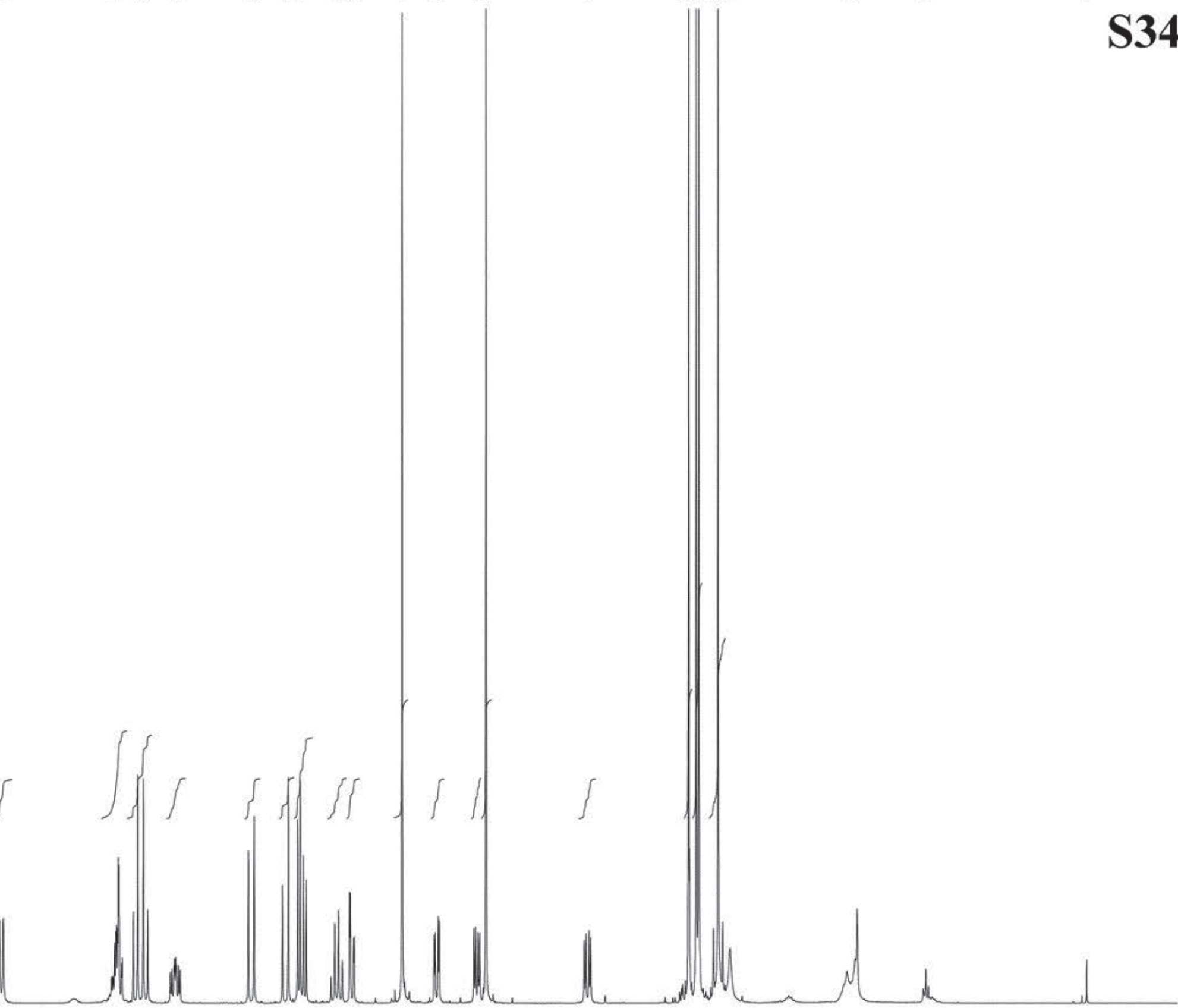


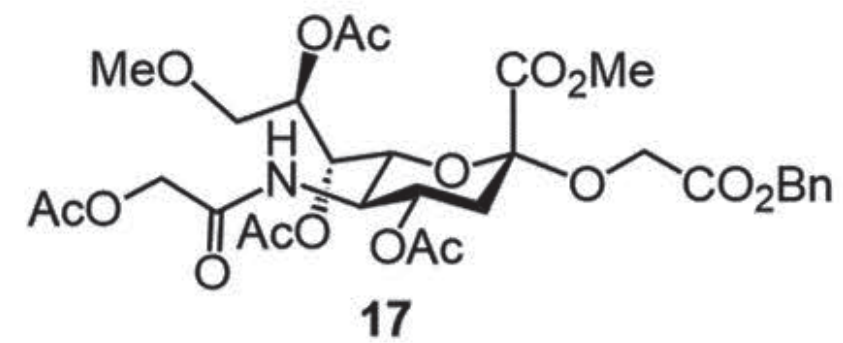

${ }^{13} \mathrm{C}$ NMR (125 MHz, $\mathrm{CDCl}_{3}$ ) 


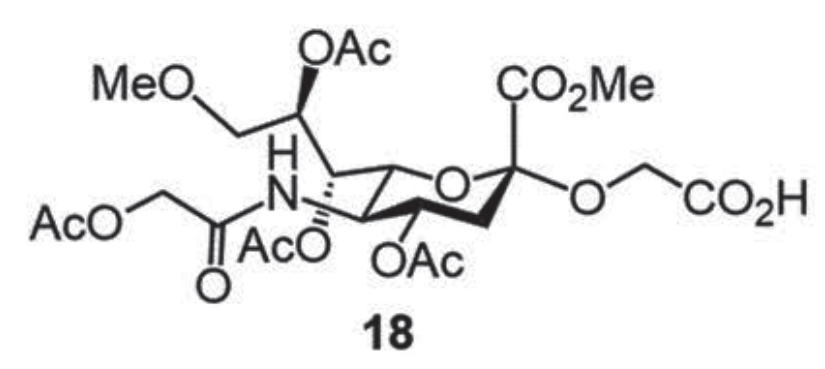

${ }^{1} \mathrm{H}$ NMR (500 MHz, $\mathrm{CDCl}_{3}$ )

S36 

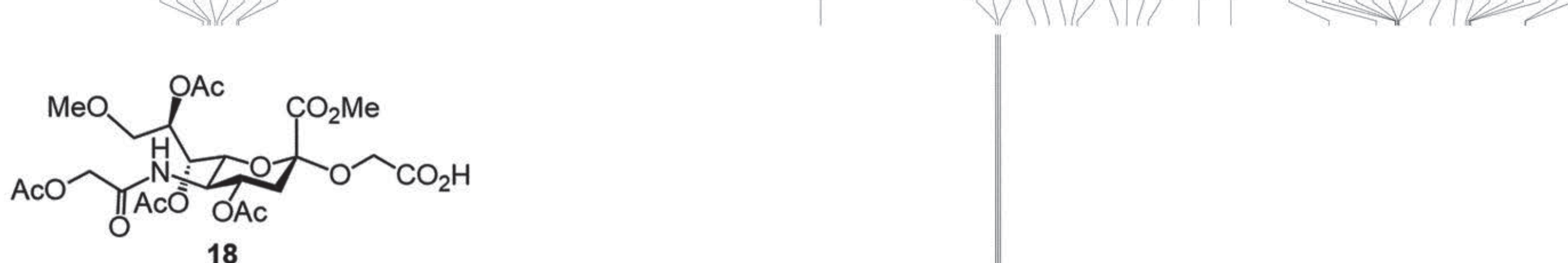

${ }^{13} \mathrm{C}$ NMR (125 MHz, $\left.\mathrm{CDCl}_{3}\right)$ 

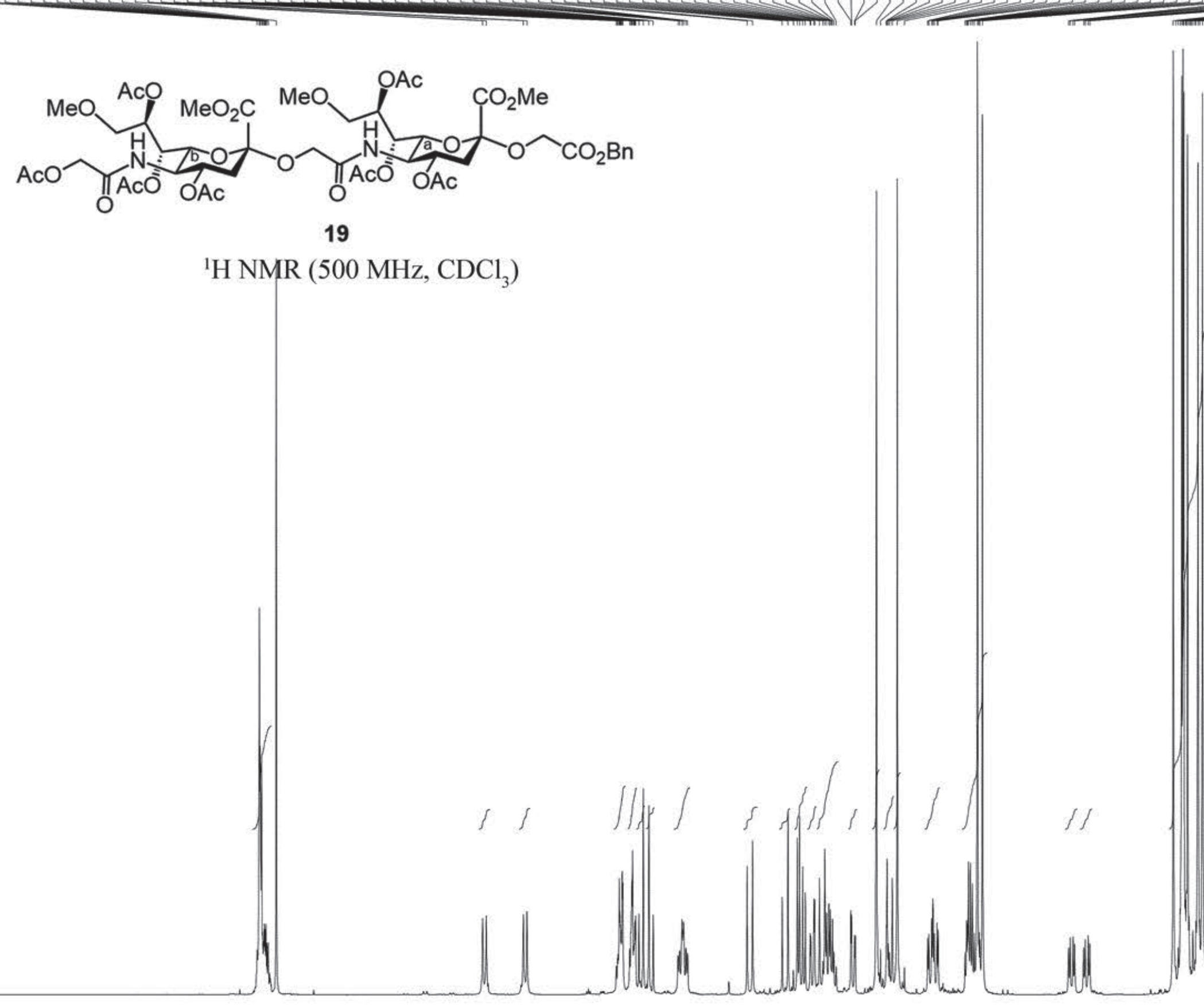

S38

${ }^{1} \mathrm{H} \mathrm{NMR}\left(500 \mathrm{MHz}, \mathrm{CDCl}_{3}\right)$ 

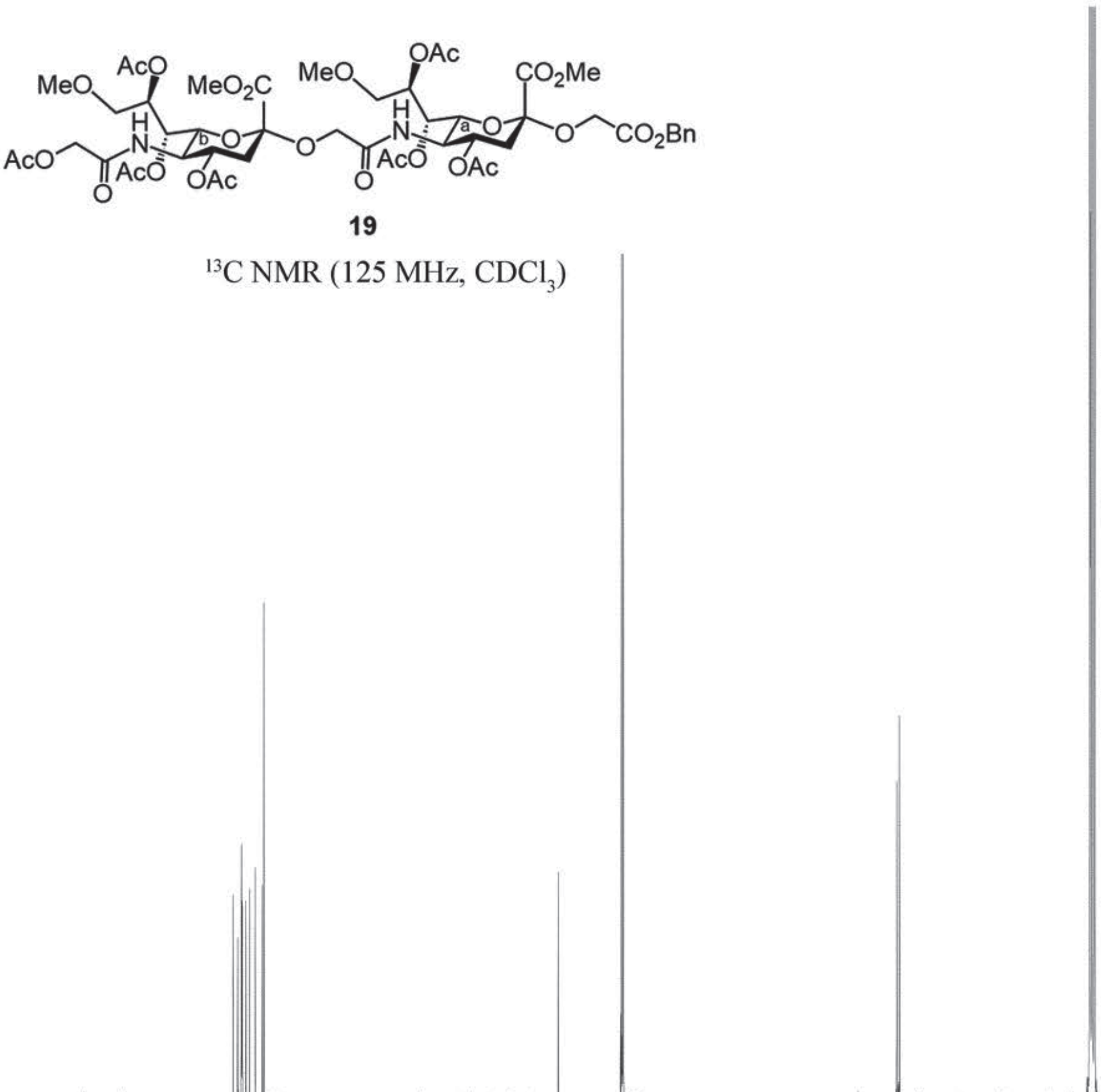

${ }^{13} \mathrm{C}$ NMR (125 MHz, $\left.\mathrm{CDCl}_{3}\right)$ 


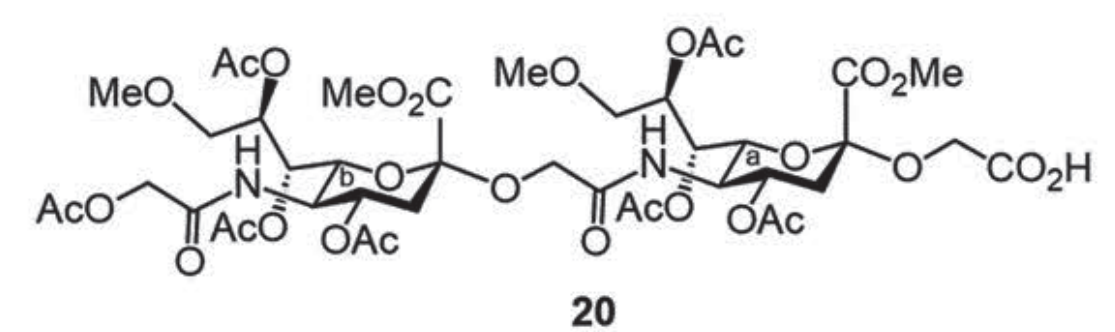

${ }^{1} \mathrm{H}$ NMR (500 MHz, $\mathrm{CDCl}_{3}$ )
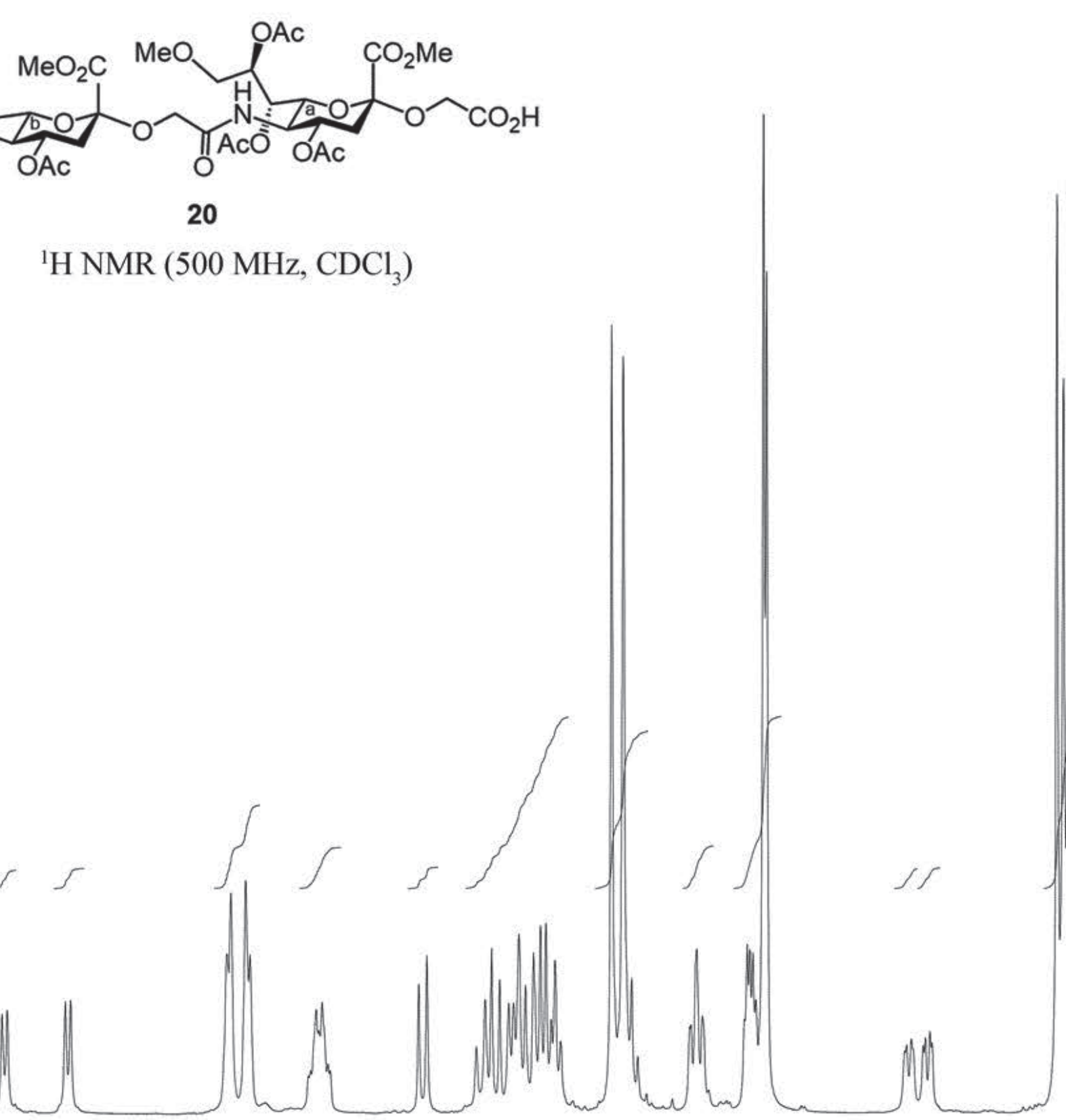

S40 


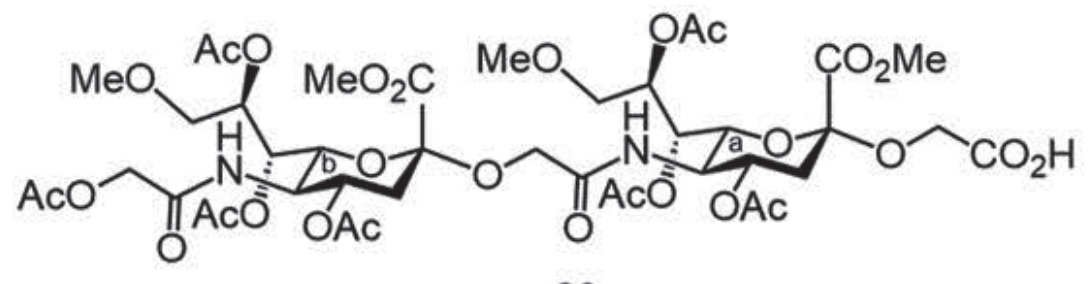

${ }^{13} \mathrm{C}$ NMR (125 MHz, $\mathrm{CDCl}_{3}$ ) 

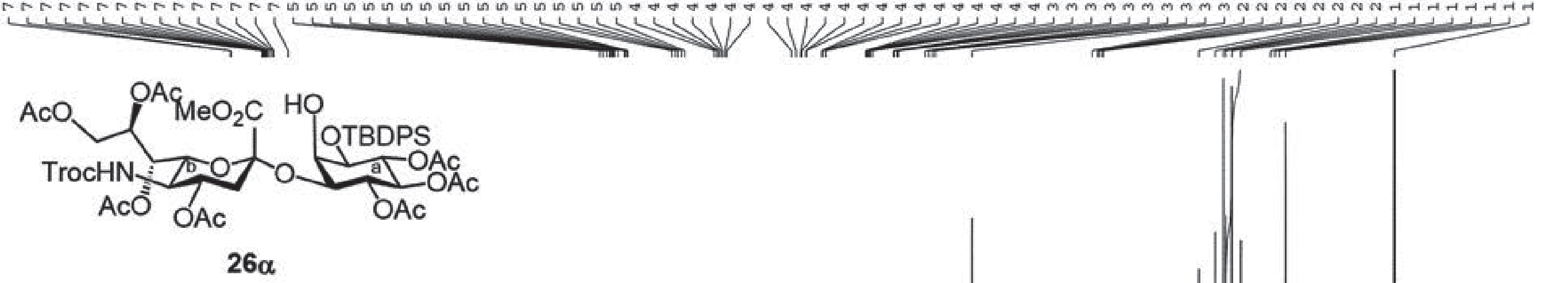

${ }^{1} \mathrm{H} \mathrm{NMR}\left(500 \mathrm{MHz}, \mathrm{CDCl}_{3}\right)$
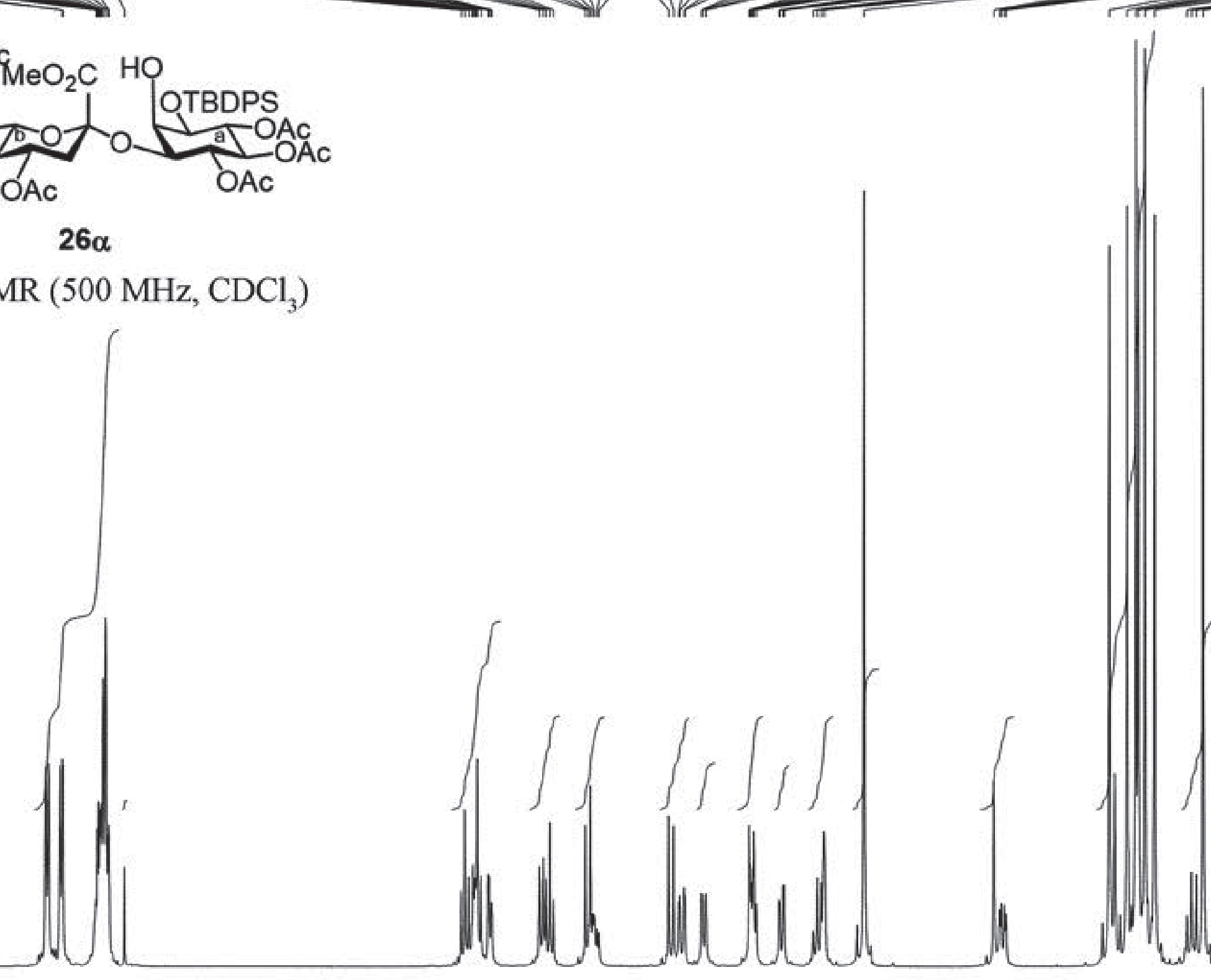

S44

8.5

8.0

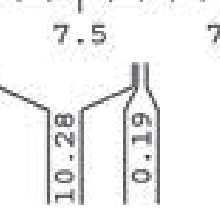

6.0

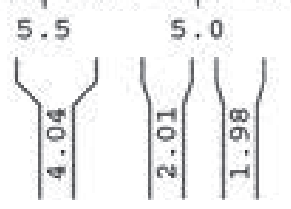

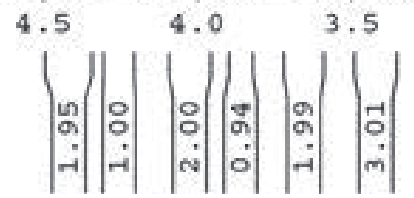

3.0
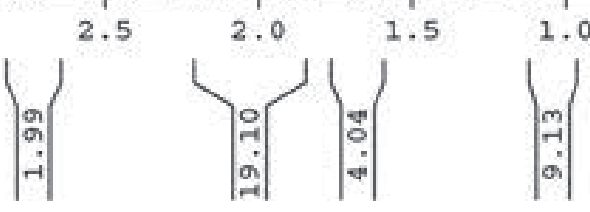

0.5

$0 \cdot 0$

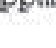


$\infty$ ம i 0 o 000 \% नतनतनतन त्नत 다다
๒ an 0

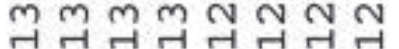

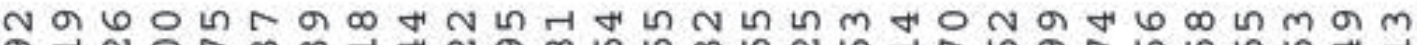

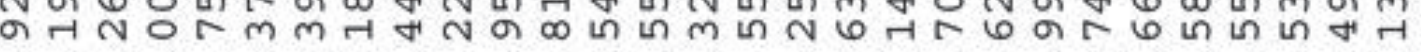
ब

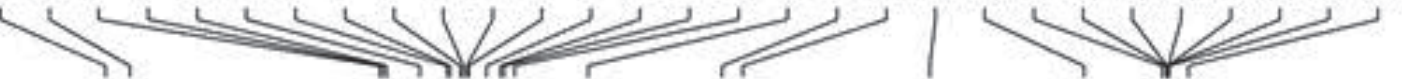

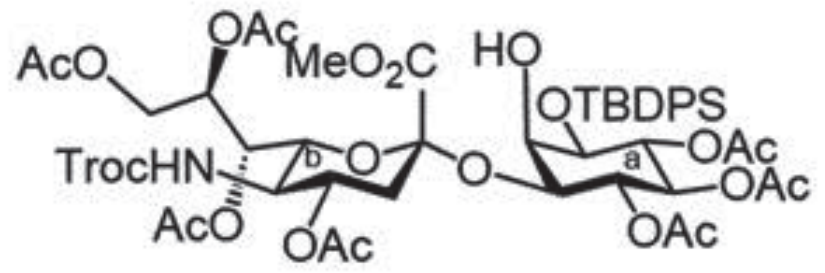

$26 \alpha$

${ }^{13} \mathrm{C} \mathrm{NMR}\left(125 \mathrm{MHz}, \mathrm{CDCl}_{3}\right.$ ) 


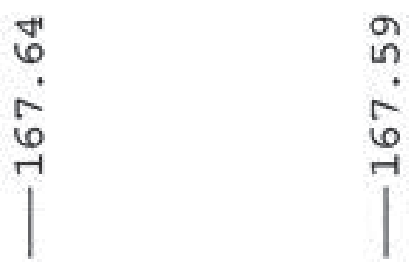

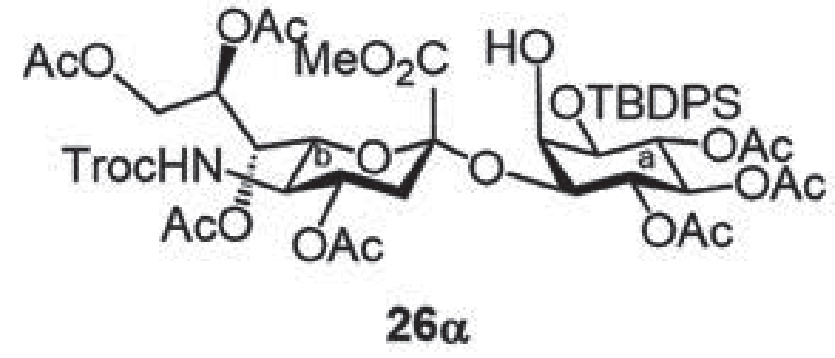

Gated $\left\{{ }^{1} \mathrm{H}\right\}{ }^{13} \mathrm{C}$ NMR $\left(125 \mathrm{MHz}, \mathrm{CDCl}_{3}\right)$

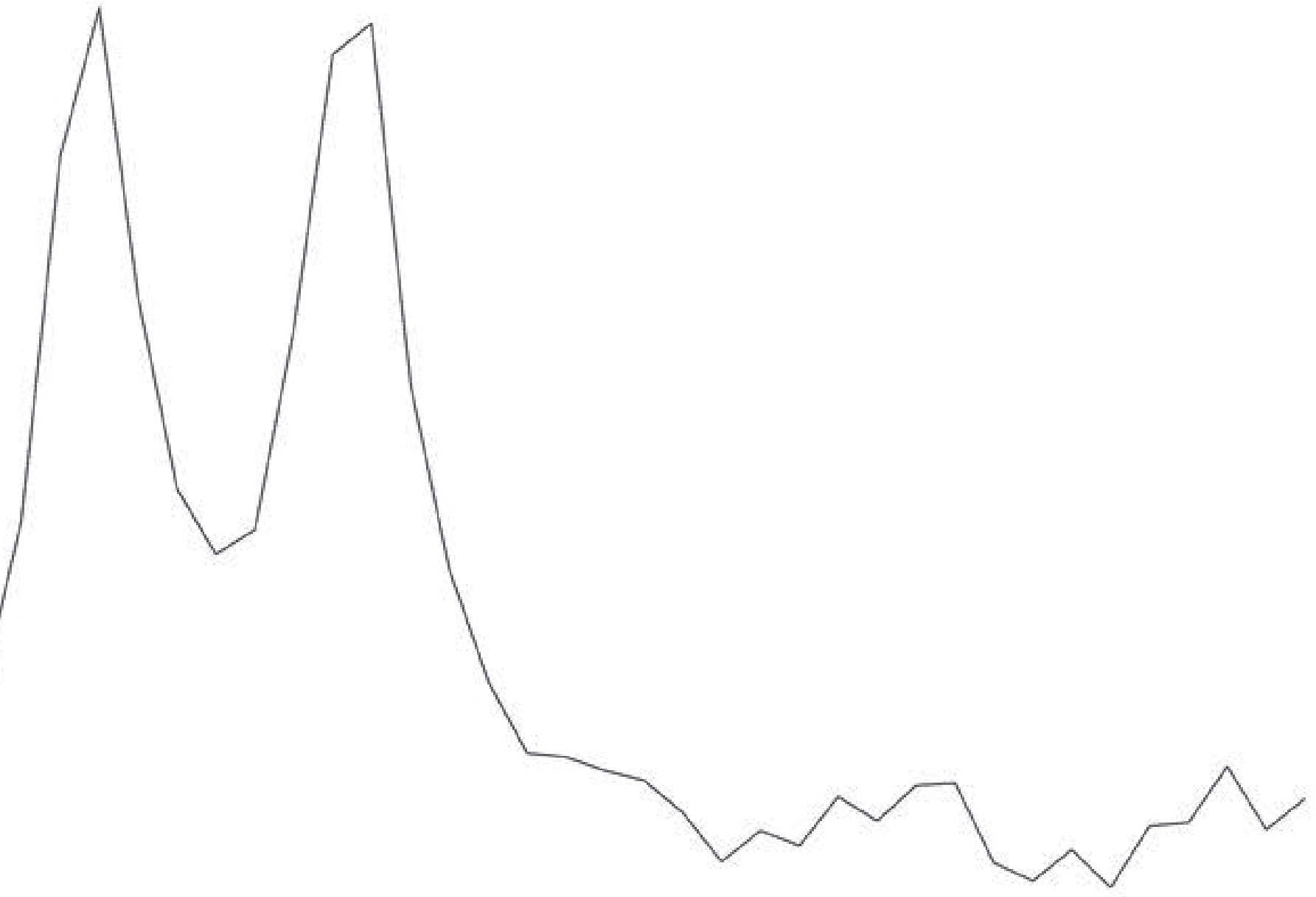




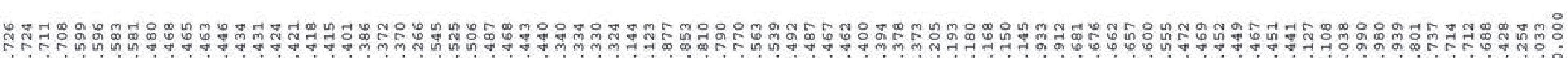

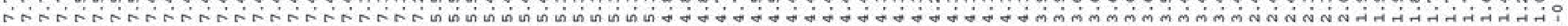

$\mathrm{HO}$

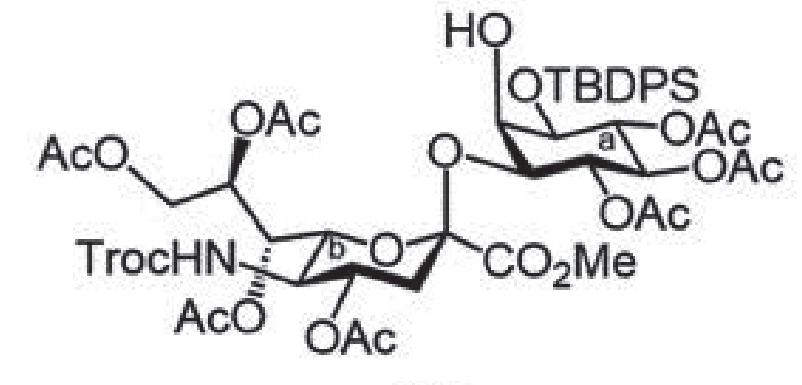

$26 \beta$

${ }^{1} \mathrm{H} \mathrm{NMR}\left(500\left(\mathrm{MHz}, \mathrm{CDCl}_{3}\right)\right.$

8.5
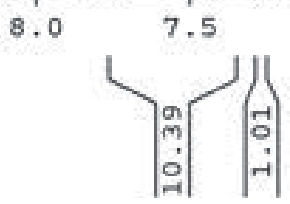

6.0
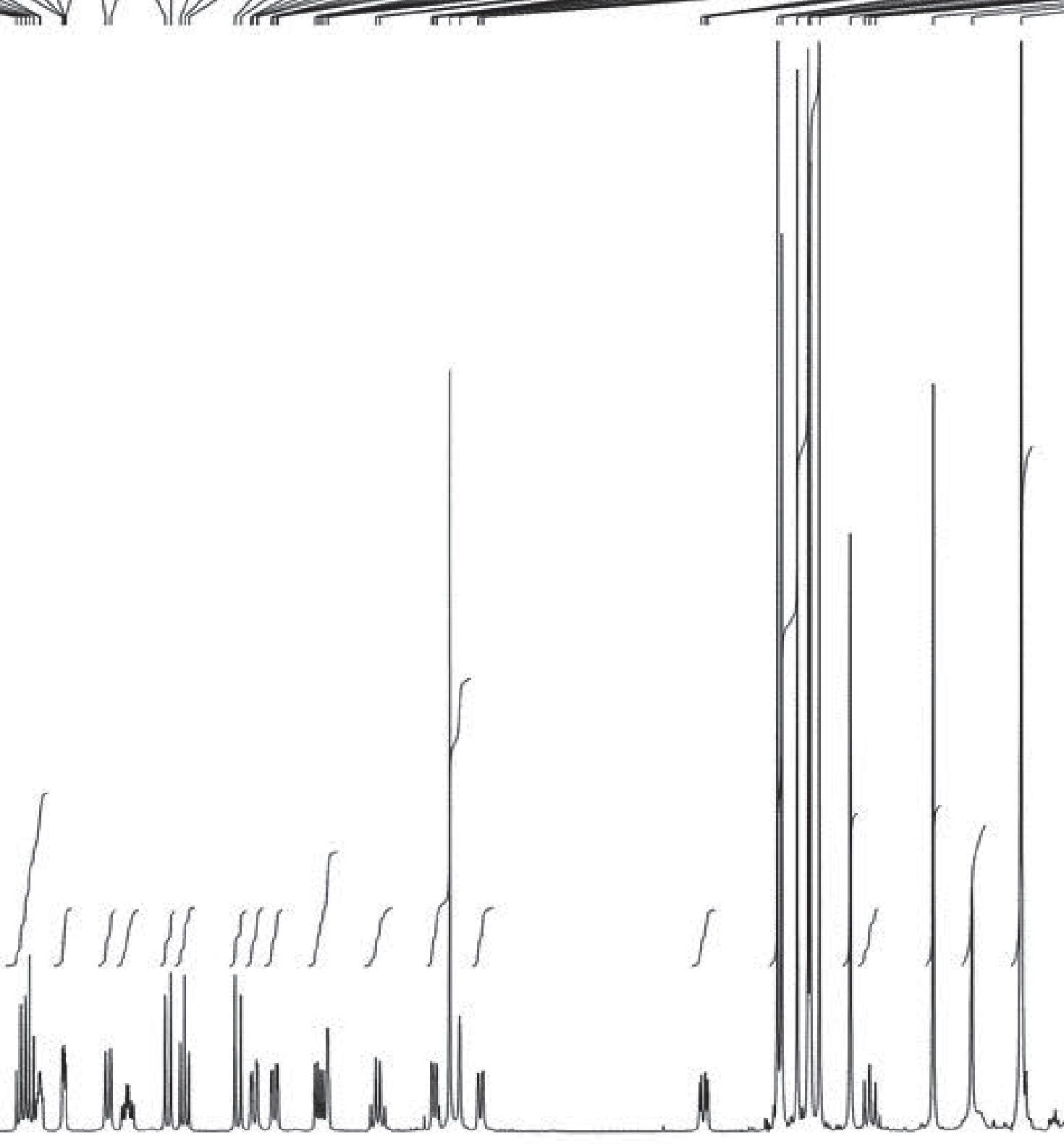

S47

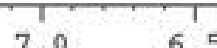

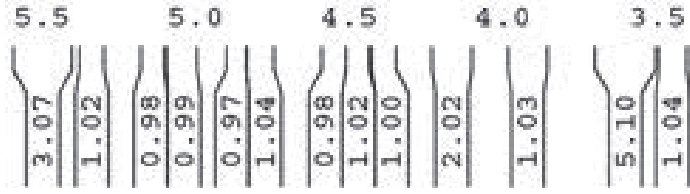

3.0

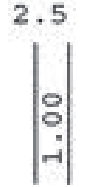

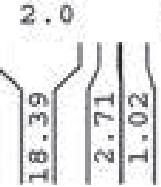

1.0

0.5

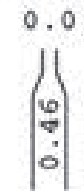




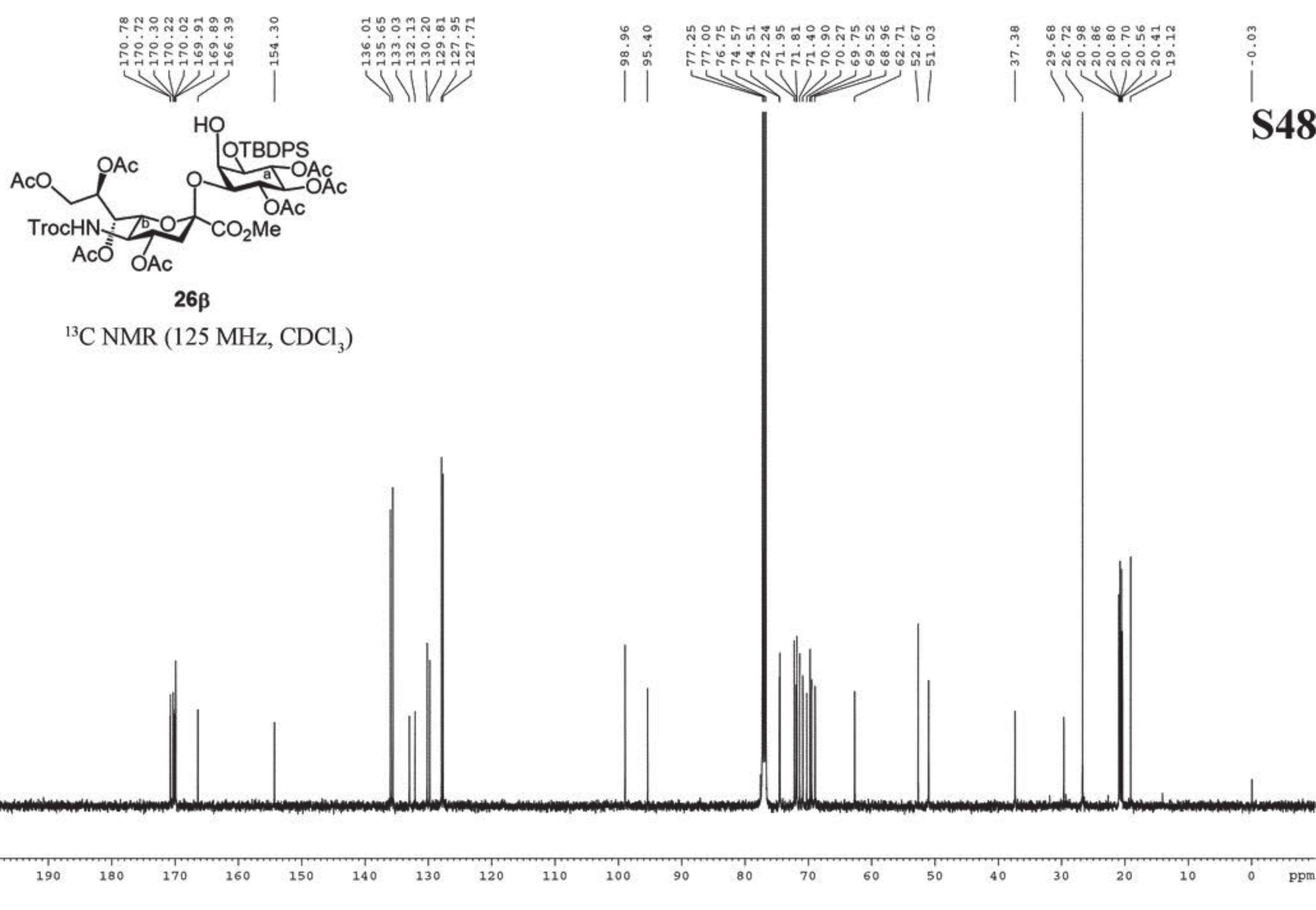




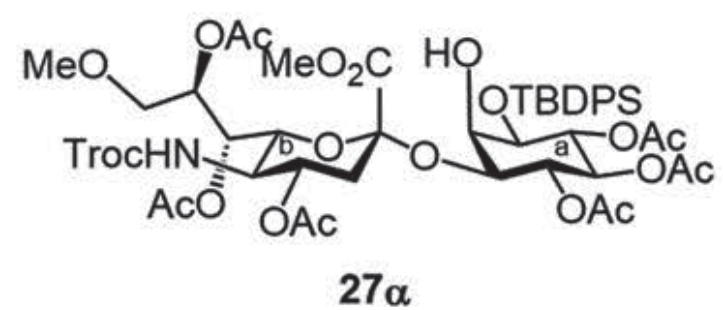

${ }^{1} \mathrm{H}$ NMR ( $500 \mathrm{MHz}, \mathrm{CDCl}_{3}$ )

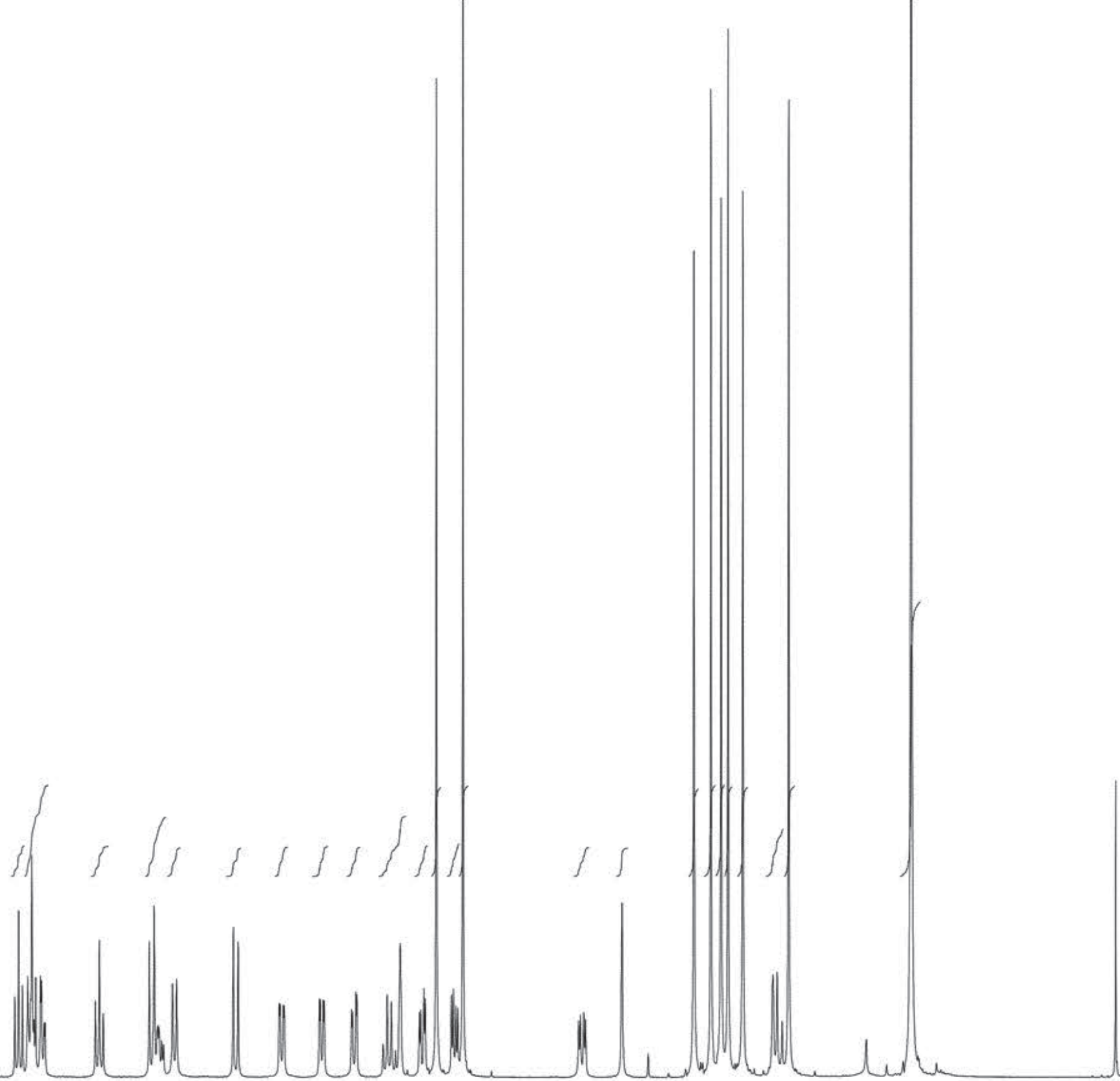




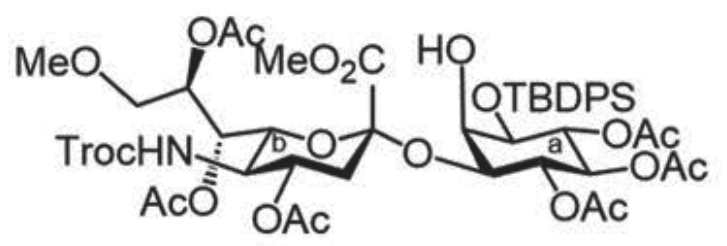

$27 \alpha$

${ }^{13} \mathrm{C}$ NMR (125 MHz, $\mathrm{CDCl}_{3}$ )
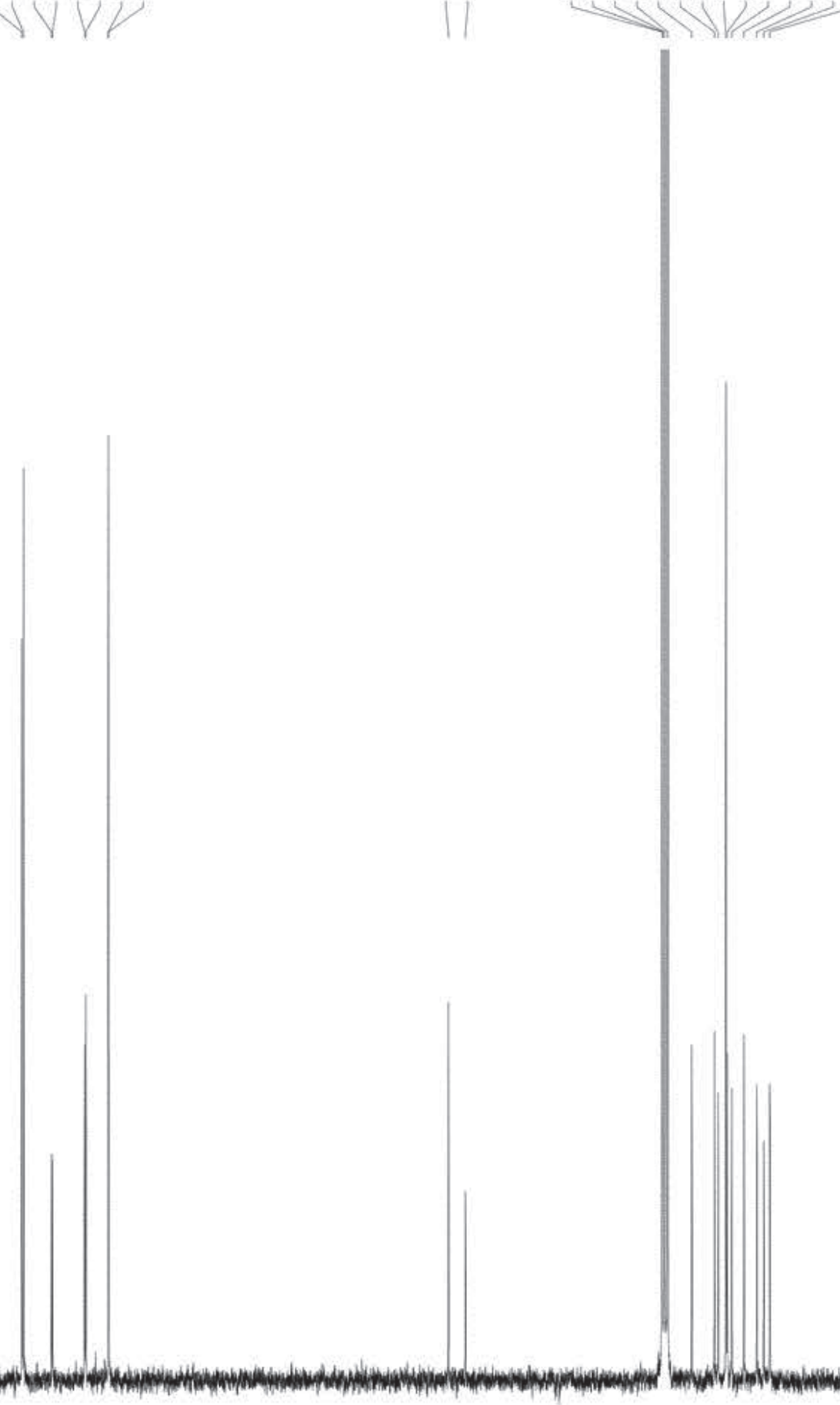

S50 


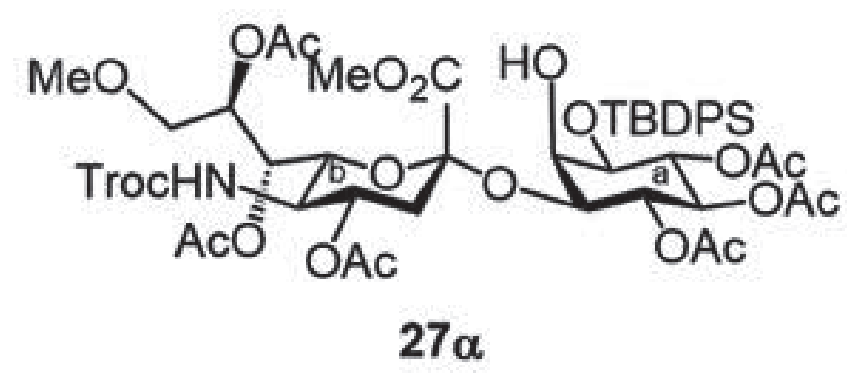

Gated $\left\{{ }^{1} \mathrm{H}\right\}{ }^{13} \mathrm{C}$ NMR $\left(125 \mathrm{MHz}, \mathrm{CDCl}_{3}\right)$

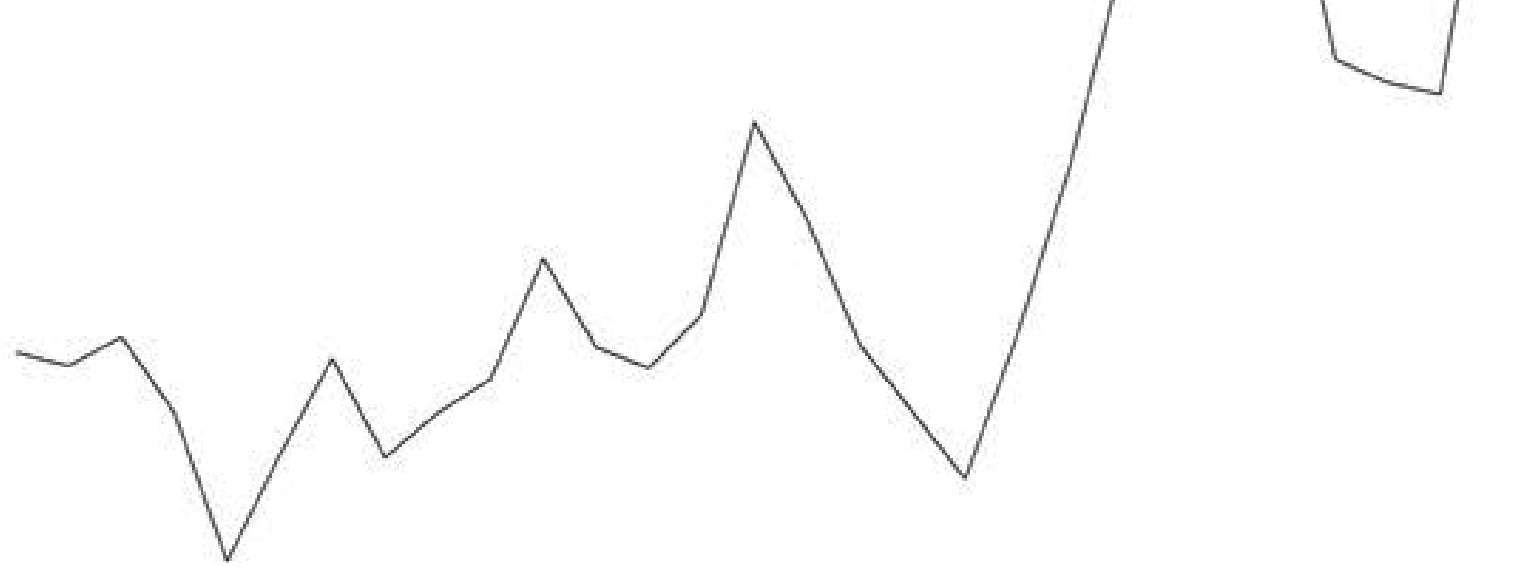

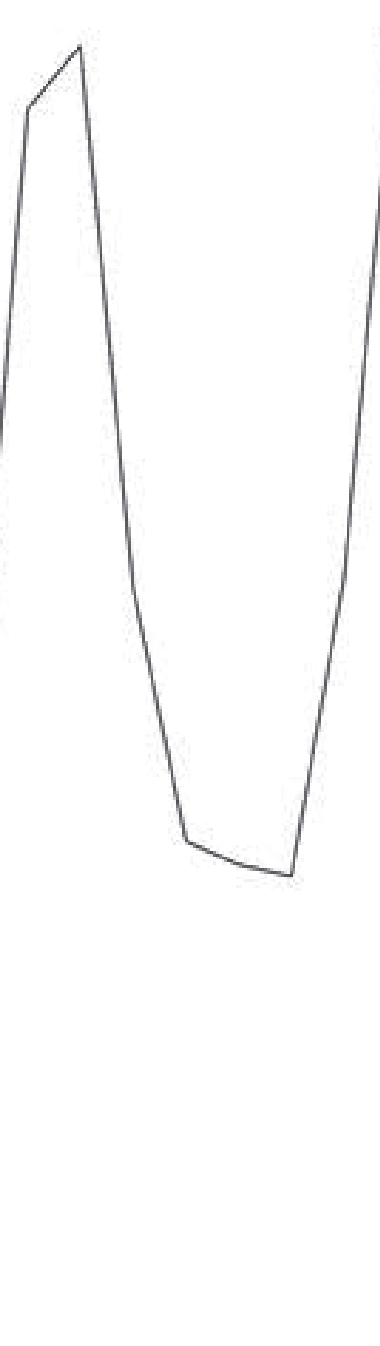

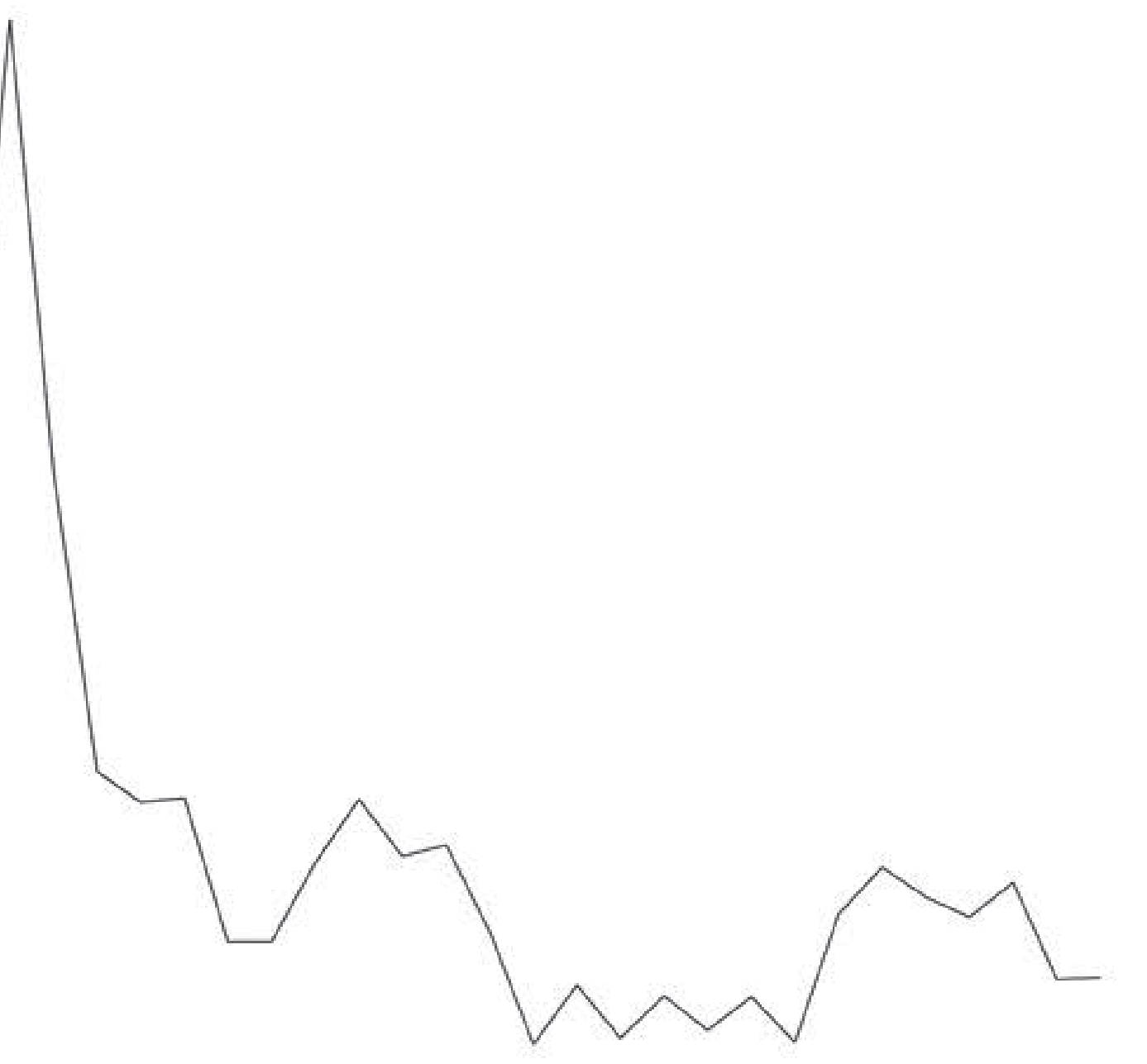




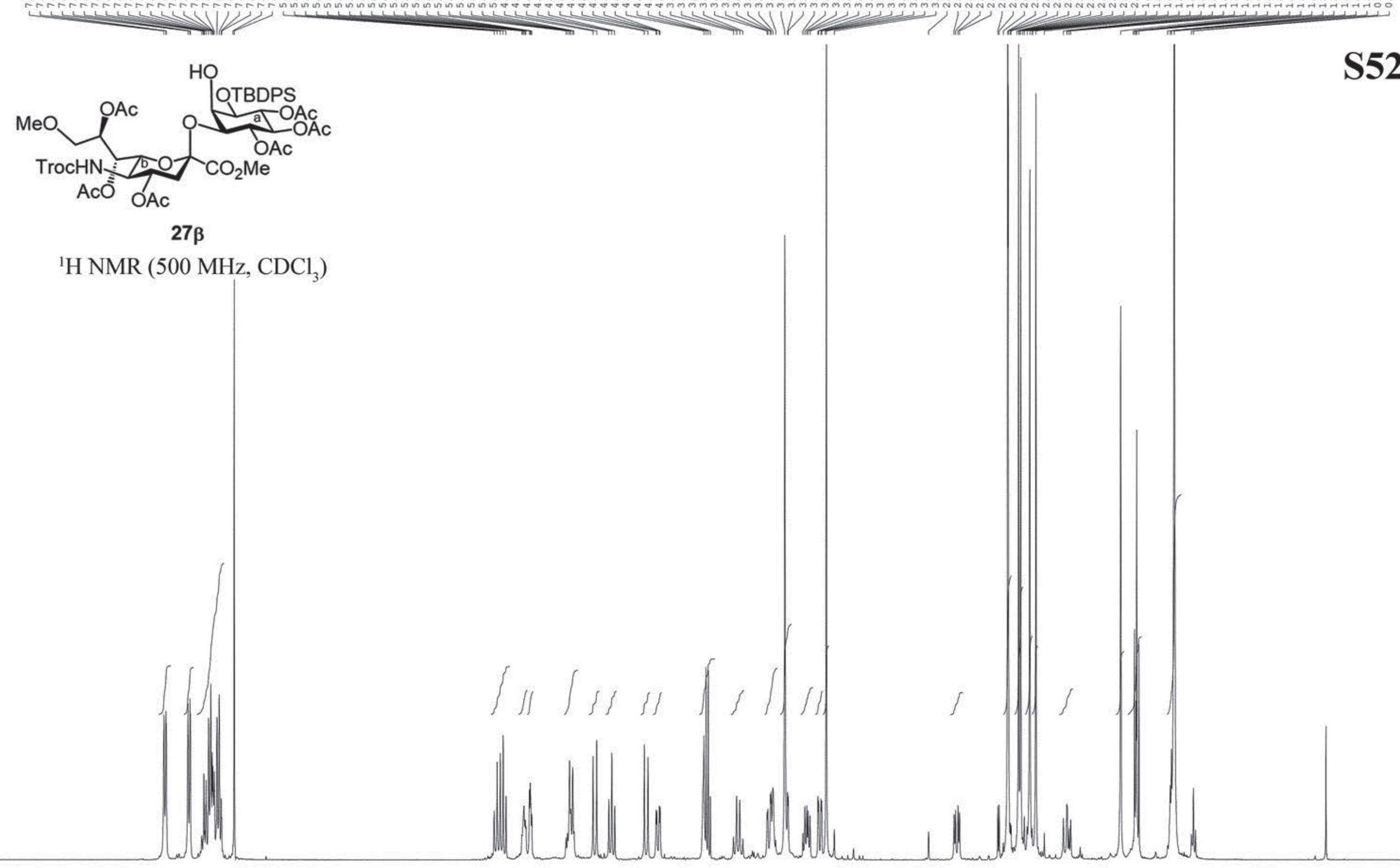



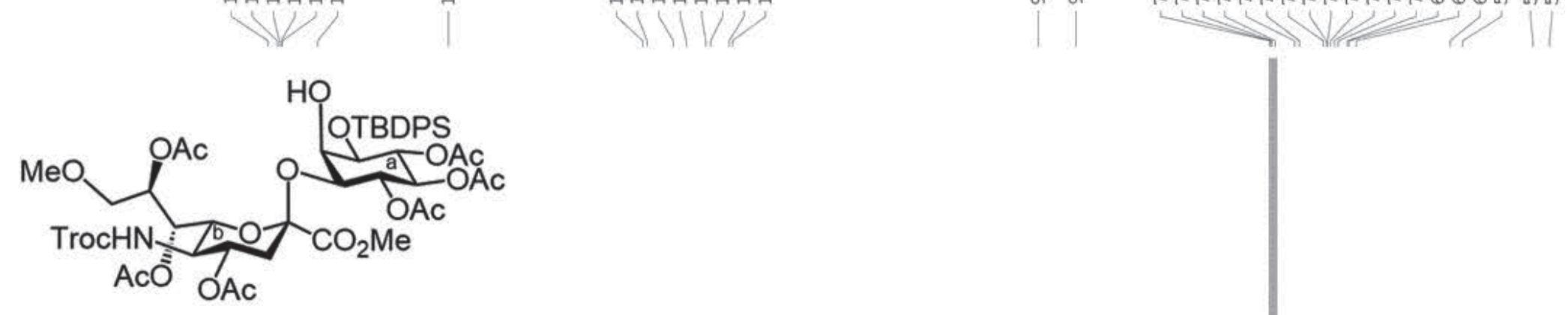

ติ

S53

$27 \beta$

${ }^{13} \mathrm{C}$ NMR $\left(125 \mathrm{MHz}, \mathrm{CDCl}_{3}\right)$

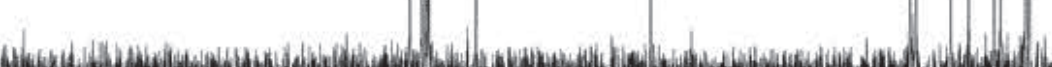

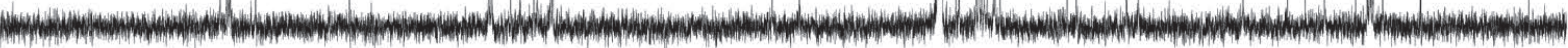




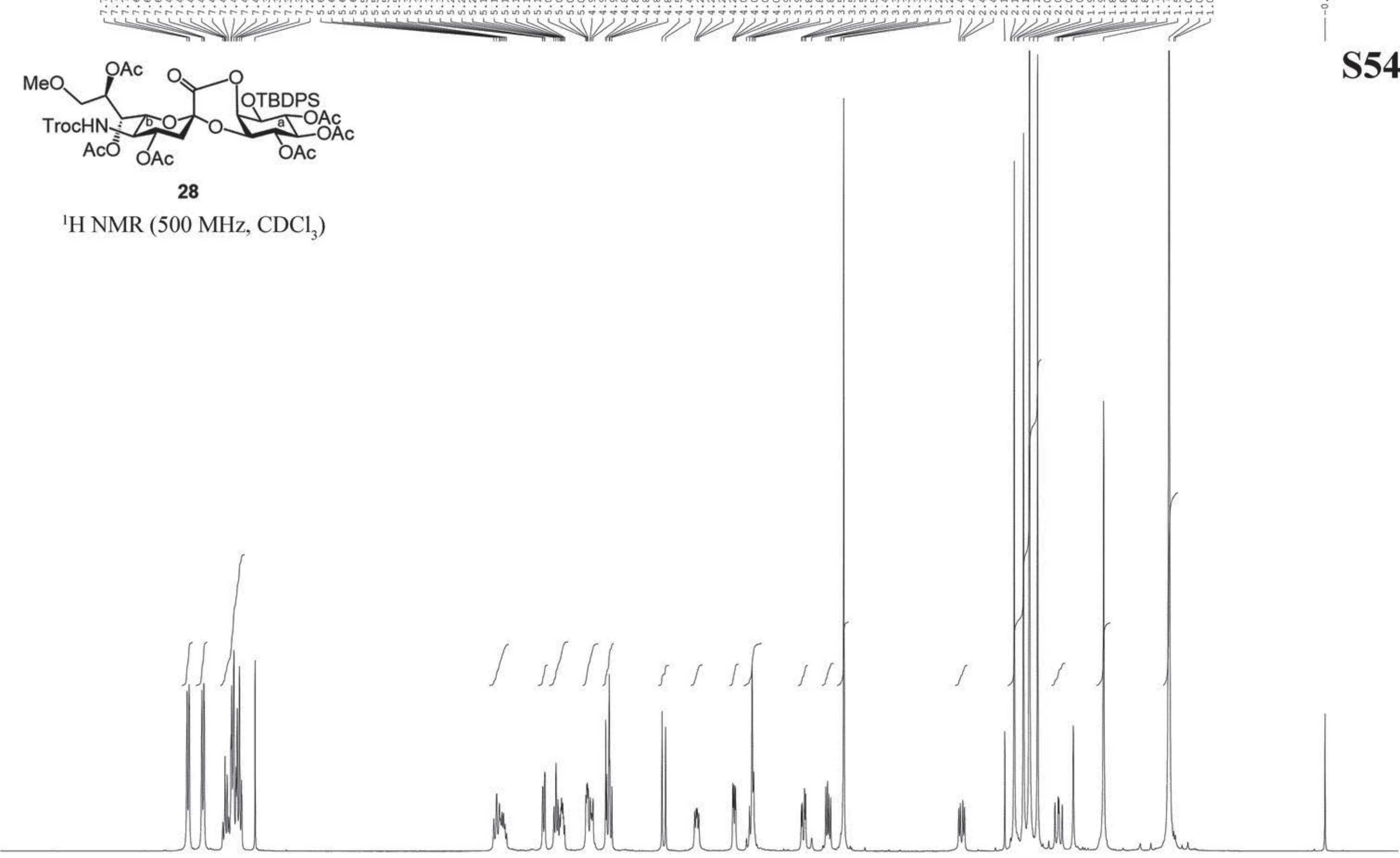




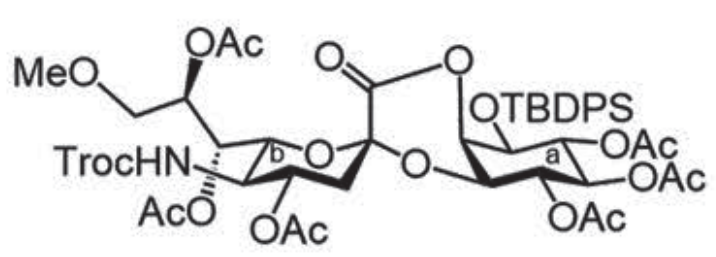

28

${ }^{13} \mathrm{C} \mathrm{NMR}\left(125 \mathrm{MHz}, \mathrm{CDCl}_{3}\right)$
मून

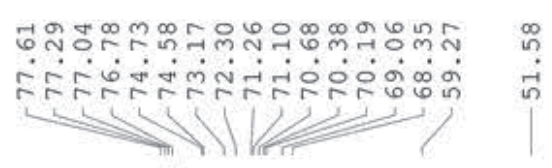

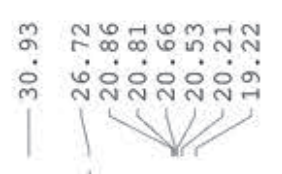

S55 


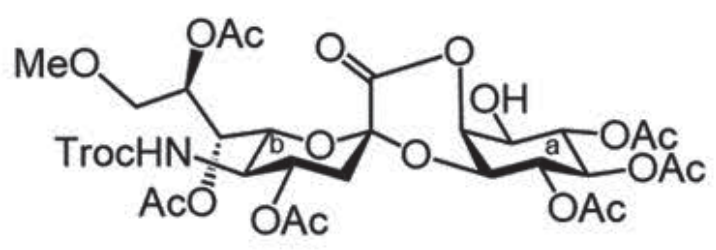

29

${ }^{1} \mathrm{H} \mathrm{NMR}\left(500 \mathrm{MHz}, \mathrm{CDCl}_{3}\right)$
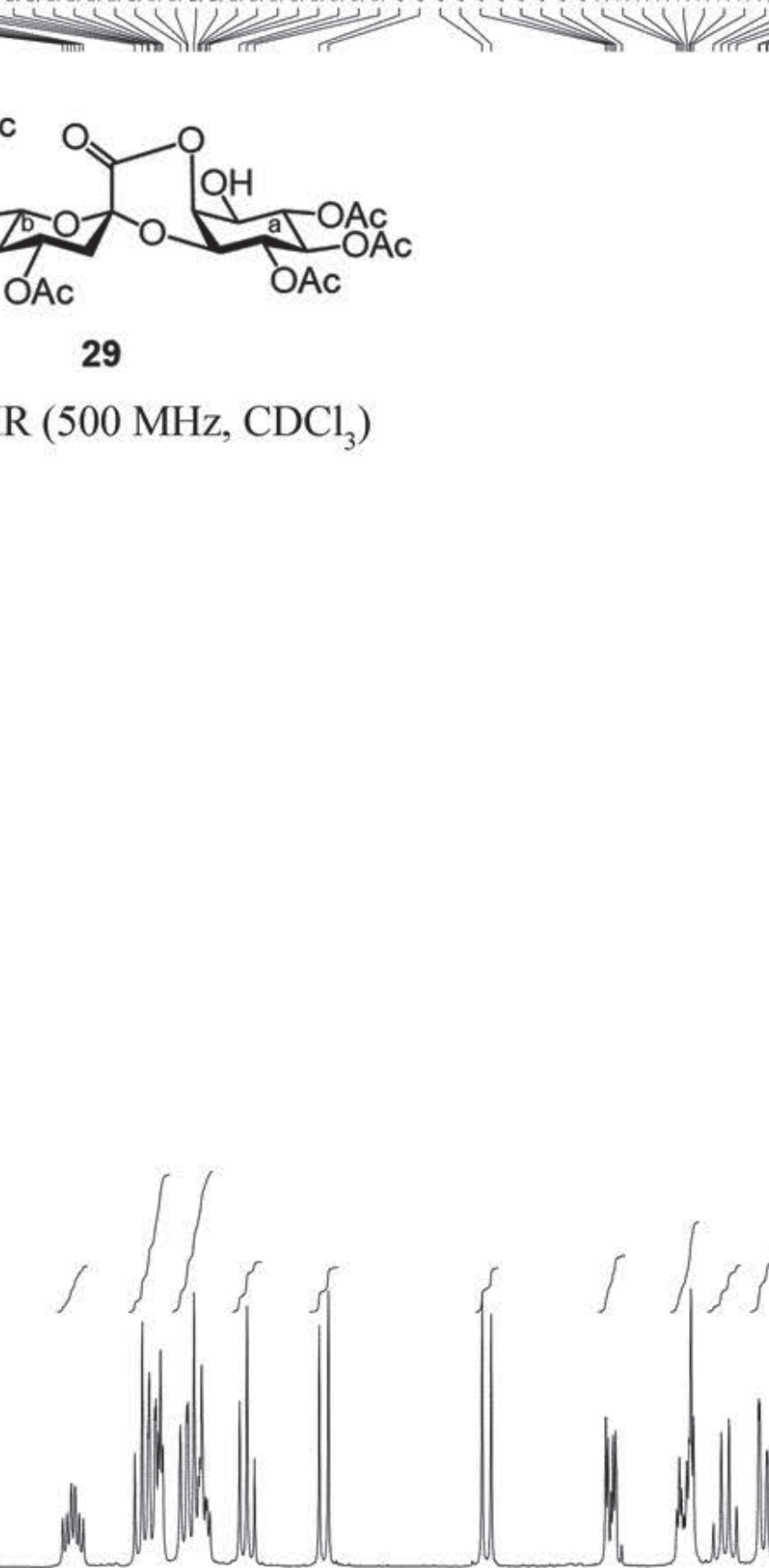

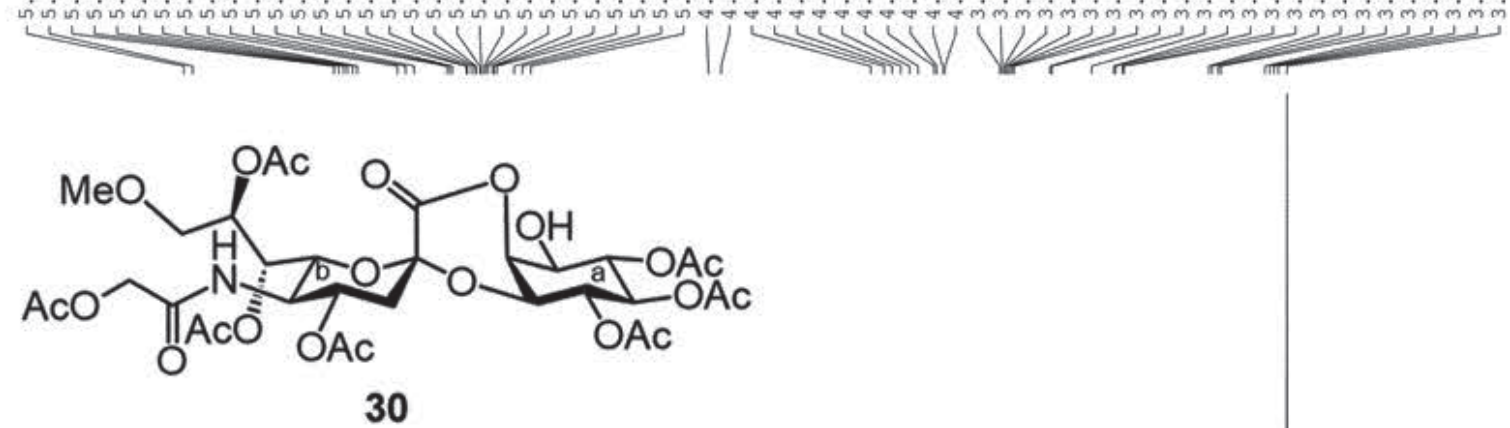

${ }^{1} \mathrm{H} \mathrm{NMR}\left(500 \mathrm{MHz}, \mathrm{CDCl}_{3}\right)$

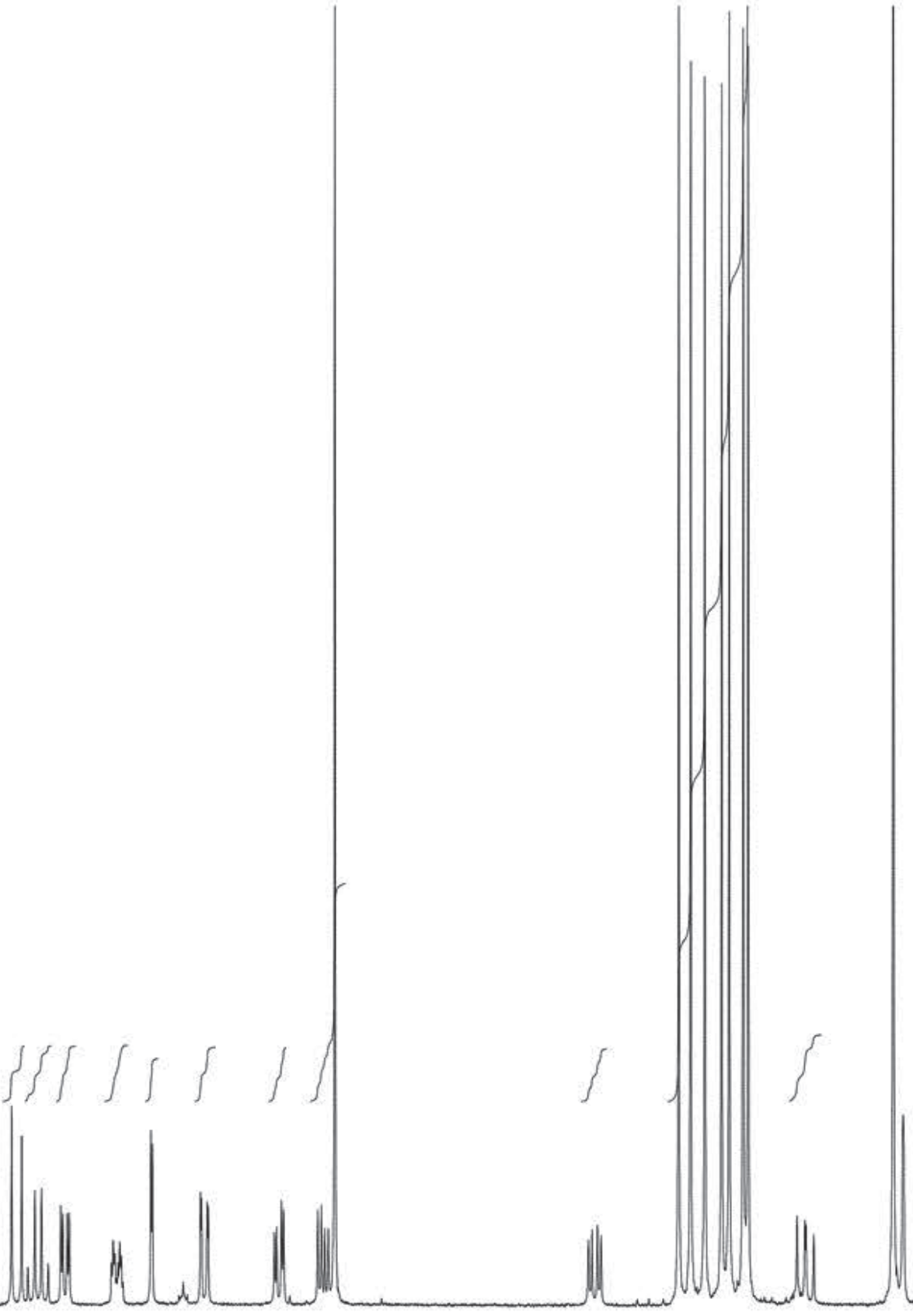

S58

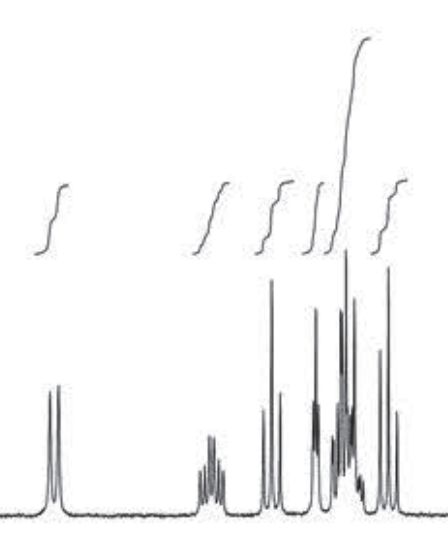

3.0

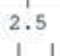

1.5

1.0

0.5

0.0 


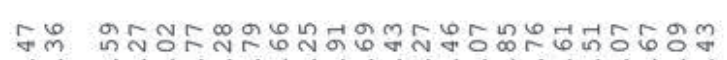

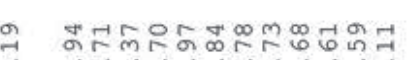
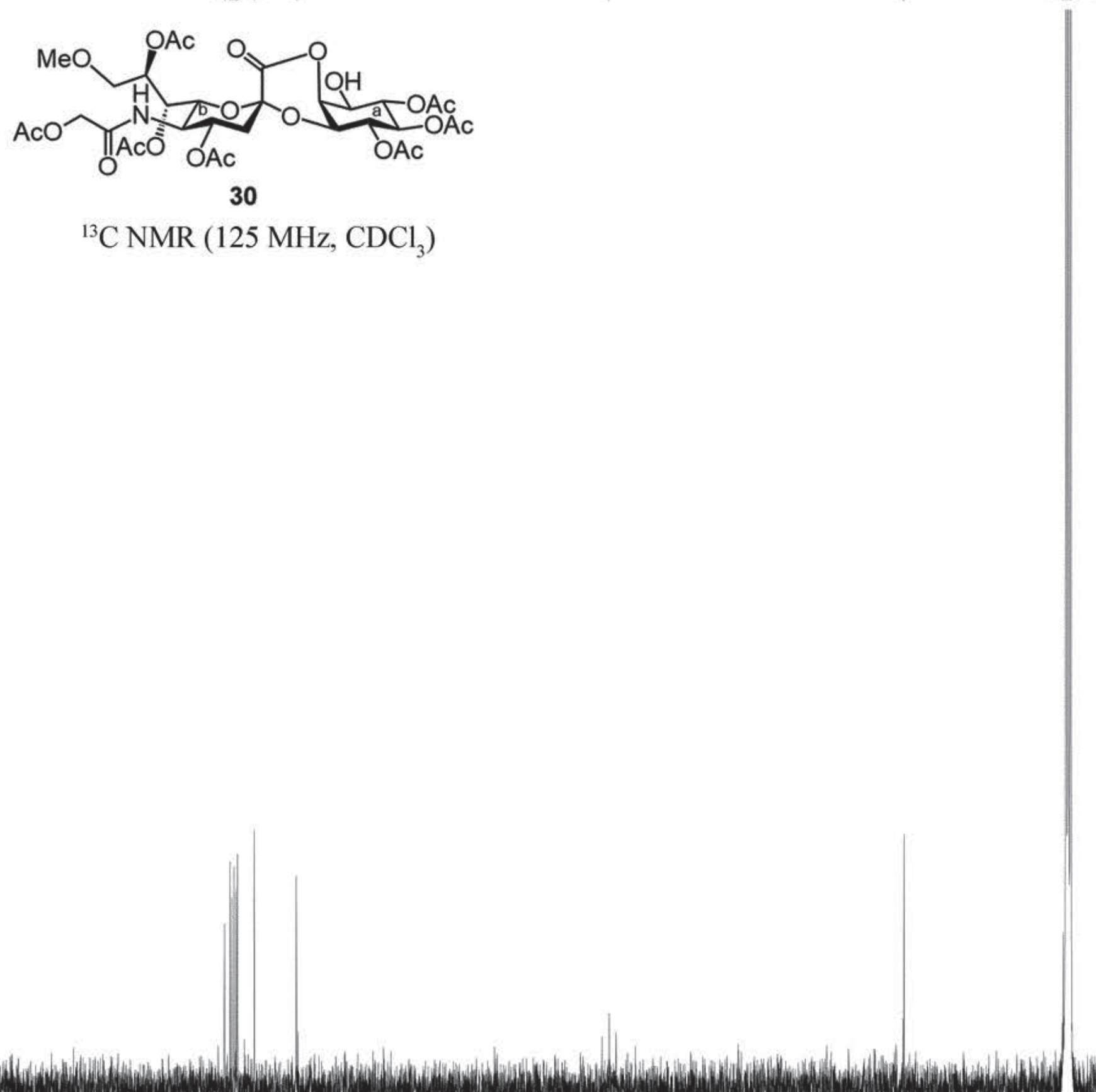

S59

${ }^{13} \mathrm{C}$ NMR (125 MHz, $\left.\mathrm{CDCl}_{3}\right)$

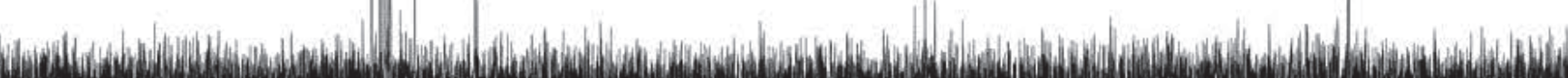

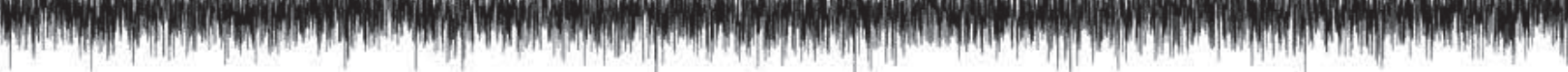

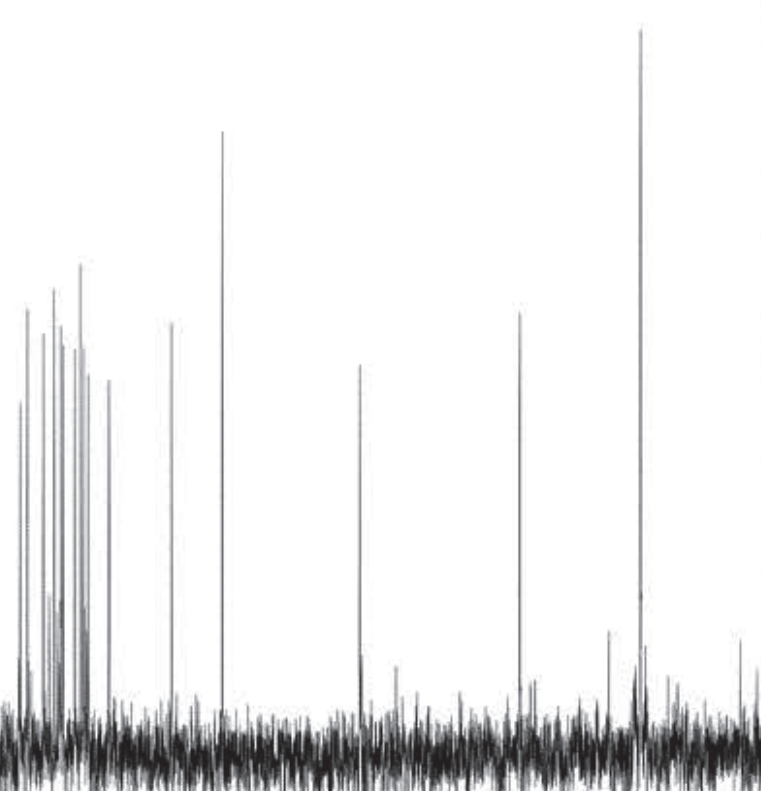



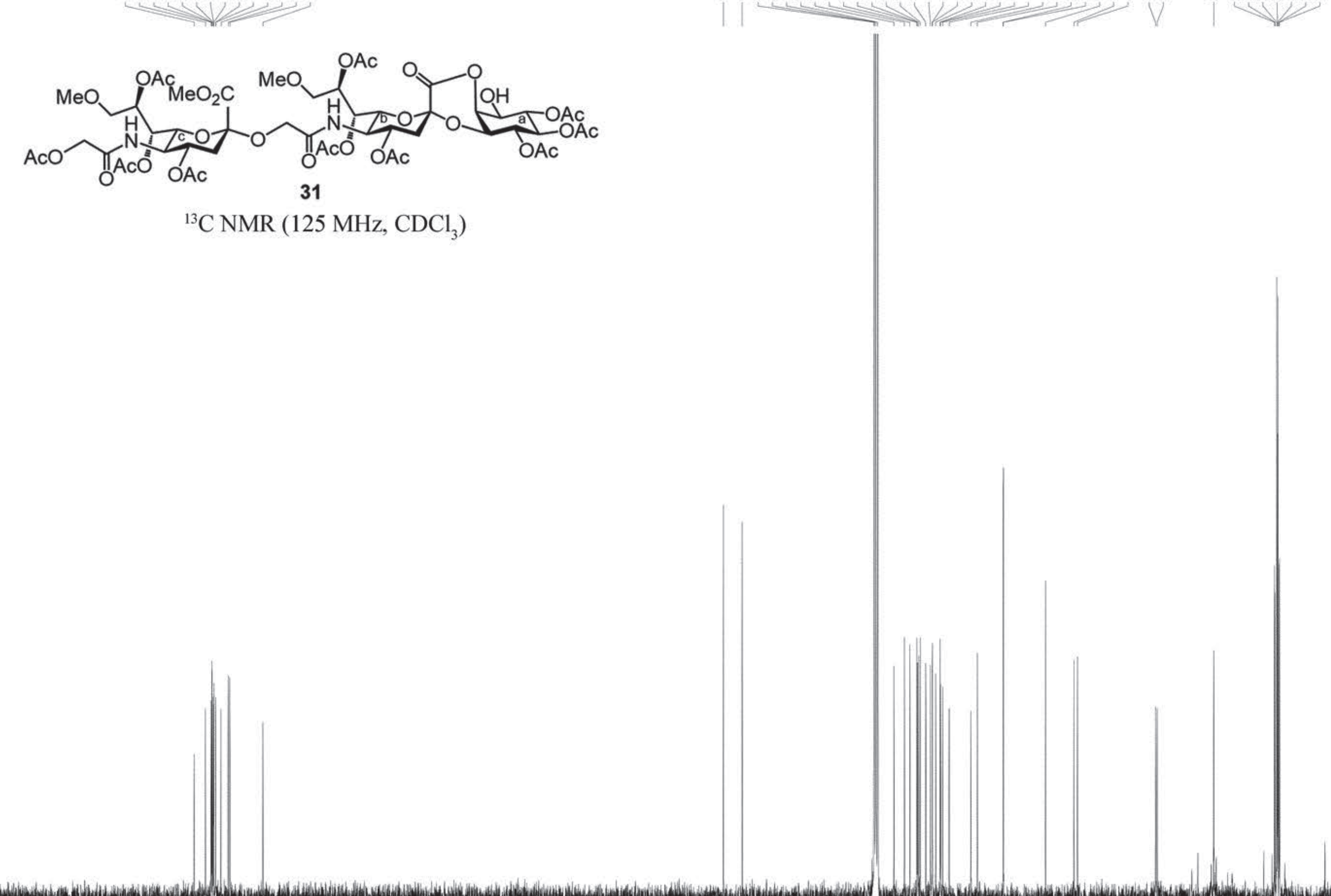

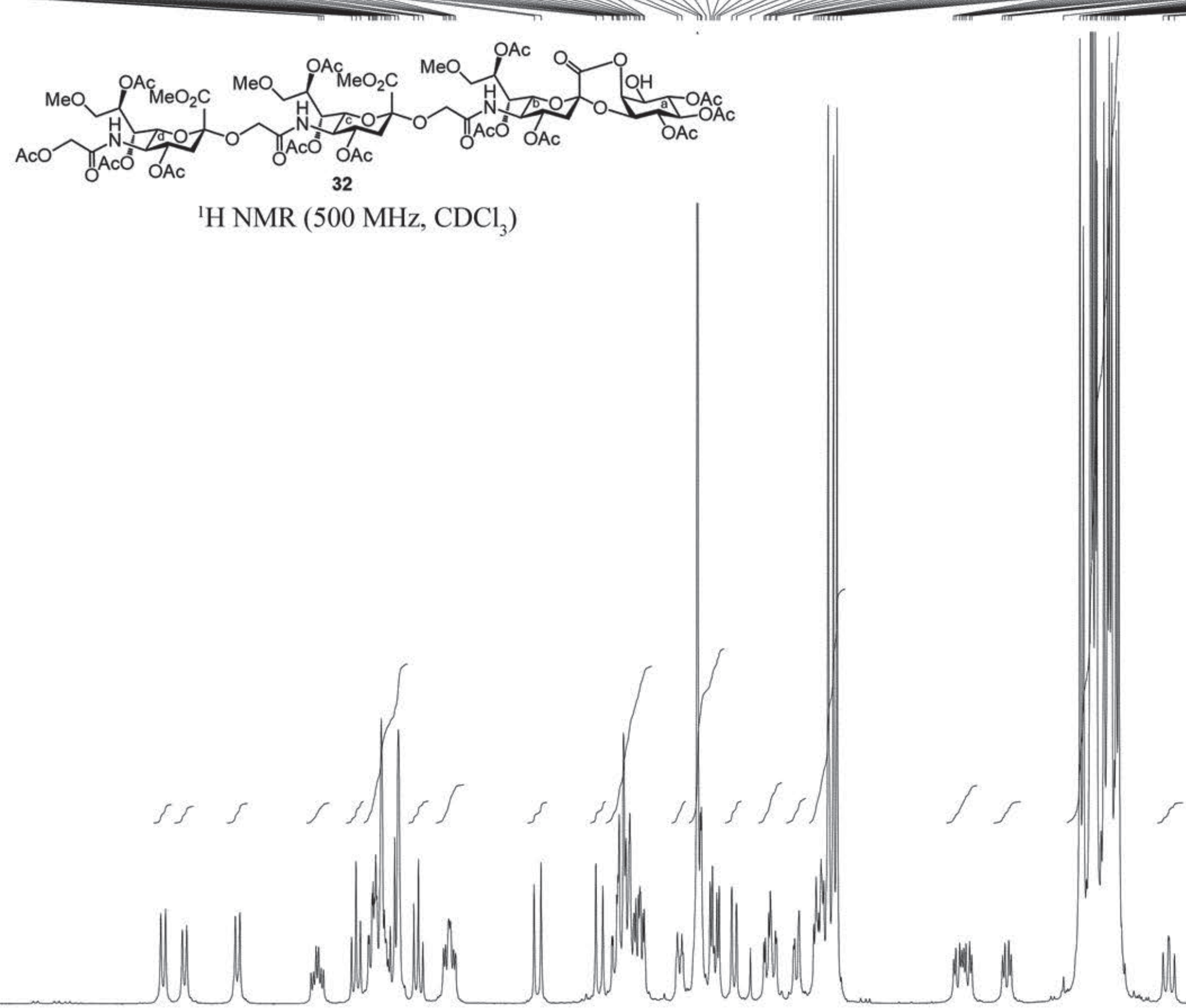

S62 


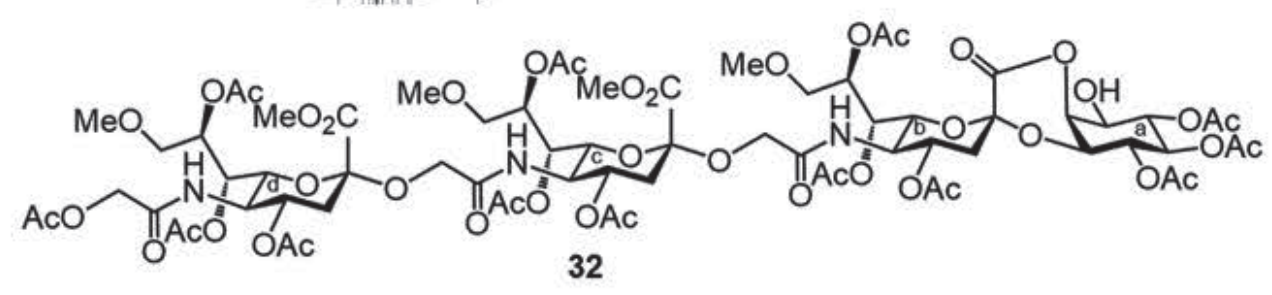

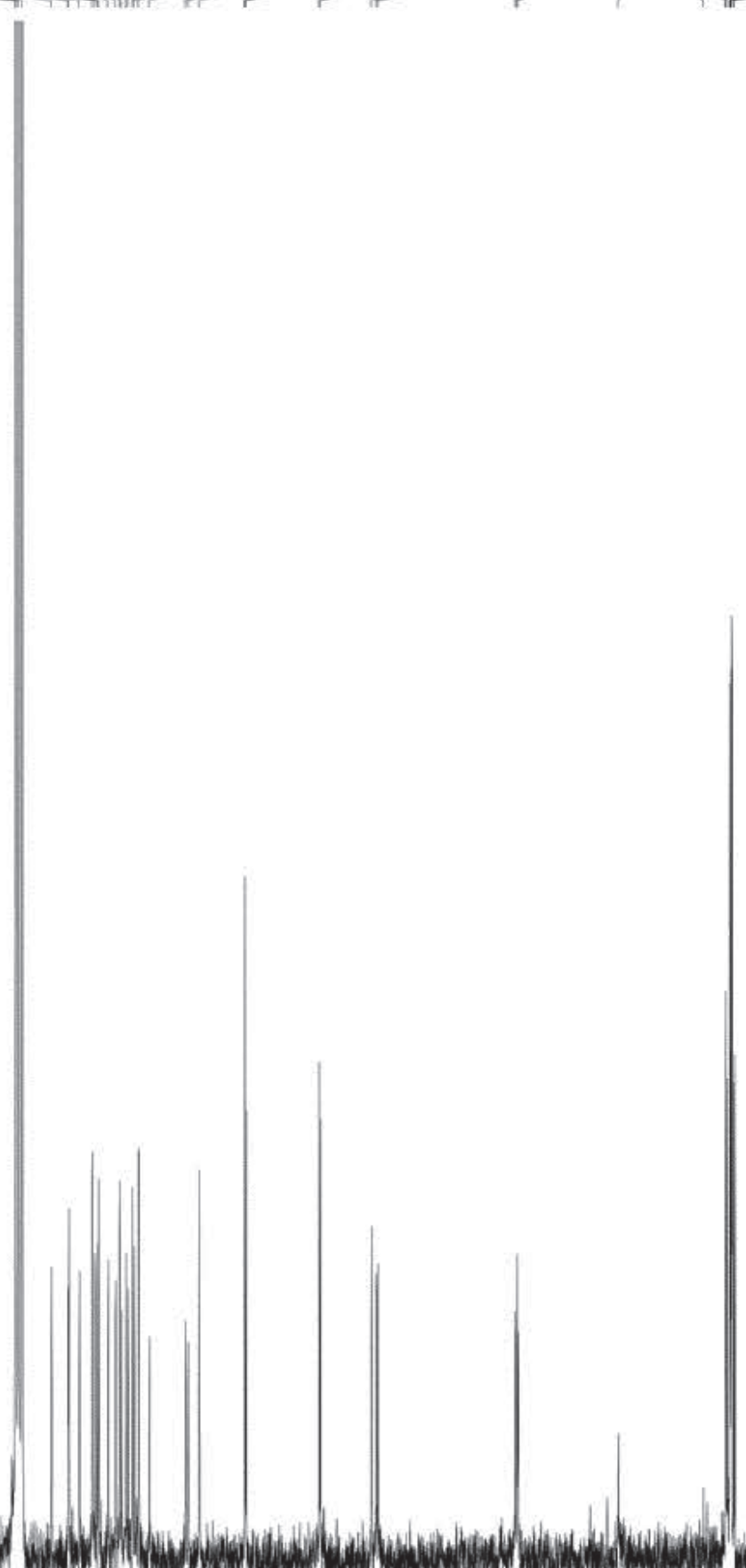

S63

${ }^{13} \mathrm{C}$ NMR (125 MHz, $\mathrm{CDCl}_{3}$ ) 


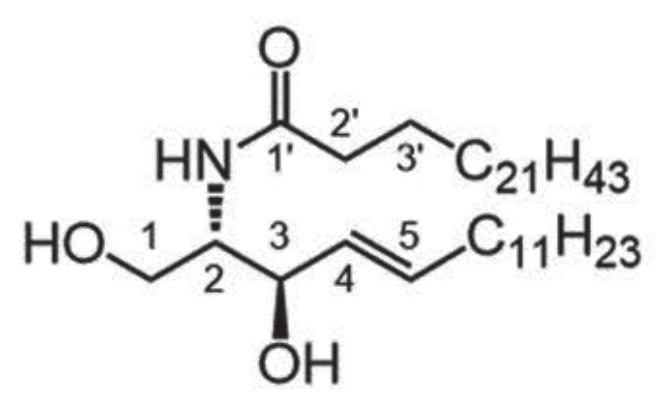

S1

${ }^{1} \mathrm{H} \mathrm{NMR}\left(500 \mathrm{MHz}, \mathrm{CDCl}_{3}\right.$ ) 


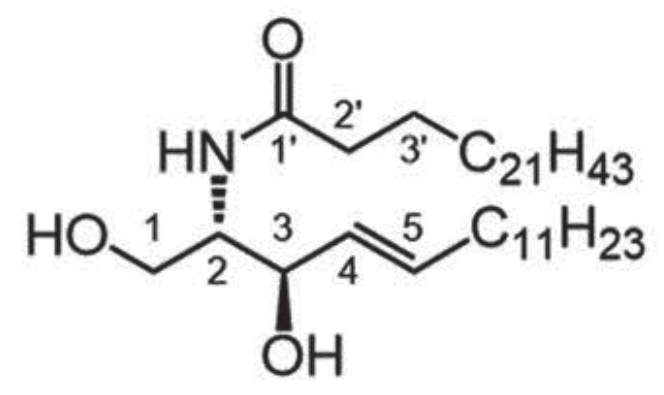

\section{S1}

${ }^{13} \mathrm{C}$ NMR (125 MHz, $\mathrm{CDCl}_{3}$ )

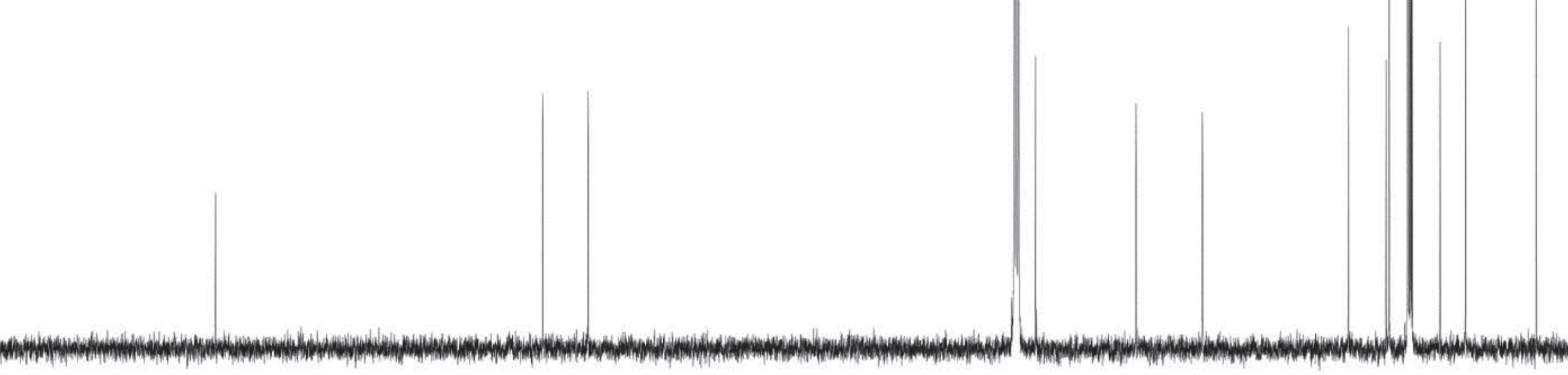

S65 


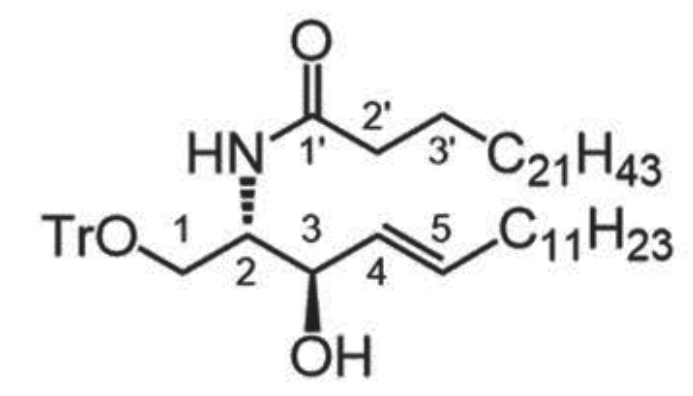

S2

${ }^{1} \mathrm{H} \mathrm{NMR}\left(500 \mathrm{MHz}, \mathrm{CDCl}_{3}\right)$

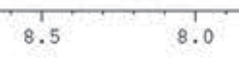
$7.0 \quad 6.5$
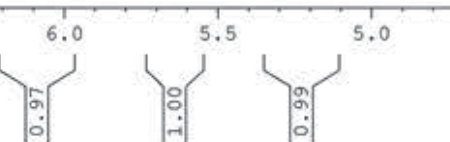


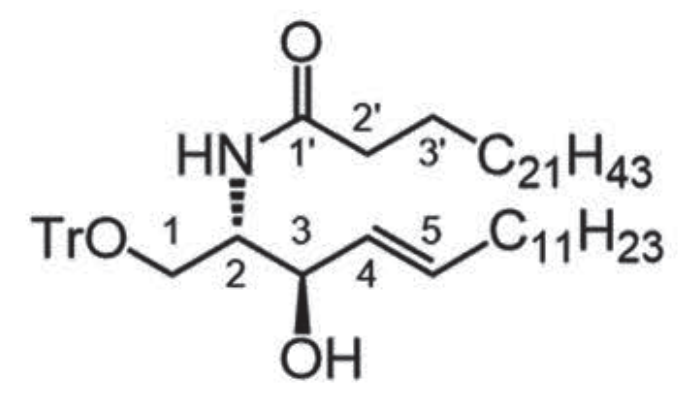

\section{S2}

${ }^{13} \mathrm{C} \mathrm{NMR}\left(125 \mathrm{MHz}, \mathrm{CDCl}_{3}\right)$ 


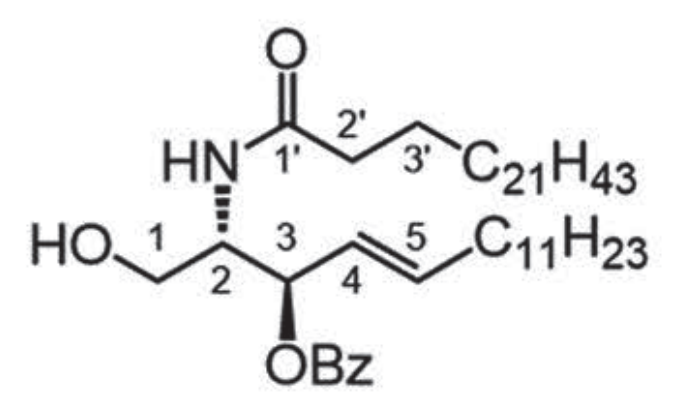

S68

\section{4}

${ }^{1} \mathrm{H} \mathrm{NMR}\left(500 \mathrm{MHz}, \mathrm{CDCl}_{3}\right)$

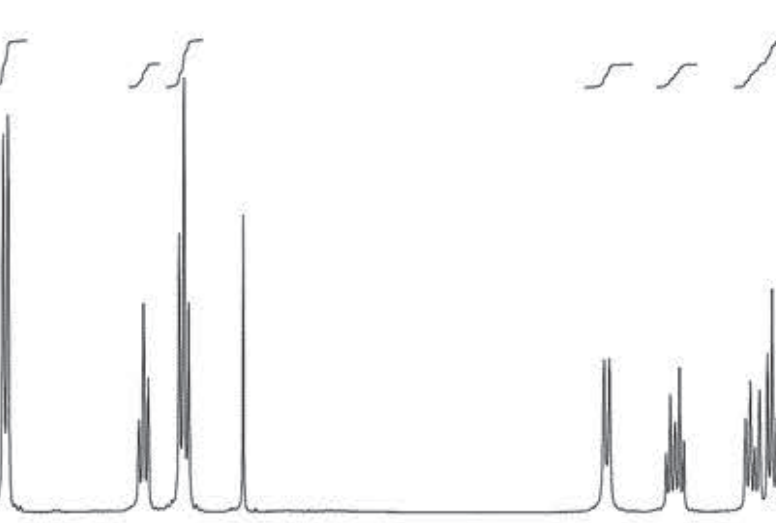




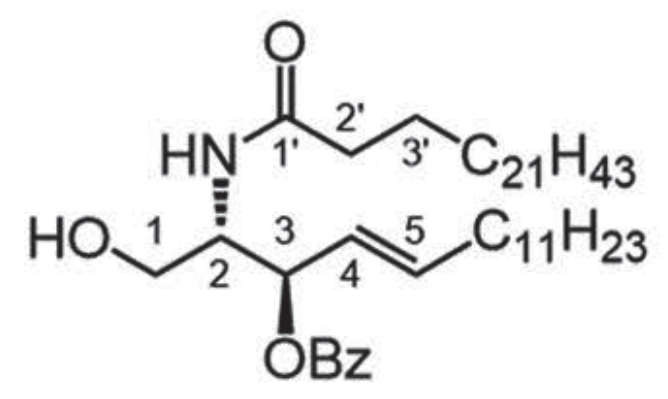

34

${ }^{13} \mathrm{C}$ NMR (125 MHz, $\left.\mathrm{CDCl}_{3}\right)$ 


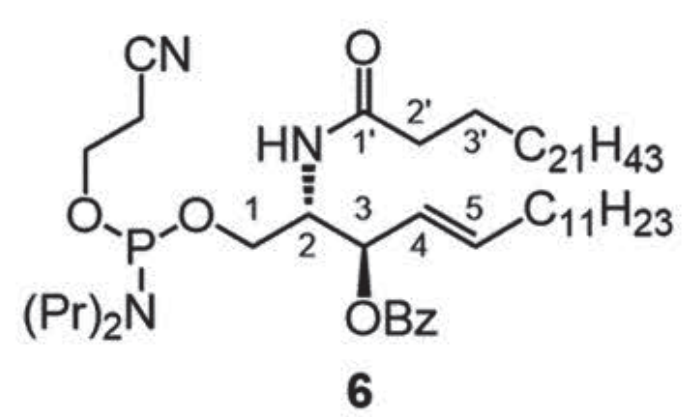

${ }^{1} \mathrm{H}$ NMR (500 MHz, $\mathrm{CDCl}_{3}$ )

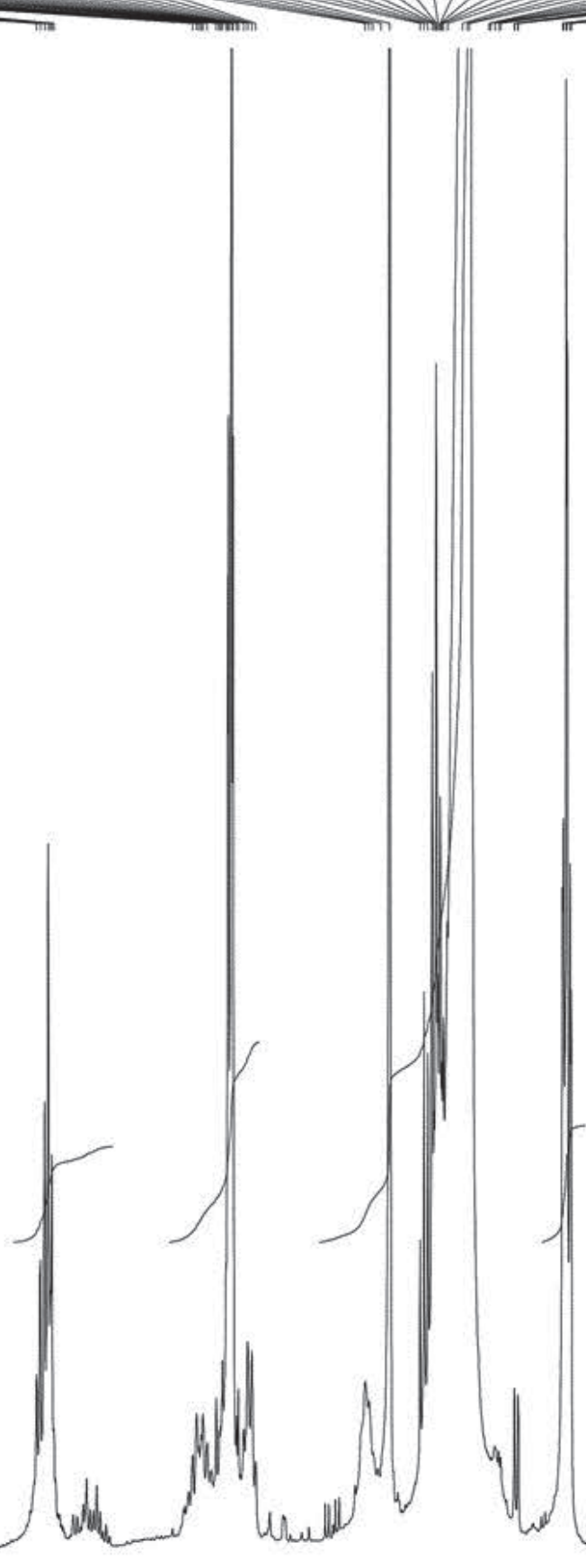




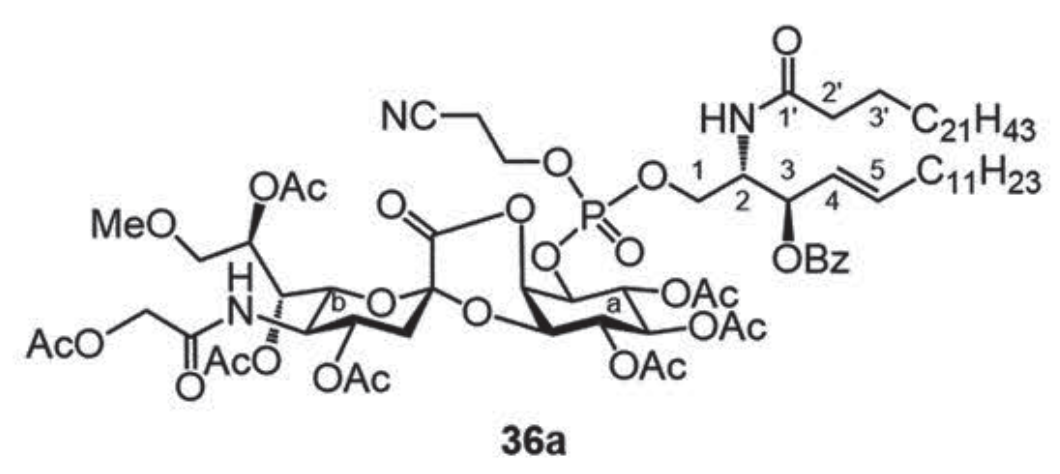

${ }^{1} \mathrm{H} \mathrm{NMR}\left(500 \mathrm{MHz}, \mathrm{CD}_{3} \mathrm{CN}\right)$
S71 


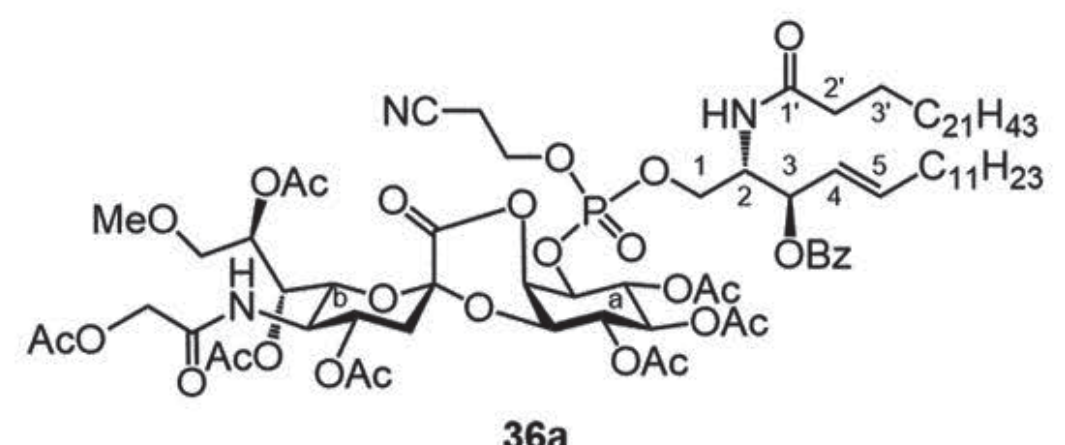

$36 a$

${ }^{13} \mathrm{C} \mathrm{NMR}\left(125 \mathrm{MHz}, \mathrm{CDCl}_{3}\right)$

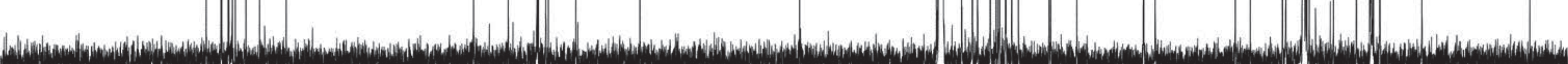

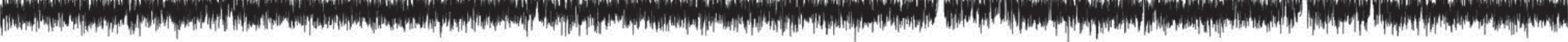

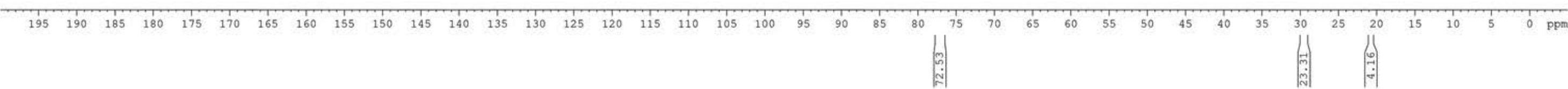




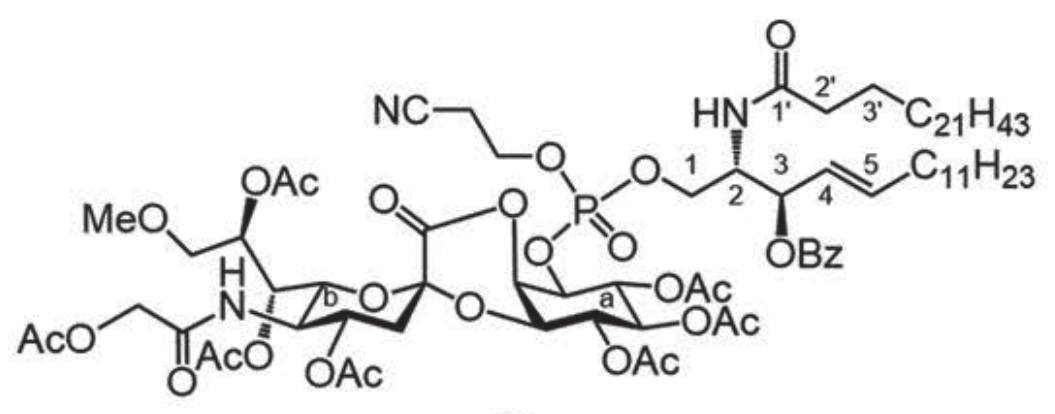

36 a

${ }^{31} \mathrm{P} \mathrm{NMR}\left(202 \mathrm{MHz}, \mathrm{CDCl}_{3}\right)$ 


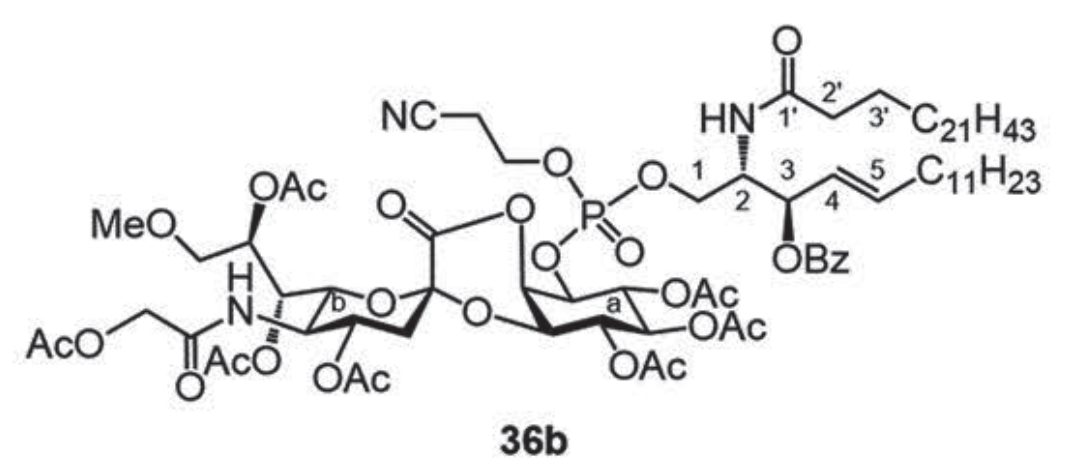

${ }^{1} \mathrm{H} \operatorname{NMR}\left(500 \mathrm{MHz}, \mathrm{CDCl}_{3}\right.$ ) 


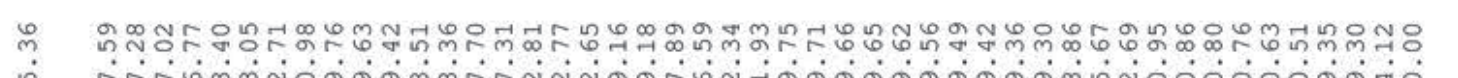

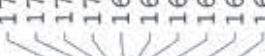

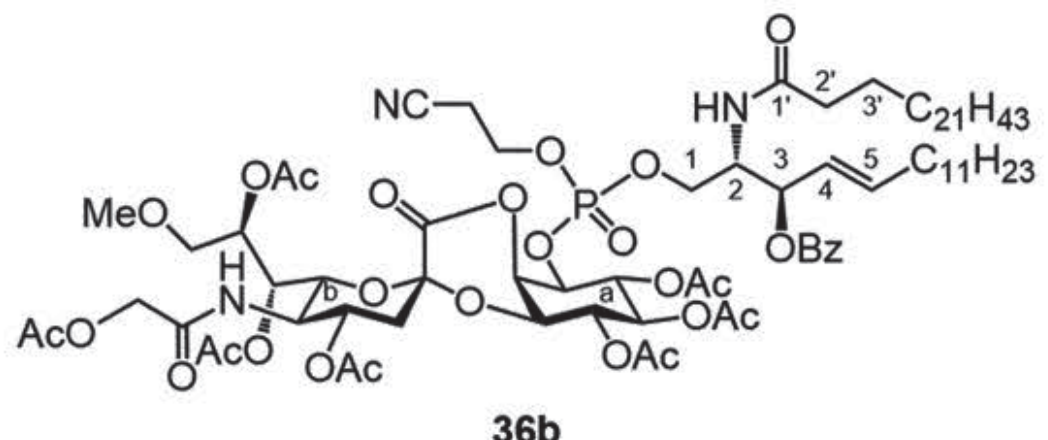

${ }^{13} \mathrm{C} \mathrm{NMR}\left(125 \mathrm{MHz}, \mathrm{CDCl}_{3}\right)$

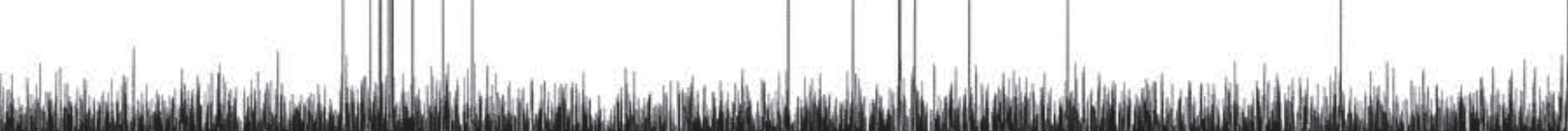

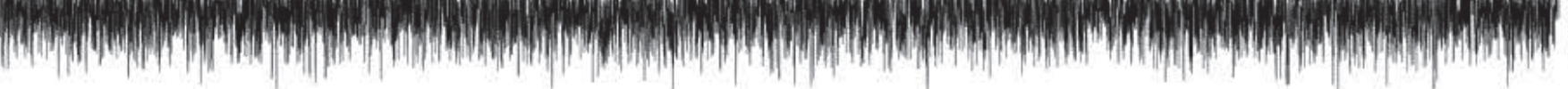

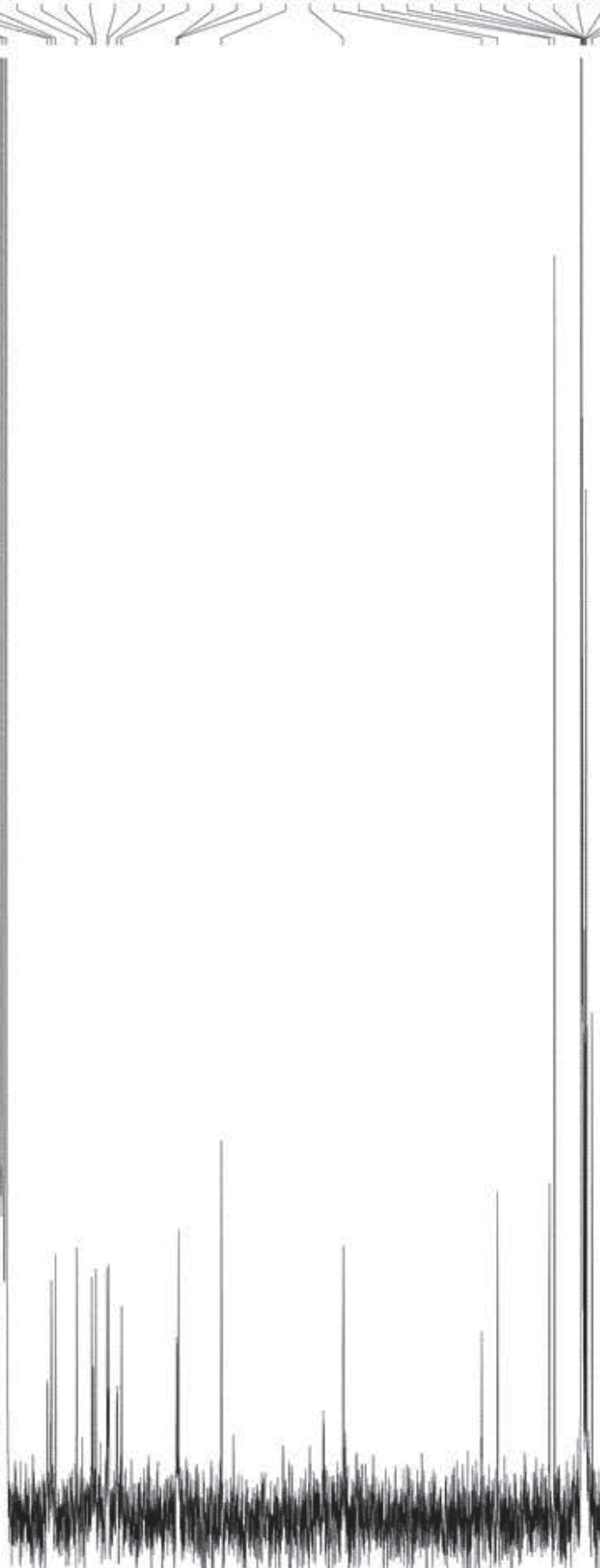

S75

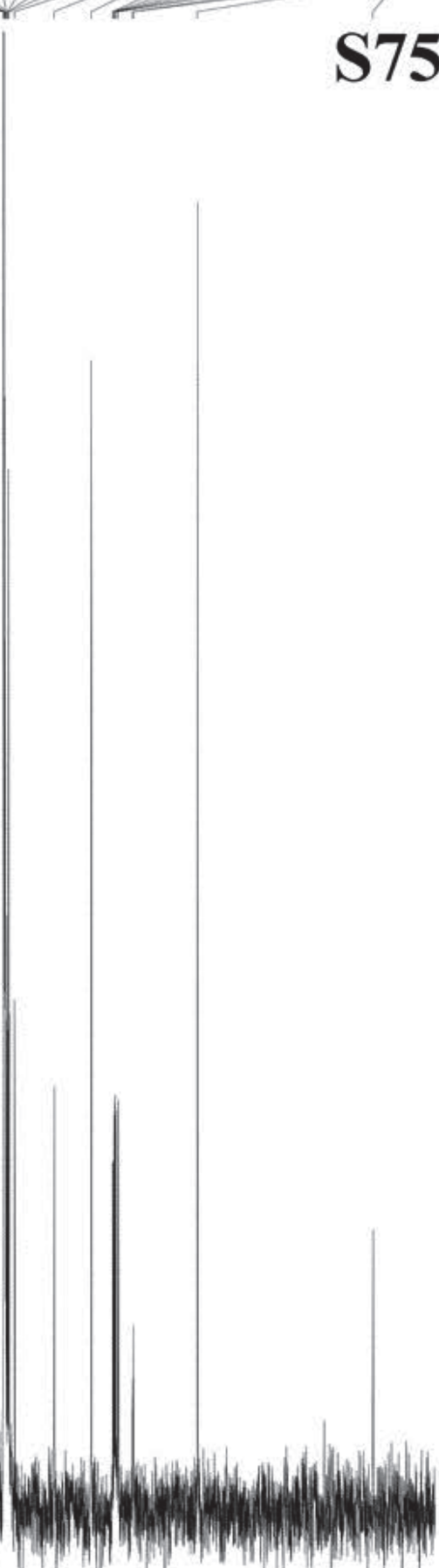




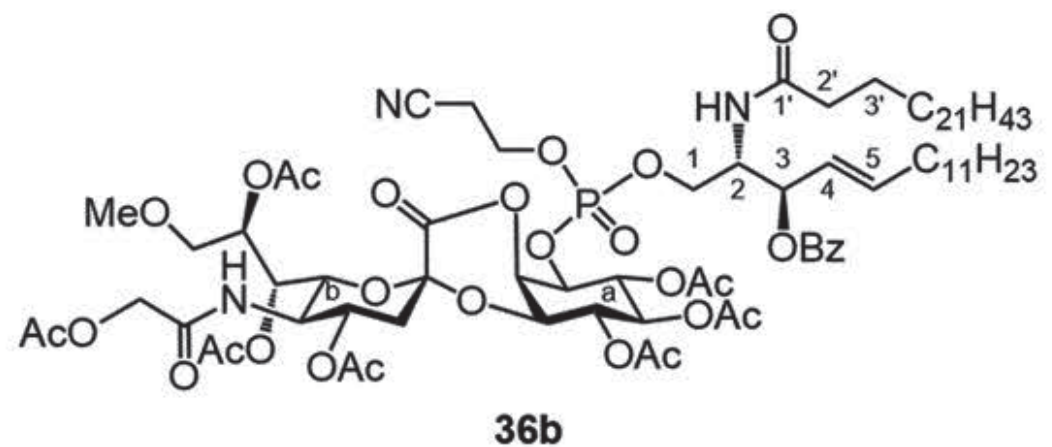

S76

${ }^{31} \mathrm{P}$ NMR (202 MHz, $\mathrm{CDCl}_{3}$ ) 


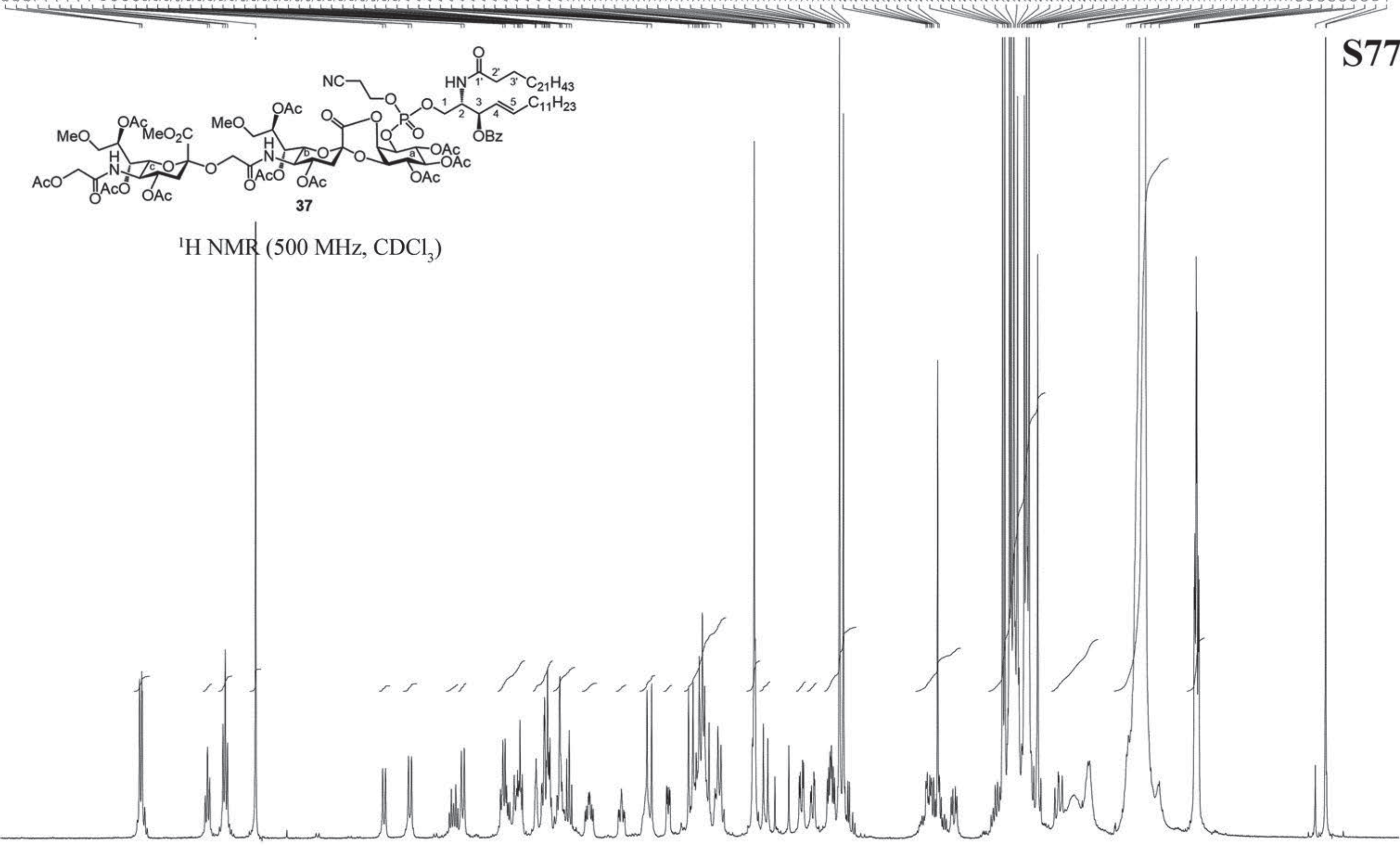




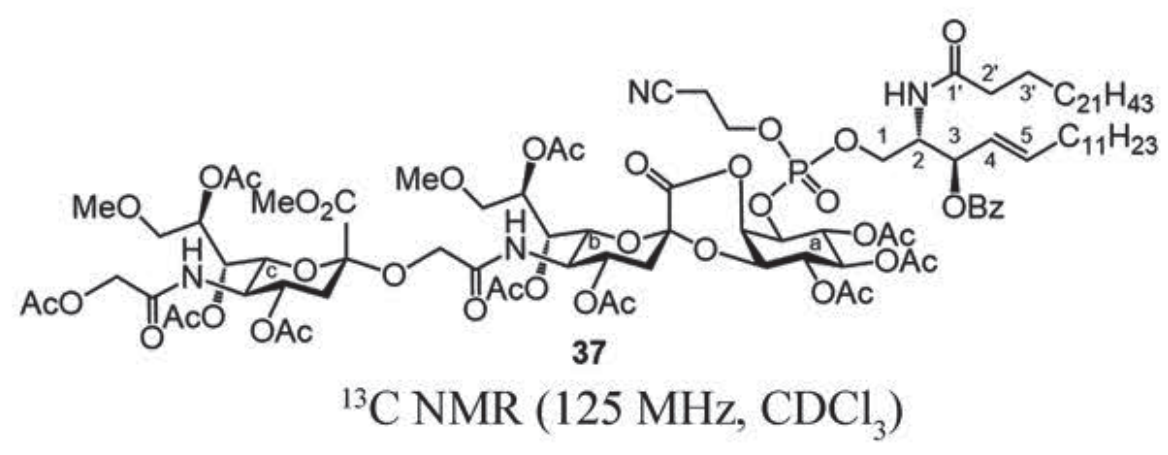

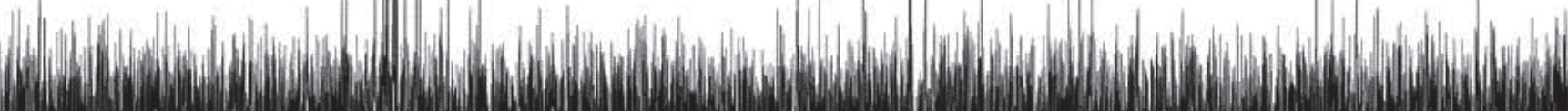

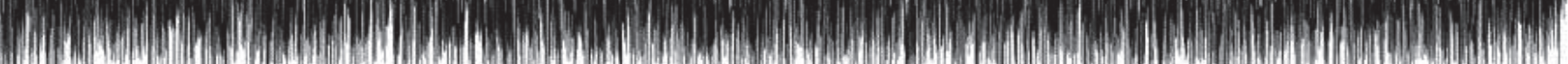

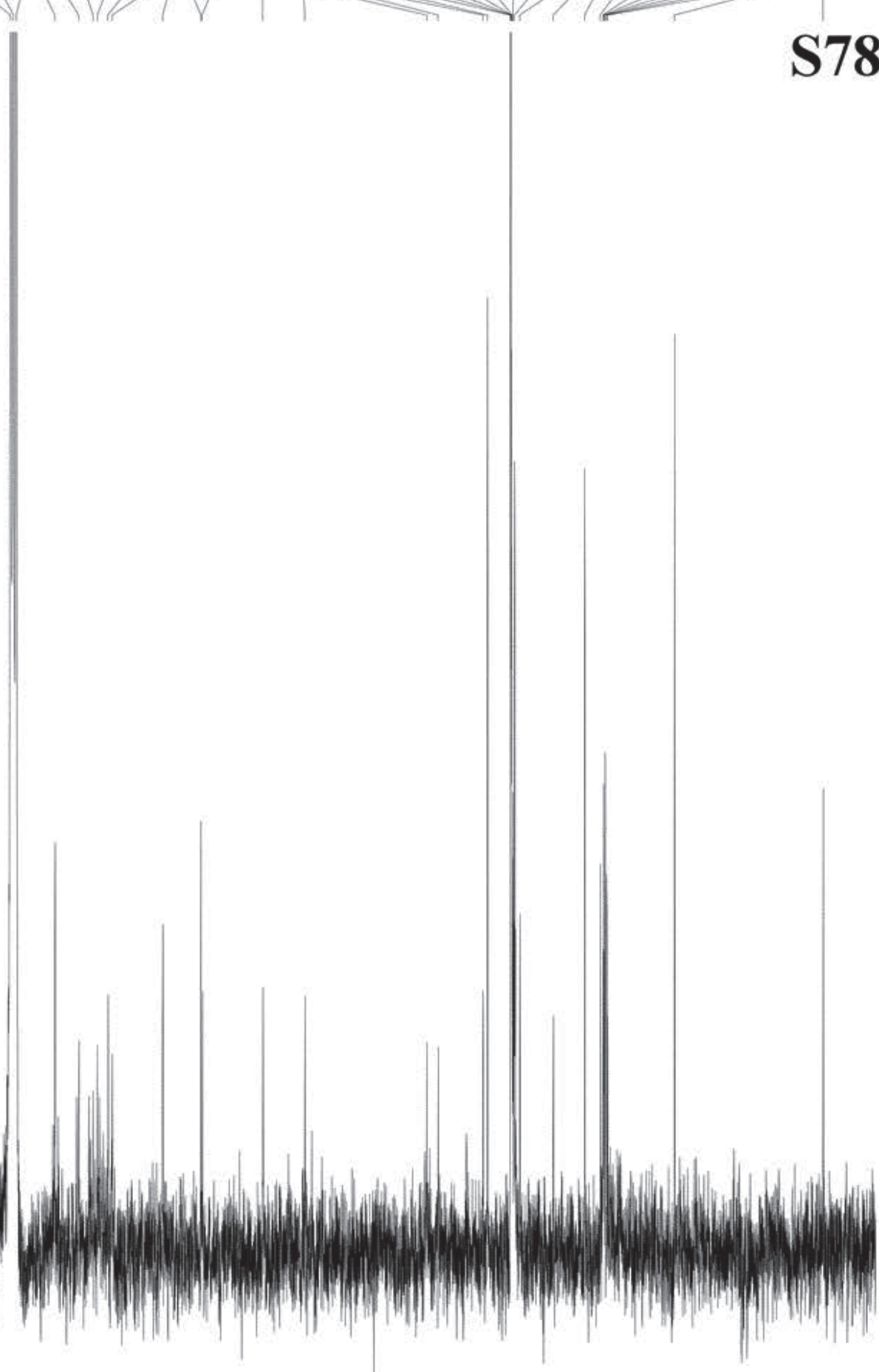




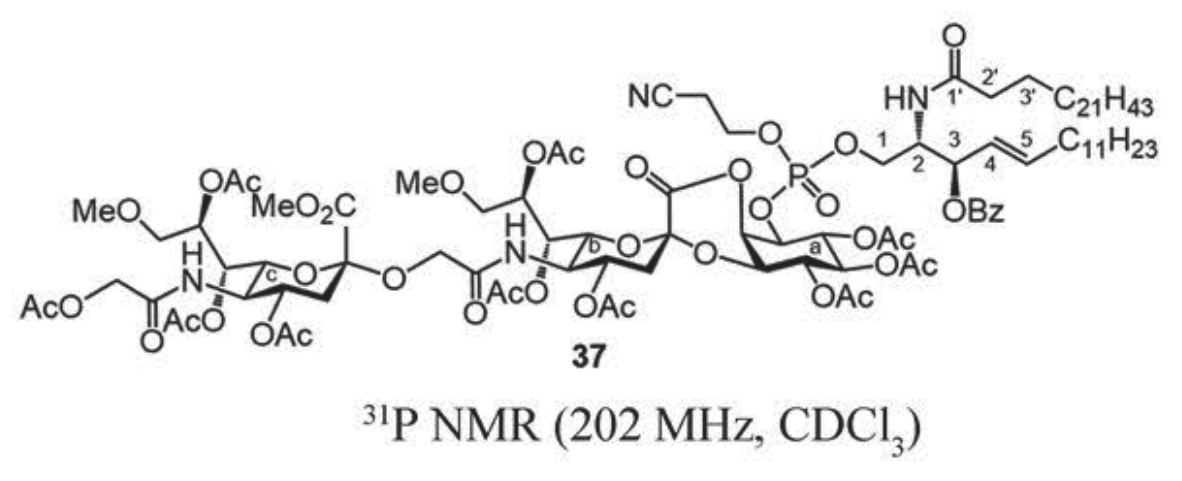

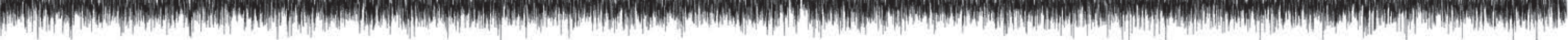




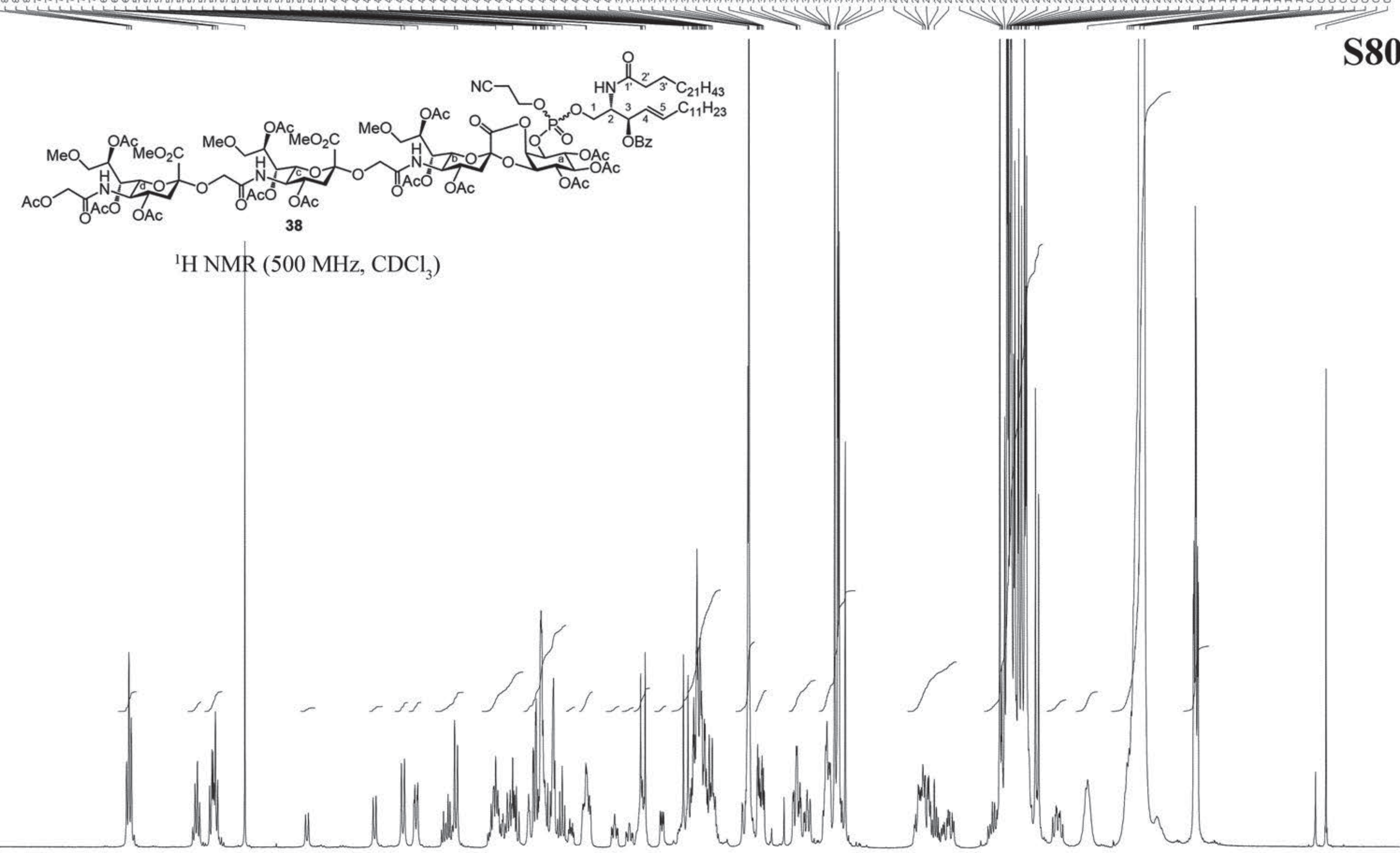




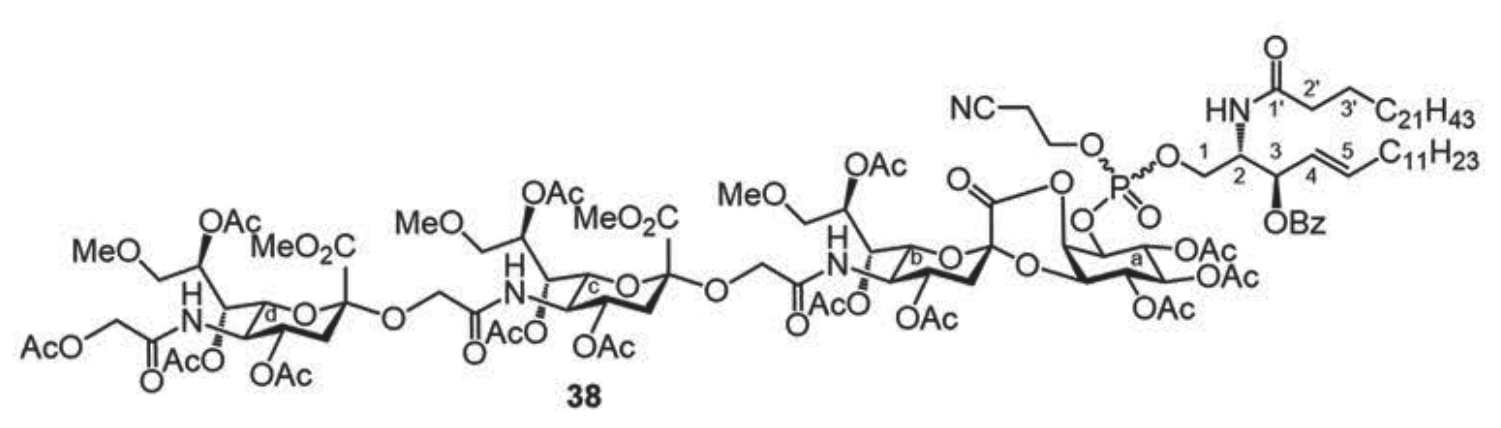

${ }^{13} \mathrm{C}$ NMR $\left(125 \mathrm{MHz}, \mathrm{CDCl}_{3}\right)$

\section{S81}

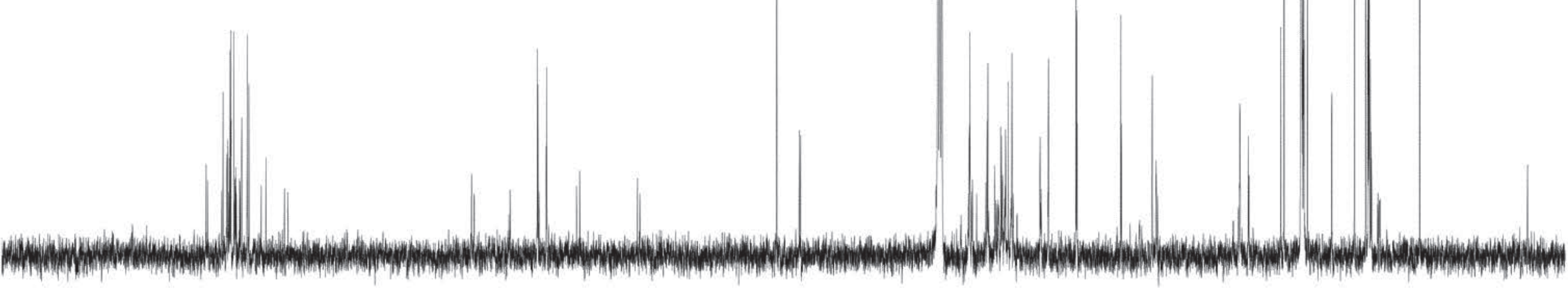




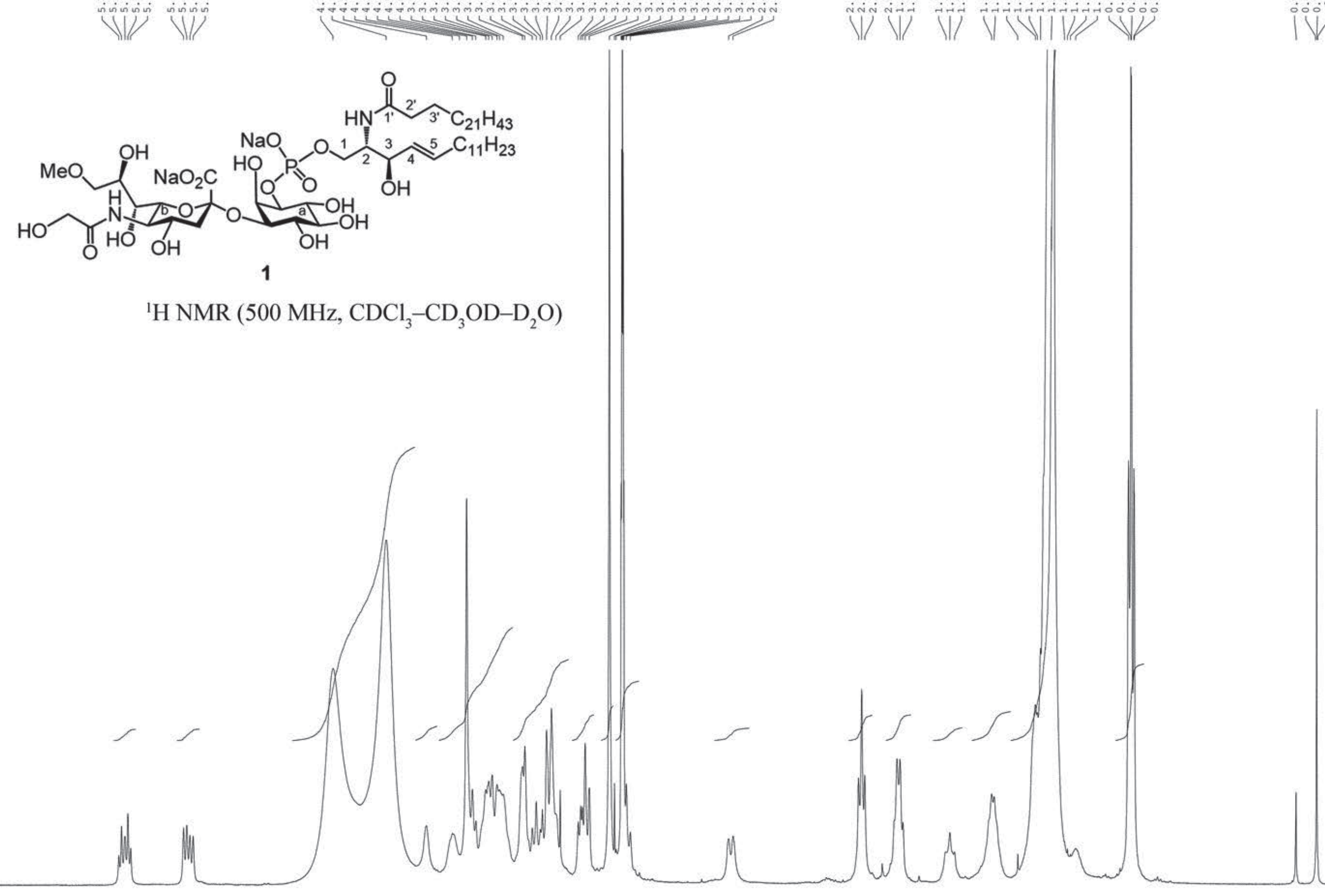

S82 


\section{S84}

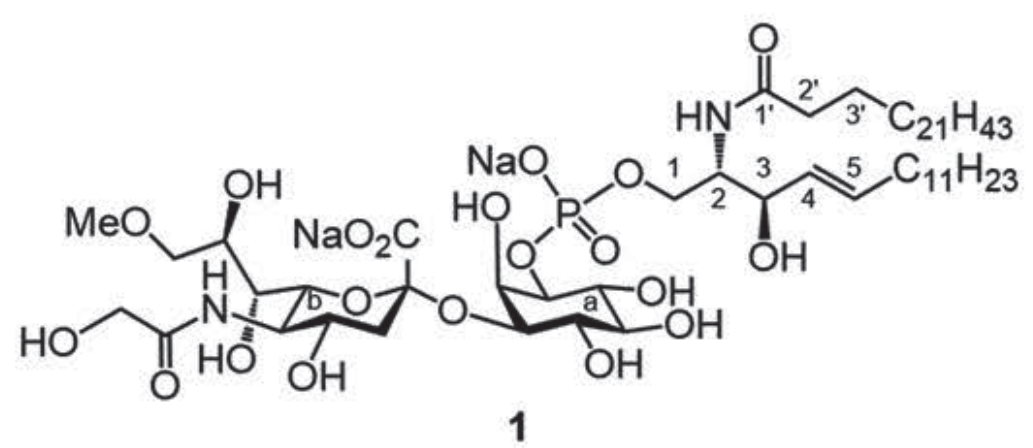

${ }^{31} \mathrm{P}$ NMR (202 MHz, $\left.\mathrm{CDCl}_{3}-\mathrm{CD}_{3} \mathrm{OD}-\mathrm{D}_{2} \mathrm{O}\right)$

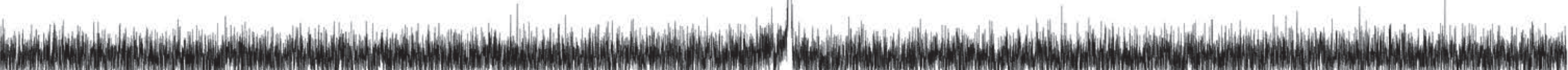
Wham 


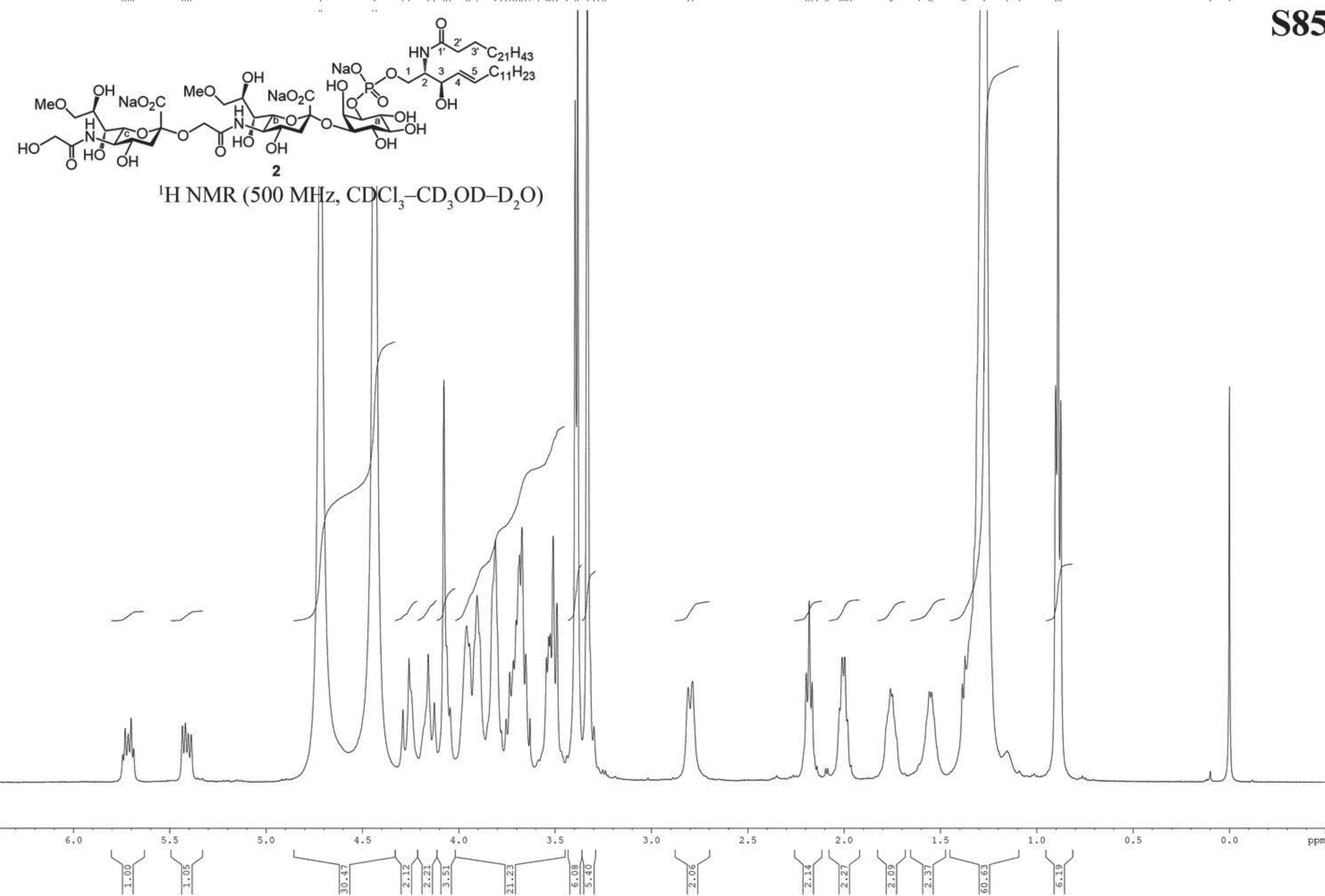




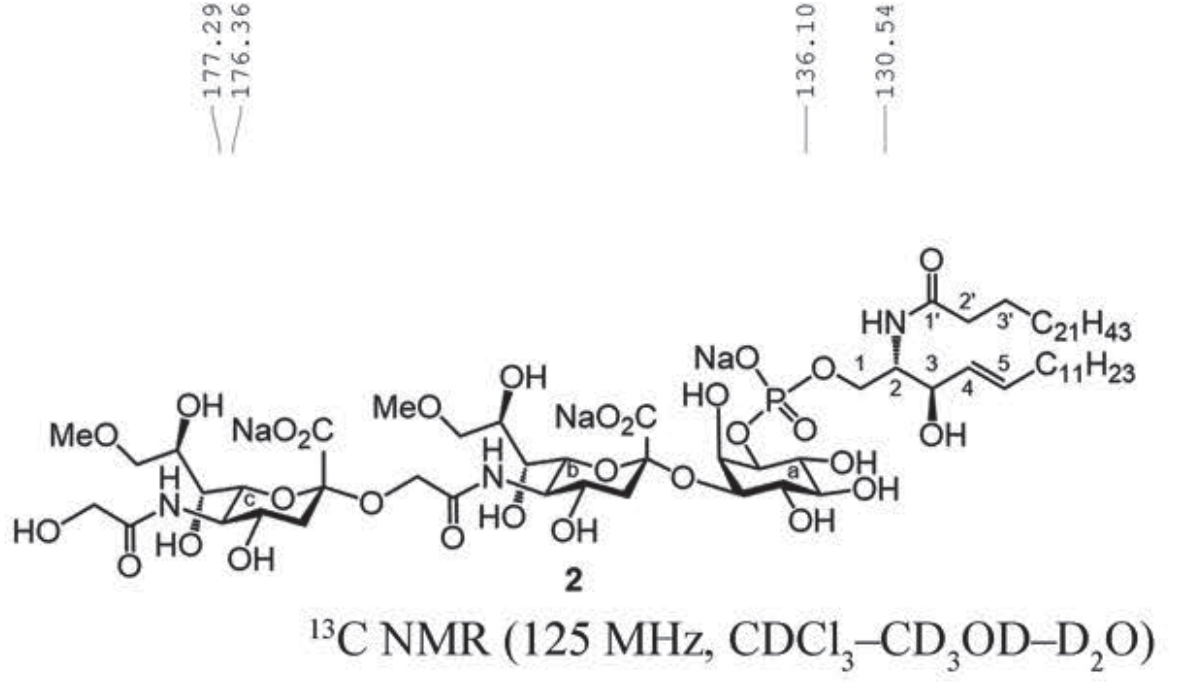




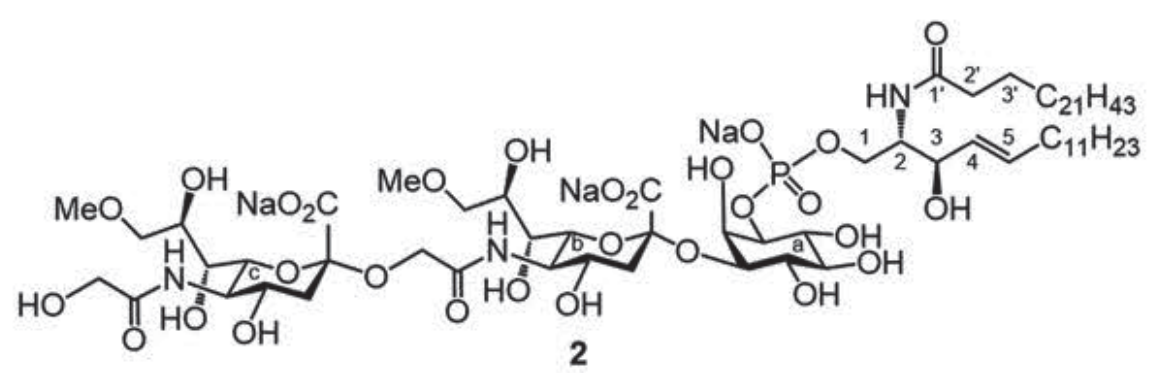

${ }^{31} \mathrm{P}$ NMR (202 MHz, $\mathrm{CDCl}_{3}-\mathrm{CD}_{3} \mathrm{OD}-\mathrm{D}_{2} \mathrm{O}$ )

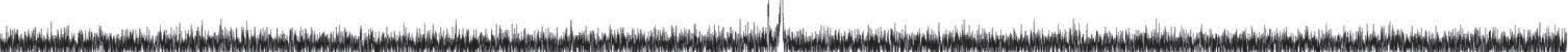
min 


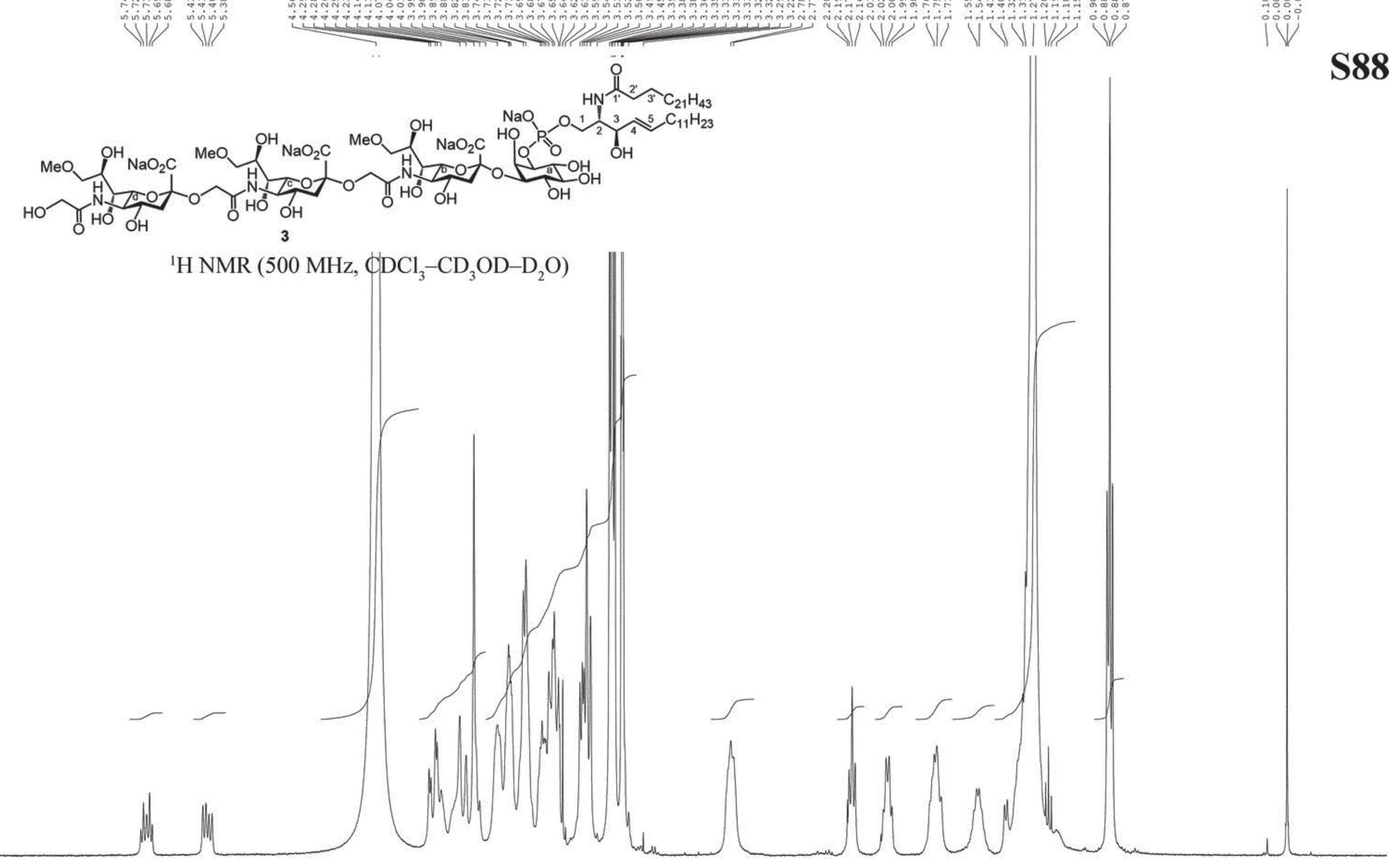




\section{S90}

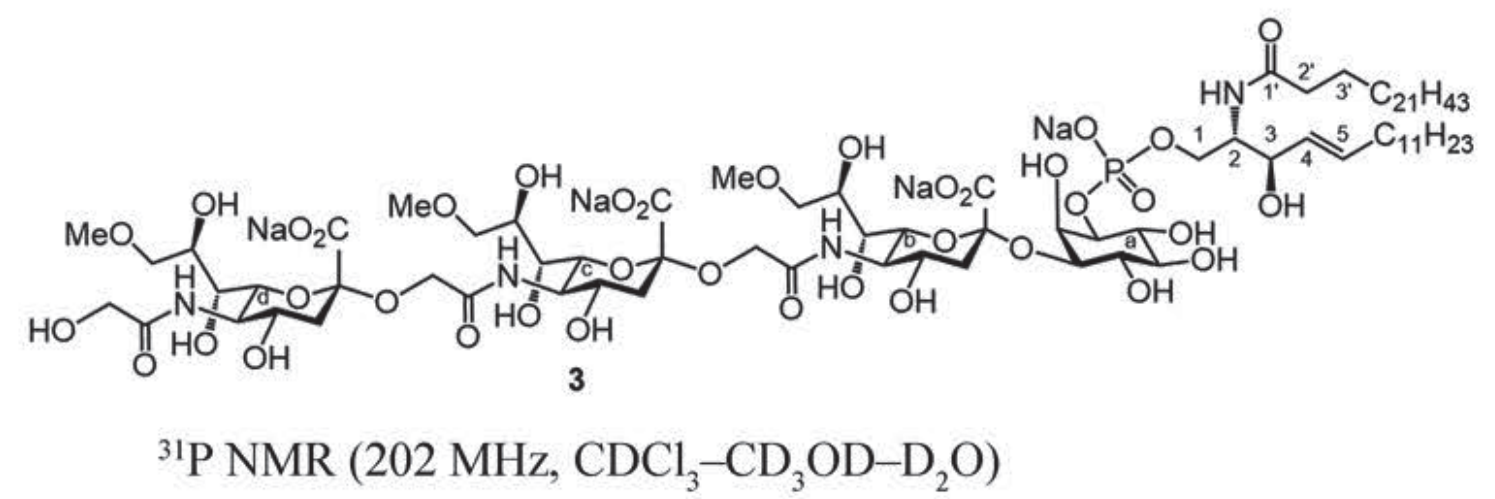

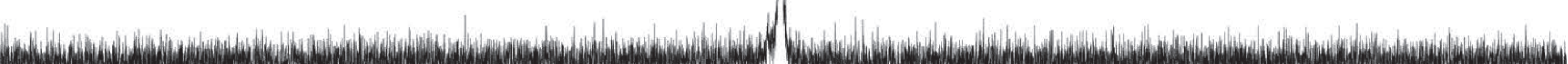
CW. 